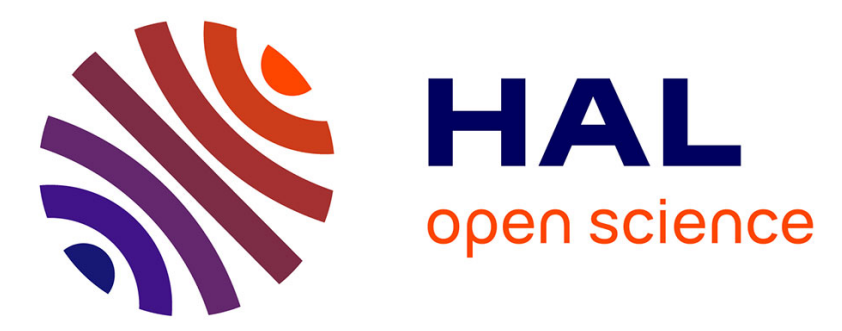

\title{
The Second-Order Coding Rate of the MIMO Quasi-Static Rayleigh Fading Channel
}

\author{
Jakob Hoydis, Romain Couillet, Pablo Piantanida
}

\section{To cite this version:}

Jakob Hoydis, Romain Couillet, Pablo Piantanida. The Second-Order Coding Rate of the MIMO Quasi-Static Rayleigh Fading Channel. IEEE Transactions on Information Theory, 2015, 61 (12), pp.6591 - 6622. 10.1109/TIT.2015.2490066 . hal-01257572

\section{HAL Id: hal-01257572 \\ https://hal.science/hal-01257572}

Submitted on 19 May 2020

HAL is a multi-disciplinary open access archive for the deposit and dissemination of scientific research documents, whether they are published or not. The documents may come from teaching and research institutions in France or abroad, or from public or private research centers.
L'archive ouverte pluridisciplinaire HAL, est destinée au dépôt et à la diffusion de documents scientifiques de niveau recherche, publiés ou non, émanant des établissements d'enseignement et de recherche français ou étrangers, des laboratoires publics ou privés. 


\title{
The Second-Order Coding Rate of the MIMO Quasi-Static Rayleigh Fading Channel
}

\author{
Jakob Hoydis, Member, IEEE, Romain Couillet, Member, IEEE, and Pablo Piantanida, Member, IEEE
}

\begin{abstract}
The second-order coding rate of the multiple-input multiple-output (MIMO) quasi-static Rayleigh fading channel is studied. We tackle this problem via an information-spectrum approach and statistical bounds based on recent random matrix theory techniques. We derive a central limit theorem (CLT) to analyze the information density in the regime where the blocklength $n$ and the number of transmit and receive antennas $K$ and $N$, respectively, grow simultaneously large. This result leads to the characterization of closed-form upper and lower bounds on the optimal average error probability when the coding rate is within $\mathcal{O}(1 / \sqrt{n K})$ of the asymptotic capacity.
\end{abstract}

Index Terms-Finite block-length, second-order coding rate, error probability, quasi-static fading channel, block-fading channel, MIMO, information spectrum, random matrix theory.

\section{INTRODUCTION}

In real-world wireless communications, the codeword (or block) length of the transmission is naturally limited due to delay and complexity constraints. It is thus unfortunate that only few tractable performance limits of wireless communication scenarios under the finite block-length regime are available. In general, only bounds on the optimal error probability for a given coding rate and block-length are derivable, e.g., [1], [2], which are for most relevant cases difficult to analyze and evaluate. This is in particular the case for non-ergodic channels (e.g., quasi-static or block-fading channels), for which the error probability is fundamentally limited by the outage probability [3]. The evaluation of these non-asymptotic bounds becomes even more challenging in presence of multiple-input multiple-output (MIMO) channels.

Feinstein [1] and Shannon [4] were among the first to explore the tradeoff between coding rate, error probability, and block-length and developed bounds on the optimal error probability in the finite block-length regime. Bounds on the limit of the scaled logarithm of the error probability-known as the

This work has been presented in part at the IEEE International Symposium on Information Theory (ISIT'12), Cambridge, MA, USA, Jul. 2012, and the IEEE International Symposium on Information Theory (ISIT'13), Istanbul, Turkey, Jul. 2013.

J. Hoydis is with Bell Laboratories, Alcatel-Lucent, Route de Villejust, 91620 Nozay, France (jakob.hoydis@alcatel-lucent.com).

R. Couillet is with Laboratoire de Signaux et Systèmes (L2S, UMR8506), CNRS-CentraleSupélec-Université Paris-Sud, 3 rue Joliot-Curie, 91192 Gifsur-Yvette, France (romain.couillet@centralesupelec.fr). Couillet's work is supported by the ERC MORE EC-120133.

P. Piantanida is with Laboratoire de Signaux et Systèmes (L2S, UMR8506), CNRS-CentraleSupélec-Université Paris-Sud, 3 rue Joliot-Curie, 91192 Gifsur-Yvette, France (pablo.piantanida@centralesupelec.fr). The work of Piantanida was partially supported by the DIM COREPHY project and the FP7 Network of Excellence in Wireless COMmunications NEWCOM\#.

Copyright (c) 2014 IEEE. Personal use of this material is permitted. However, permission to use this material for any other purposes must be obtained from the IEEE by sending a request to pubs-permissions@ieee.org. exponential rate of decrease-were derived in [2]. A simpler formula for the latter was then provided by Gallager [5], which is still difficult to evaluate for wireless channel models. In [6], an explicit expression of Gallager's error exponent was found for the block-fading MIMO channel, but the computation of this result remains quite involved.

Since the aforementioned bounds are in general not amenable to simple evaluation, asymptotic considerations were made, in particular by Strassen [7] who derived a general expression of the error probability for the discrete memoryless channel with unconstrained inputs of code length $n$ in the regime where the coding rate is within $\mathcal{O}(1 / \sqrt{n})$ of the capacity, which is referred to as the second-order coding rate. In his work, the variance of the "mutual information density" appears to be the fundamental quantity when focusing on Gaussian approximations of the error probability. Nevertheless, Strassen's approach could not be generalized to channels with input constraints, such as the additive white Gaussian noise (AWGN) channel. Hayashi [8] focused on the second-order coding rate and provided an exact characterization of the optimal error probability for different channel models and input constraints. Further considerations were made by Polyanskiy-Poor-Verdú in [9] where several novel results are provided for memoryless channels, among which new upper and lower bounds on the maximal achievable rate for a fixed error probability and block-length. Along the same lines, the scalar AWGN blockfading channel was addressed in the coherent and non-coherent settings in [10] and [11], respectively.

Additional work on the asymptotic block-length regime via information-spectrum methods comprises the general capacity formula by Verdú-Han [12] proving the converse via a novel lower bound on the error probability from [13], [14]. A very comprehensive literature survey on related aspects can also be found in [9]. During the revision of this article, we became aware of the related works [15] and [16] which study respectively the quasi-static fading single-input multipleoutput (SIMO) and MIMO channel at finite block-length in great detail.

In this paper, we investigate closed-form bounds on the average error probability of the $N \times K$ MIMO quasi-static Rayleigh fading channel where the transmission takes place over $n$ channel uses during which the channel realization is randomly drawn but remains constant, and where $N, K$, and $n$ are of similar order of magnitude.

\section{A. Contribution and outline}

We focus on the asymptotic behavior of the error probability when the coding rate is a small perturbation of the ergodic 
capacity, and hence follow the line of work of [8] on the second-order coding rate (see also [9, Section IV]). We take the approach of inducing ergodicity in the inherently nonergodic quasi-static fading channel by growing the channel matrix dimensions. Indeed, assuming an $N \times K$ channel matrix with independent standard Gaussian entries, letting $K, N \rightarrow \infty$ at the same speed, the channel becomes ergodic in the limit (even for a single channel use). This ensures that communications at rates arbitrarily close to the asymptotic capacity are possible in this regime and it becomes natural to investigate the optimal average error probability for the second-order coding rate when $K, N$, and the block-length $n$ grow simultaneously, i.e., the asymptotically achievable error probability for rates within $\mathcal{O}(1 / \sqrt{n K})$ of the ergodic capacity ( $n K$ being the total number of symbols in each codeword).

Our approach closely follows the information spectrum methodology of [8]. We first start from some basic variations of Feinstein's and Verdú-Han's lemma that provide, respectively, lower and upper bounds on the optimal error probability. These bounds are exploited to study the second-order statistics of the information density, seen as a real functional of three large-dimensional random matrices, i.e., the $N \times K$ channel, the $K \times n$ input, and the $N \times n$ noise matrices. The analysis of such statistics naturally requires the use of random matrix tools, and in particular here of Gaussian methods such as developed by Pastur [17].

The main contribution of this paper is to derive a central limit theorem (CLT) uniformly over the set of admissible channel inputs. From this result it entails that the optimal average error probability $\mathbb{P}_{e}(r \mid \beta, c)$ for the second order coding rate $r<0$ (defined in (14) below) can be bounded as

$$
\Phi\left(\frac{r}{\theta_{-}}\right) \leq \mathbb{P}_{e}(r \mid \beta, c) \leq \Phi\left(\frac{r}{\theta_{+}}\right)
$$

where $\beta=n / K, c=N / K, \Phi(\cdot)$ is the Gaussian distribution function, and $\theta_{+}>\theta_{-}$are closed-form functions of $\beta, c$, and the signal-to-noise ratio (SNR). Unlike [8], [9], we do not obtain matching lower and upper bounds due to the presence of the non-ergodic random channel matrix. Nonetheless, it appears that the gap between both bounds is quite tight for SNR values of practical interest. Besides, numerical comparisons to LDPC codes reveal good similarities with theory in the slope of the error probability.

\section{Notation and definitions}

The set of nonnegative integers is denoted by $\mathbb{N}$, the real and complex fields by $\mathbb{R}$ and $\mathbb{C}$, respectively. Boldface letters $\mathbf{X}$ and upper-case letters $\mathbf{X}$ are used to denote vectors and matrices, respectively. The transpose, complex conjugate, and complex conjugate (Hermitian) transpose are denoted by $(\cdot)^{\top}$, $(\cdot)^{*}$, and $(\cdot)^{\mathrm{H}}$, respectively. The trace and determinant of a square matrix $\mathbf{X}$ are written $\operatorname{tr} \mathbf{X}$ and $\operatorname{det}(\mathbf{X})$, respectively. The spectral norm of a square matrix $\mathbf{X}$, i.e., the absolute largest eigenvalue, is denoted by $\|\mathbf{X}\|$. The Frobenius norm of a matrix $\mathbf{X}$ is denoted by $\|\mathbf{X}\|_{F}$. The $(i, j)$-element of $\mathbf{X}$ is denoted by $\mathbf{X}_{i j}$ or $[\mathbf{X}]_{i j}$. Random vectors and matrix variables are denoted by lowercase letters $x$ and uppercase letters $X$, respectively. The symbol $\operatorname{Pr}[\cdot]$ denotes the probability of the bracketed random argument. For a set $\mathcal{S}$, we define by $\mathcal{P}(\mathcal{S})$ the set of probability measures with support a subset of $\mathcal{S}$. We also denote by $\operatorname{supp}(\mathbb{P})$ the support of $\mathbb{P}$.

For random matrices $X, Y$ in $\mathbb{C}^{K \times n}$ and $\mathbb{C}^{N \times n}$, let $\mathbb{P}_{X} \in$ $\mathcal{P}\left(\mathbb{C}^{K \times n}\right)$ and let $\mathbf{X} \mapsto \mathbb{P}_{Y \mid X}(\cdot \mid \mathbf{X})$ be any Borel measurable mapping. We define the probability measure $\mathbb{P}_{X Y}$ by $\mathbb{P}_{X Y}(\mathcal{A} \times \mathcal{B})=\int_{\mathcal{A}} \mathbb{P}_{Y \mid X}(\mathcal{B} \mid \mathbf{X}) \mathbb{P}_{X}(d \mathbf{X})$ where $\mathcal{A}, \mathcal{B}$ are Borel sets of $\mathbb{C}^{K \times n}$ and $\mathbb{C}^{N \times n}$, respectively. Similarly, we define the distribution $\mathbb{P}_{Y}$ as $\mathbb{P}_{Y}(\mathcal{B})=\int \mathbb{P}_{Y \mid X}(\mathcal{B} \mid \mathbf{X}) \mathbb{P}_{X}(d \mathbf{X})$ for any Borel subset $\mathcal{B} \subset \mathbb{C}^{N \times n}$, where the integral is understood to be taken over $\mathbb{C}^{K \times n}$. We also define, for a $\mathbb{P}_{X}$-measurable functional $f$, its mean $\mathbb{E}[f(X)]=\int f(\mathbf{X}) \mathbb{P}_{X}(d \mathbf{X})$ and variance $\operatorname{Var}[f(X)]=\mathbb{E}\left[|f(X)-\mathbb{E}[f(X)]|^{2}\right]$.

Let $\mathbb{P}$ and $\mathbb{Q}$ be two measures on (the Borel $\sigma$-field of) $\mathbb{C}^{K \times n}$. Then $\mathbb{P}$ is said to be absolutely continuous with respect to $\mathbb{Q}$ if $\mathbb{P}(\mathcal{A})=0$ for every Borel set $\mathcal{A}$ for which $\mathbb{Q}(\mathcal{A})=0$. This is written as $\mathbb{P} \ll \mathbb{Q}$. For such measures $\mathbb{P}$ and $\mathbb{Q}$, we denote $\frac{d \mathbb{P}}{d \mathbb{Q}}(\mathbf{X})=\frac{\mathbb{P}(d \mathbf{X})}{\mathbb{Q}(d \mathbf{X})}$ the Radon-Nykodym derivative [18, Theorem 32.2] of $\mathbb{P}$ with respect to $\mathbb{Q}$ at position $\mathbf{X}$, i.e., for any Borel set $\mathcal{A}, \mathbb{P}(\mathcal{A})=\int_{\mathcal{A}} \frac{d \mathbb{P}}{d \mathbb{Q}} d \mathbb{Q}=\int_{\mathcal{A}} \frac{\mathbb{P}(d \mathbf{X})}{\mathbb{Q}(d \mathbf{X})} \mathbb{Q}(d \mathbf{X})$. The notation $\mathbb{P}(d \mathbf{X}) \leq \mathbb{Q}(d \mathbf{X})$ will then be understood as $\frac{d \mathbb{P}}{d \mathbb{Q}}(\mathbf{X})=\frac{\mathbb{P}(d \mathbf{X})}{\mathbb{Q}(d \mathbf{X})} \leq 1$. If $\mathbb{P}$ is not absolutely continuous with respect to $\mathbb{Q}$, we set $d \mathbb{P} / d \mathbb{Q} \triangleq \infty$ and $\mathbb{P}(d \mathbf{X}) \leq \mathbb{Q}(d \mathbf{X})$ is understood as an always false statement.

We denote $\mathcal{C N}\left(0, \sigma^{2}\right)$ the complex circularly symmetric normal distribution with zero mean and variance $\sigma^{2}$. We call $\Phi$ the distribution function of the real standard normal distribution, given by $\Phi(x) \triangleq \frac{1}{\sqrt{2 \pi}} \int_{-\infty}^{x} \exp \left(-\frac{t^{2}}{2}\right) d t$. The weak convergence of the sequence of probability measures $\left\{\mu_{n}\right\}_{n=1}^{\infty}$ to $\mu$ is denoted by $\mu_{n} \Rightarrow \mu$; “ $\stackrel{\text { a.s. }}{\longrightarrow}$ " stands for almost sure convergence.

The notation $f_{n}(t)=\mathcal{O}\left(t^{\alpha} n^{-\beta}\right)$ means that there exists $C>0$ independent of $t$ and $n$ such that, for all $t>0$ and $n \in \mathbb{N},\left|f_{n}(t)\right| \leq C t^{\alpha} n^{-\beta}$.

\section{CHANNEL MODEL AND PROBLEM STATEMENT}

Consider the following MIMO memoryless Gaussian quasistatic fading channel:

$$
\mathbf{y}_{t}=\frac{1}{\sqrt{K}} \mathbf{H}^{n} \mathbf{x}_{t}+\sigma \mathbf{w}_{t}, \quad t=\{1, \ldots, n\}
$$

where $\mathbf{y}_{t} \in \mathbb{C}^{N}$ is the channel output at time $t, \mathbf{H}^{n} \in \mathbb{C}^{N \times K}$ is a realization of the random channel matrix $H^{n} \in \mathbb{C}^{N \times K}$ whose entries are independent and identically distributed (i.i.d.) $\mathcal{C N}(0,1)$ and the index $n$ reminds that $\mathbf{H}^{n}$ is constant for the duration of $n$ channel uses, $\mathbf{x}_{t} \in \mathbb{C}^{K \times 1}$ is the realization of the random channel input $x_{t} \in \mathbb{C}^{K \times 1}$ at time $t$, and $\sigma \mathbf{w}_{t}$ is the realization of the random noise vector $\sigma w_{t}$ at time $t$ whose entries are i.i.d. $\mathcal{C N}\left(0, \sigma^{2}\right)$. The transmitter end has only statistical knowledge about $H^{n}$ while the receiver end knows $H^{n}$ perfectly. In particular, we will assume $H^{n}$, $x_{t}$, and $w_{t}$ to be independent for each $t$. We define the following matrices: $\mathbf{X}^{n}=\left(\mathbf{x}_{1}, \ldots, \mathbf{x}_{n}\right) \in \mathbb{C}^{K \times n}, \mathbf{W}^{n}=$ $\left(\mathbf{w}_{1}, \ldots \mathbf{w}_{n}\right) \in \mathbb{C}^{N \times n}$, and $\mathbf{Y}^{n}=\left(\mathbf{y}_{1}, \ldots, \mathbf{y}_{n}\right) \in \mathbb{C}^{N \times n}$. Associated to these matrices, we define the random matrices 
$X^{n}=\left(x_{1}, \ldots, x_{n}\right) \in \mathbb{C}^{K \times n}, W^{n}=\left(w_{1}, \ldots, w_{n}\right) \in \mathbb{C}^{N \times n}$, and $Y^{n}=\left(y_{1}, \ldots, y_{n}\right) \in \mathbb{C}^{N \times n}$.

We denote the sets of admissible inputs $\mathbf{X}^{n}$ with unit maximal and exact energy constraint, respectively, by

$$
\begin{aligned}
& \mathcal{S}^{n} \triangleq\left\{\mathbf{X}^{n} \in \mathbb{C}^{K \times n} \mid \frac{1}{n K} \operatorname{tr} \mathbf{X}^{n}\left(\mathbf{X}^{n}\right)^{\mathrm{H}} \leq 1\right\} \\
& \mathcal{S}_{=}^{n} \triangleq\left\{\mathbf{X}^{n} \in \mathbb{C}^{K \times n} \mid \frac{1}{n K} \operatorname{tr} \mathbf{X}^{n}\left(\mathbf{X}^{n}\right)^{\mathbf{H}}=1\right\} .
\end{aligned}
$$

The mutual information density of $\mathbb{P}_{Y^{n} \mid X^{n}, H^{n}}$, i.e., the probability measure of $Y^{n}$ conditioned on $X^{n}$ and $H^{n}$, is defined by (see e.g. [19] for the AWGN definition):

$$
I_{N, K}^{(n)} \triangleq \frac{1}{n K} \log \frac{\mathbb{P}_{Y^{n} \mid X^{n}, H^{n}}\left(d Y^{n} \mid X^{n}, H^{n}\right)}{\mathbb{P}_{Y^{n} \mid H^{n}}\left(d Y^{n} \mid H^{n}\right)}
$$

where the ratio $\mathbb{P}_{Y^{n} \mid X^{n}, H^{n}}\left(\cdot \mid \mathbf{X}^{n}, \mathbf{H}^{n}\right) / \mathbb{P}_{Y^{n} \mid H^{n}}\left(\cdot \mid \mathbf{H}^{n}\right)$, for given $\mathbf{X}^{n}, \mathbf{H}^{n}$, denotes the Radon-Nykodym derivative of the measure $\mathbb{P}_{Y^{n} \mid X^{n}, H^{n}}\left(\cdot \mid \mathbf{X}^{n}, \mathbf{H}^{n}\right)$ with respect to $\mathbb{P}_{Y^{n} \mid H^{n}}\left(\cdot \mid \mathbf{H}^{n}\right)$ whenever $\mathbb{P}_{Y^{n} \mid X^{n}, H^{n}}\left(\cdot \mid \mathbf{X}^{n}, \mathbf{H}^{n}\right) \ll \mathbb{P}_{Y^{n} \mid H^{n}}\left(\cdot \mid \mathbf{H}^{n}\right)$ and is set to $\infty$ otherwise.

Definition 1 (Code and average error probability): A $\left(P_{e}^{(n)}, M_{n}\right)$-code $\mathcal{C}_{n}$ for the channel model (2) with power constraint (3) consists of the following mappings:

- An encoder mapping:

$$
\varphi: \mathcal{M}_{n} \longmapsto \mathbb{C}^{K \times n} .
$$

The transmitted symbols are $\mathbf{X}_{m}^{n}=\varphi(m) \in \mathcal{S}^{n}$ for every message $m$ uniformly distributed over the set $\mathcal{M}_{n}=\left\{1, \ldots, M_{n}\right\}$ of messages.

- A set of decoder mappings $\left\{\phi_{\mathbf{H}^{n}}\right\}_{\mathbf{H}^{n} \in \mathbb{C}^{N \times K}}$ with:

$$
\phi_{\mathbf{H}^{n}}: \mathbb{C}^{N \times n} \longmapsto \mathcal{M}_{n} \cup\{e\}
$$

which produces the decoder's decision $\hat{m}=\phi_{\mathbf{H}^{n}}\left(\mathbf{Y}_{m}^{n}\right)$, $\mathbf{Y}_{m}^{n}=\frac{1}{\sqrt{K}} \mathbf{H}^{n} \varphi(m)+\sigma \mathbf{W}^{n}$, on the transmitted message $m$, or the error event $e$.

For a code $\mathcal{C}_{n}$ with block-length $n$, codebook size $M_{n}$, encoder $\varphi$, and decoder $\left\{\phi_{\mathbf{H}^{n}}\right\}_{\mathbf{H}^{n} \in \mathbb{C}^{N \times K}}$, the average error probability is defined as

$$
P_{e}^{(n)}=P_{e}^{(n)}\left(\mathcal{C}_{n}\right) \triangleq \operatorname{Pr}[\hat{m} \neq m],
$$

where the probability is taken over the random variables $W^{n}$, $H^{n}$ and $m$.

Let $\operatorname{supp}\left(\mathcal{C}_{n}\right)$ denote the codebook $\left\{\varphi(1), \ldots, \varphi\left(M_{n}\right)\right\}$. The optimal average error probability for the rate $R$ is defined as

$$
\mathbb{P}_{e}^{(n)}(R) \triangleq \inf _{\mathcal{C}_{n}: \operatorname{supp}\left(\mathcal{C}_{n}\right) \subseteq \mathcal{S}^{n}}\left\{P_{e}^{(n)}\left(\mathcal{C}_{n}\right) \mid \frac{1}{n K} \log M_{n} \geq R\right\} .
$$

The exact characterization of $\mathbb{P}_{e}^{(n)}(R)$ for fixed $n, K$, and $N$ is generally intractable. As mentioned in the introduction, a classical approach consists in considering rates within $\mathcal{O}(1 / \sqrt{n})$ of the ergodic capacity with block-lengths $n$ growing to infinity (i.e., second-order coding rates). This leads to tractable limiting error probabilities, referred to as optimal average error probabilities for the second-order coding rates [8], [9]. However, as the capacity of the quasi-static Rayleigh fading channel is zero, we assume here that the system dimensions $K$ and $N$ grow large. This induces ergodicity in the channel and entails a new definition of the second-order coding rate and the optimal average error probability for the quasi-static fading MIMO channel. Precisely, we assume that $K, N$, and $n$ are large but of the same order of magnitude. This is expressed mathematically via the relations

$$
n \rightarrow \infty, \quad \frac{n}{K}=\beta, \quad \frac{N}{K}=c
$$

for some constants $\beta, c>011$ These relations will be denoted by $n \stackrel{(\beta, c)}{\longrightarrow} \infty$ in the remainder of the article. For an infinite block-length, the per-antenna capacity of the channel converges for almost every channel realization to an asymptotic limit $C$ [20]:

Theorem 1 ([20 Eq. (9)],[21. Thm. 1]): Let $\left\{H^{n}\right\}_{n=1}^{\infty}$, where $H^{n} \in \mathbb{C}^{N \times K}$ has i.i.d. entries $H_{i j}^{n} \sim \mathcal{C N}(0,1)$. Let $\sigma^{2}>0$ and define

$$
C_{N, K} \triangleq \frac{1}{K} \log \operatorname{det}\left(\mathbf{I}_{N}+\frac{1}{\sigma^{2} K} H^{n}\left(H^{n}\right)^{\mathrm{H}}\right) .
$$

Then, as $n \stackrel{(\beta, c)}{\longrightarrow} \infty$,

(i) $C_{N, K} \stackrel{\text { a.s. }}{\longrightarrow} C\left(\sigma^{2}\right)$

(ii) $\mathbb{E}\left[C_{N, K}\right]=C\left(\sigma^{2}\right)+\mathcal{O}\left(\frac{1}{n^{2}}\right)$

where, for $x>0$,

$$
\begin{aligned}
C(x)= & \log \left(1+\delta_{0}(x)\right)+c \log \left(1+\frac{1}{x\left(1+\delta_{0}(x)\right)}\right) \\
& -\frac{\delta_{0}(x)}{1+\delta_{0}(x)}
\end{aligned}
$$

and

$$
\delta_{0}(x) \triangleq \frac{c-1}{2 x}-\frac{1}{2}+\frac{\sqrt{(1-c+x)^{2}+4 c x}}{2 x}>0 .
$$

Based on this observation, we can characterize the error probability in the second-order coding rate, i.e., when the coding rate is within $\mathcal{O}(1 / \sqrt{n K})$ of the limiting capacity $C=C\left(\sigma^{2}\right)$, and estimate $\mathbb{P}_{e}^{(n)}(R)$ via the following limiting error probability:

Definition 2: The optimal average error probability for the second-order coding rate $r$ is

$$
\begin{gathered}
\mathbb{P}_{e}(r \mid \beta, c) \triangleq \inf _{\left\{\mathcal{C}_{n}: \operatorname{supp}\left(\mathcal{C}_{n}\right) \subseteq \mathcal{S}^{n}\right\}_{n=1}^{\infty}}\left\{\limsup _{n \stackrel{(\beta, c)}{\longrightarrow} \infty} P_{e}^{(n)}\left(\mathcal{C}_{n}\right) \mid\right. \\
\left.\liminf _{n \stackrel{(\beta, c)}{\longrightarrow} \infty} \sqrt{n K}\left(\frac{1}{n K} \log M_{n}-C\right) \geq r\right\} .
\end{gathered}
$$

Remark 1 (Fluctuation around ergodic capacity): For the channel model (2), the optimal average error probability may

\footnotetext{
${ }^{1}$ This assumption can be relaxed to $\frac{n}{K}=\beta+o\left(n^{-2}\right)$ and $\frac{N}{K}=c+$ $o\left(n^{-2}\right)$. However, it is easy to see that these constraints impose $c$ and $\beta$ to be rational numbers and the sequences $\{N / K\}_{n=1}^{\infty}$ and $\{n / K\}_{n=1}^{\infty}$ to be constant for all large $n$.
} 
be alternatively written as

$$
\begin{aligned}
& \mathbb{P}_{e}(r \mid \beta, c)= \inf _{\substack{\left\{\mathcal{C}_{n}\right\}_{n=1}^{\infty} \\
\operatorname{supp}\left(\mathcal{C}_{n}\right) \subseteq \mathcal{S}^{n}}}\left\{\limsup _{n \stackrel{(\beta, c)}{\longrightarrow} \infty} P_{e}^{(n)}\left(\mathcal{C}_{n}\right) \mid\right. \\
&\left.\liminf _{n \stackrel{(\beta, c)}{\longrightarrow} \infty} \sqrt{n K}\left(\frac{1}{n K} \log M_{n}-\mathbb{E}\left[C_{N, K}\right]\right) \geq r\right\}
\end{aligned}
$$

since

$$
\sqrt{n K}\left(\mathbb{E}\left[C_{N, K}\right]-C\right) \rightarrow 0
$$

as $n \stackrel{(\beta, c)}{\longrightarrow} \infty$ by Theorem 1 (ii). In the finite $N, K, n$-regime, we may therefore see the optimal average error probability as an approximation of the optimal achievable error under the rate constraint

$$
\frac{1}{n K} \log M_{n} \geq \mathbb{E}\left[C_{N, K}\right]+\frac{r}{\sqrt{n K}} .
$$

Note that the relation (16) is fundamentally dependent on the Gaussianity of $H^{n}$. It was indeed shown in [22, Theorem 4.4] that, whenever the entries of $H^{n}$ have a non-zero fourth order cumulant $\kappa=\mathbb{E}\left[\left|H_{11}^{n}\right|^{4}\right]-2$, a bias term $\mathcal{B}$ proportional to $\kappa$ arises such that (16) must be modified to $\sqrt{n K}\left(\mathbb{E}\left[C_{N, K}\right]-C\right) \rightarrow \mathcal{B}$ as $n \stackrel{(\beta, c)}{\longrightarrow} \infty$. In this case the equivalence of (15) and (14) does not hold. For Gaussian channels (since $\kappa=0$ and then $\mathcal{B}=0$ ), however, the asymptotic mutual information is reached at the sufficiently fast rate of $\mathcal{O}\left(n^{-2}\right)$ (as confirmed by Theorem $1(i i)$ ).

Instead of the optimal average error probability, we may consider the second-order outage probability $\mathbb{P}_{\text {out }}(r \mid \beta, c)$ for the rate $r$, which we define as follows:

Definition 3: The second-order outage probability for the second-order coding rate $r$ is

$$
\begin{gathered}
\mathbb{P}_{\text {out }}(r \mid \beta, c) \triangleq \inf _{\left\{\mathcal{C}_{n}: \operatorname{supp}\left(\mathcal{C}_{n}\right) \subseteq \mathcal{S}^{n}\right\}_{n=1}^{\infty}}\left\{\limsup _{n \stackrel{(\beta, c)}{\longrightarrow} \infty} P_{e}^{(n)}\left(\mathcal{C}_{n}\right) \mid\right. \\
\left.\liminf _{n \stackrel{(\beta, c)}{\longrightarrow} \infty} K\left(\frac{1}{n K} \log M_{n}-C\right) \geq r\right\}
\end{gathered}
$$

The second-order outage probability and the optimal average error probability are related by $\mathbb{P}_{\text {out }}(r \mid \beta, c)=\mathbb{P}_{e}(r \sqrt{\beta} \mid \beta, c)$. Definition 3 allows us to study the behavior of the secondorder outage probability for growing $\beta$. In the finite dimensional setting, this corresponds to increasing the block-length while maintaining $N$ and $K$ (and thus the capacity $K C$ ) fixed. This cannot be performed on $\mathbb{P}_{e}(r \mid \beta, c)$ since, by growing $n$, $\sqrt{n K} C$ grows as well, therefore not maintaining the capacity fixed as $n$ grows alone.

The main objective of this article is to characterize $\mathbb{P}_{e}(r \mid \beta, c)$ (which will in turn characterize $\mathbb{P}_{\text {out }}(r \mid \beta, c)$ ).

\section{MAIN RESULT}

To determine the optimal average error probability, one ideally needs to determine the asymptotic fluctuations of the mutual information density $I_{N, K}^{(n)}$ for all codes $\mathcal{C}_{n}$. Since this is intractable, we shall resort to upper and lower bounds, which shall both rely on establishing the fluctuations of the random quantity $I_{N, K}^{X^{n}}$ as defined, for $\mathbb{P}_{X^{n}} \in \mathcal{P}\left(\mathcal{S}^{n}\right)$, in (19) on the top of the next page.

These fluctuations are provided in the following theorem.

Theorem 2: Let $\left\{X^{n}\right\}_{n=1}^{\infty}$ be a sequence of random variables with probability $\mathbb{P}_{X^{n}} \in \mathcal{P}\left(\mathcal{S}_{=}^{n}\right)$ and, for $A^{n}=\mathbf{I}_{K}-$ $\frac{1}{n} X^{n}\left(X^{n}\right)^{\mathrm{H}}$, define $\theta_{n}>0$ the random variable given by

$$
\begin{gathered}
\theta_{n}^{2}=-\beta \log \left(1-\frac{1}{c} \frac{\delta_{0}\left(\sigma^{2}\right)^{2}}{\left(1+\delta_{0}\left(\sigma^{2}\right)\right)^{2}}\right)+c+\sigma^{4} \delta_{0}^{\prime}\left(\sigma^{2}\right) \\
-\beta \frac{\delta_{0}^{\prime}\left(\sigma^{2}\right)}{\left(1+\delta_{0}\left(\sigma^{2}\right)\right)^{4}} \frac{1}{K} \operatorname{tr}\left[\left(A^{n}\right)^{2}\right]
\end{gathered}
$$

where the function $\delta_{0}(x)$ is defined in (13). Then, for any real $z$, as $n \stackrel{(\beta, c)}{\longrightarrow} \infty$,

$$
\operatorname{Pr}\left[\frac{\sqrt{n K}}{\theta_{n}}\left(I_{N, K}^{X^{n}}-C\right) \leq z\right] \rightarrow \Phi(z) .
$$

Proof: The proof is provided in Appendix D-B

Based on this result, we can determine the following lower and upper bounds on the optimal average error probability for the second-order coding rate.

Theorem 3: The optimal average error probability $\mathbb{P}_{e}(r \mid \beta, c)$ for the second-order coding rate $r$ satisfies:

- If $r \leq 0$,

$$
\Phi\left(\frac{r}{\theta_{-}}\right) \leq \mathbb{P}_{e}(r \mid \beta, c) \leq \Phi\left(\frac{r}{\theta_{+}}\right)
$$

- If $r>0$,

$$
\frac{1}{2} \leq \mathbb{P}_{e}(r \mid \beta, c) \leq \Phi\left(\frac{r}{\theta_{+}}\right)
$$

where $\theta_{-}>0$ and $\theta_{+}>0$ are defined by

$$
\begin{aligned}
\theta_{-}^{2} \triangleq & -\beta \log \left(1-\frac{1}{c} \frac{\delta_{0}\left(\sigma^{2}\right)^{2}}{\left(1+\delta_{0}\left(\sigma^{2}\right)\right)^{2}}\right) \\
& +c+\sigma^{4} \delta_{0}^{\prime}\left(\sigma^{2}\right) \\
\theta_{+}^{2} \triangleq- & \beta \log \left(1-\frac{1}{c} \frac{\delta_{0}\left(\sigma^{2}\right)^{2}}{\left(1+\delta_{0}\left(\sigma^{2}\right)\right)^{2}}\right) \\
& +c+\sigma^{4} \delta_{0}^{\prime}\left(\sigma^{2}\right)-\frac{\delta_{0}^{\prime}\left(\sigma^{2}\right)}{\left(1+\delta_{0}\left(\sigma^{2}\right)\right)^{4}}
\end{aligned}
$$

and $\delta_{0}(x)$ is defined in (13) with derivative, for $x>0$,

$$
\delta_{0}^{\prime}(x)=-\frac{\delta_{0}(x)\left(1+\delta_{0}(x)\right)}{1-c+x+2 x \delta_{0}(x)}<0 .
$$

Proof: The details of this proof are provided in Appendix B

Theorem 3 shows that, for sufficiently large channel dimensions and block-length, the optimal error probability for a coding rate close to the asymptotic capacity, i.e., $(n K)^{-1} \log M_{n}=C+(n K)^{-1 / 2} r$, is comprised between two explicit bounds which depend only on $c, \beta$, and $\sigma^{2}$. This is to be compared with the AWGN scenario of [8], [9] where the corresponding bounds were found to depend only on $\sigma^{2}$. However, as opposed to Theorem 3, the lower and upper bounds in these works were shown to be equal. We discuss in Remark 3 below the technical reasons for this important 


$$
\begin{aligned}
I_{N, K}^{X^{n}} \triangleq & \frac{1}{K} \log \operatorname{det}\left(\mathbf{I}_{N}+\frac{1}{\sigma^{2}} \frac{H^{n}\left(H^{n}\right)^{\mathrm{H}}}{K}\right) \\
& +\frac{1}{n K} \operatorname{tr}\left[\left(\frac{H^{n}\left(H^{n}\right)^{\mathrm{H}}}{K}+\sigma^{2} \mathbf{I}_{N}\right)^{-1}\left(\frac{H^{n}}{\sqrt{K}} X^{n}+\sigma W^{n}\right)\left(\frac{H^{n}}{\sqrt{K}} X^{n}+\sigma W^{n}\right)^{\mathrm{H}}-W^{n}\left(W^{n}\right)^{\mathrm{H}}\right]
\end{aligned}
$$

difference. Note that, for rates above the capacity limit (i.e., for $r>0$ ), the lower bound is very loose and can be far from its associated upper bound. In contrast, the more interesting case $r<0$ (corresponding to coding rates below the asymptotic capacity) features two bounds which are numerically shown to be quite close to one-another.

Remark 2 (On the quantity $\delta_{0}\left(\sigma^{2}\right)$ ): The function $c^{-1} \delta_{0}\left(\sigma^{2}\right)$ coincides with the Stieltjes transform $m_{\mu_{c}}(z)$ of the Marčenko-Pastur measure $\mu_{c}$ with parameter $c$ [23] evaluated at position $z=-\sigma^{2}$, which is defined by $m_{\mu_{c}}(z)=\int(t-z)^{-1} \mu_{c}(d t)$ for all $z \in \mathbb{C} \backslash \operatorname{supp}\left(\mu_{c}\right)$. This measure is the limiting distribution of the eigenvalues of $K^{-1} H^{n}\left(H^{n}\right)^{\mathrm{H}}$ as $N, K \rightarrow \infty$ and $N / K \rightarrow c$. For this reason, the quantities $C, \theta_{-}$, and $\theta_{+}$of Theorem 3 naturally appear as functionals of $\mu_{c}$.

Remark 3 (Tightness of the bounds): The case $r=0$ set aside, the lower and upper bounds on the optimal average error probability are never equal. This unfolds from the presence of the random channel $H^{n}$ which induces a dependence of the second order statistics of $I_{N, K}^{(n)}$ on the "fourth order moment" $\mathbb{E}\left[K^{-1} \operatorname{tr}\left(n^{-1} X^{n}\left(X^{n}\right)^{\mathrm{H}}\right)^{2}\right]$ of $\mathbb{P}_{X^{n}}$. The weak lower bound $1 / 2$ for $r>0$ is a consequence of the impossibility in the proof to bound the fourth order moment of $\mathbb{P}_{X^{n}}$ from above under the sole constraint (3); see Appendix B By contrast, in [8], [9], only (scalar) second order moments of $\mathbb{P}_{X^{n}}$ play a role in the second order statistics of $I_{N, K}^{(n)}$. These are easily controlled by (3).

Remark 4 (High SNR-regime): In the high-SNR regime, we have the following result:

$$
\begin{aligned}
& \lim _{\sigma^{2} \rightarrow 0} \theta_{-}^{2}= \begin{cases}-\beta \log (1-c)+c & , c<1 \\
\infty & , c=1 \\
-\beta \log \left(1-\frac{1}{c}\right)+1 & , c>1,\end{cases} \\
& \lim _{\sigma^{2} \rightarrow 0} \theta_{+}^{2}= \begin{cases}-\beta \log (1-c)+c(2-c) & , c<1 \\
\infty & , c=1 \\
-\beta \log \left(1-\frac{1}{c}\right)+1 & , c>1 .\end{cases}
\end{aligned}
$$

This follows from the definition of $\delta_{0}(x)$ and $\delta_{0}^{\prime}(x)$ in Theorem 11 which brings, for $c<1, \delta_{0}(x) \rightarrow c(1-c)^{-1}$ and $\delta_{0}^{\prime}\left(\sigma^{2}\right) \rightarrow-c(1-c)^{3}$ as $x \downarrow 0$, while, for $c>1$, $x \delta_{0}(x) \rightarrow c-1$ and $x^{2} \delta_{0}^{\prime}(x) \rightarrow 1-c$ as $x \downarrow 0$.

Remark 5 (Low SNR-regime): Both $\theta_{+}^{2}$ and $\theta_{-}^{2}$ converge to 0 as $\sigma^{2} \rightarrow \infty$. Thus, for $r<0$, the upper and lower bounds on $\mathbb{P}_{e}(r \mid \beta, c)$ are equal to zero and, for $r>0$, the upper bound tends to one. However, also the asymptotic capacity $C$ is zero. First order approximations of $C$ and $\theta_{-}^{2}, \theta_{+}^{2}$ for $\sigma^{2} \rightarrow \infty$ are thus meaningful and are given by

$$
\begin{aligned}
C & =\frac{c}{\sigma^{2}}+\mathcal{O}\left(\sigma^{-4}\right) \\
\theta_{+}^{2} & =\frac{2 c}{\sigma^{2}}+\mathcal{O}\left(\sigma^{-4}\right) \\
\theta_{-}^{2} & =\frac{2 c}{\sigma^{2}}+\mathcal{O}\left(\sigma^{-4}\right) .
\end{aligned}
$$

This shows in particular that $\left(\theta_{+}^{2}-\theta_{-}^{2}\right) / \theta_{+}^{2}=\mathcal{O}\left(\sigma^{-2}\right)$, implying the asymptotic closeness of the upper and lower bounds in the low SNR regime. Note additionally that, for $c=1$, the approximate standard deviation $\frac{2 c}{\sigma^{2}}$ coincides with the low-SNR channel dispersion reported in [9] for SISO AWGN channels.

Figure 1 depicts the bounds on the optimal average error probability for varying second-order coding rates $r$ and for different SNR values (defined as SNR $=\sigma^{-2}$ ). We choose $c=2$ and $\beta=16$. For fair comparison between the various SNR regimes, $r$ is taken to be proportional to $C\left(\sigma^{2}\right)$. For finite but large $N, K, n$ values, Figure 1 therefore provides approximate error probability bounds when coding at rate $R=$ $C\left(\sigma^{2}\right)\left(1+r^{\prime} / \sqrt{n K}\right)$ for various values of $r^{\prime}$. We observe that, for negative second-order coding rates, the gap between the upper- and lower-bound is barely visible.

Remark 6 (Relation to second-order outage probability): Recalling Definition 3 , we have

$$
\min \left\{\Phi\left(\frac{r}{\theta_{-}^{\text {out }}}\right), \frac{1}{2}\right\} \leq \mathbb{P}_{\text {out }}(r \mid \beta, c) \leq \Phi\left(\frac{r}{\theta_{+}^{\text {out }}}\right)
$$

where $\theta_{-}^{\text {out }}>0$ and $\theta_{+}^{\text {out }}>0$ are defined by

$$
\begin{aligned}
\left(\theta_{-}^{\text {out }}\right)^{2} \triangleq- & \log \left(1-\frac{1}{c} \frac{\delta_{0}\left(\sigma^{2}\right)^{2}}{\left(1+\delta_{0}\left(\sigma^{2}\right)\right)^{2}}\right) \\
& +\frac{1}{\beta}\left(c+\sigma^{4} \delta_{0}^{\prime}\left(\sigma^{2}\right)\right) \\
\left(\theta_{+}^{\text {out }}\right)^{2} \triangleq- & \log \left(1-\frac{1}{c} \frac{\delta_{0}\left(\sigma^{2}\right)^{2}}{\left(1+\delta_{0}\left(\sigma^{2}\right)\right)^{2}}\right) \\
+ & \frac{2}{\beta}\left(c+\sigma^{4} \delta_{0}^{\prime}\left(\sigma^{2}\right)-\frac{\delta_{0}^{\prime}\left(\sigma^{2}\right)}{\left(1+\delta_{0}\left(\sigma^{2}\right)\right)^{4}}\right) .
\end{aligned}
$$

Interestingly, for $r \leq 0$, as $\beta \rightarrow \infty$, we recover the limiting outage probability of MIMO Gaussian fading channels [24], [22],

$$
\lim _{\beta \rightarrow \infty} \mathbb{P}_{\text {out }}(r \mid \beta, c)=\Phi\left(\frac{r}{\theta^{\text {out }}}\right)
$$

with $\theta^{\text {out }}>0$ defined by

$$
\left(\theta^{\text {out }}\right)^{2} \triangleq-\log \left(1-\frac{1}{c} \frac{\delta_{0}\left(\sigma^{2}\right)^{2}}{\left(1+\delta_{0}\left(\sigma^{2}\right)\right)^{2}}\right) .
$$




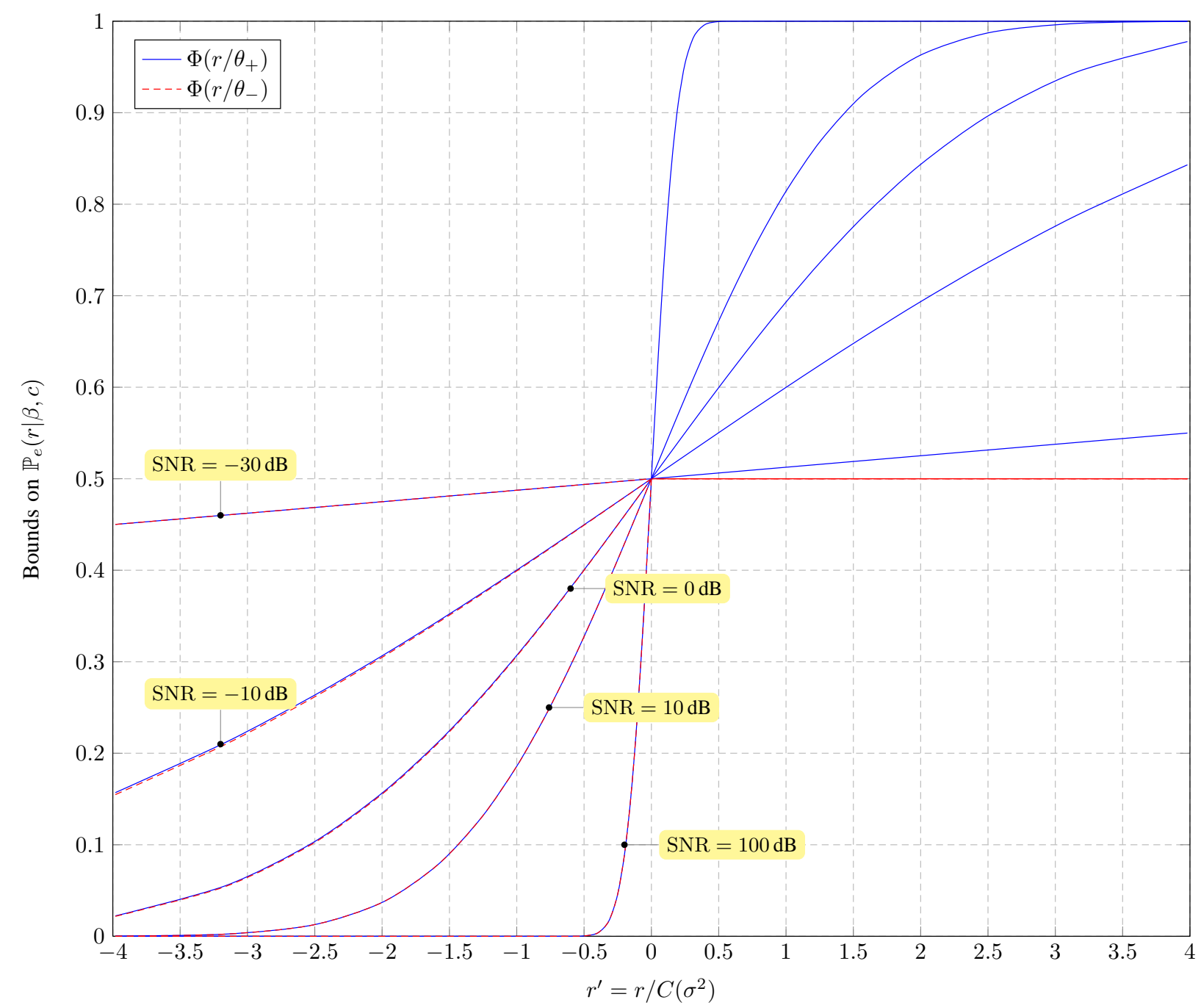

Fig. 1. Bounds on the optimal average error probability as a function of the second-order coding rate $r=r^{\prime} C\left(\sigma^{2}\right)$ for different SNRs and the parameters $c=2$ and $\beta=16$.

Although both results coincide, there is a fundamental difference in the way they are obtained. In [24], [22], the blocklength is assumed to be infinitely large from the start and then the limit is taken in $N$ and $K$. By contrast, we have obtained (35) by changing the order of both limits. Note also that, while $\Phi\left(r / \theta_{-}^{\text {out }}\right)$ and $\Phi\left(r / \theta_{+}^{\text {out }}\right)$ are decreasing functions of $\beta$ for $r<0, \Phi\left(r / \theta_{+}^{\text {out }}\right)$ is increasing in $\beta$ for $r>0$. Although no tight lower bound was derived for $r>0$, this strongly suggests the existence of a crossing point for the optimal average error probability for an error rate of $1 / 2$. We will see a practical example of this crossing point effect in Figure 3

Figure 2 depicts the bounds on $\mathbb{P}_{\text {out }}(r \mid \beta, c)$ in (32) as a function of $\beta$ for different values of $c$, assuming SNR $=10 \mathrm{~dB}$ and $r=-C\left(\sigma^{2}\right)$ (for fair comparison since $C\left(\sigma^{2}\right)$ is implicitly a function of $c$ ). For each value of $c$ we also provide the limiting outage probability as given in (35). The upper and lower bounds are seen to approach the outage probability at a rate $\mathcal{O}\left(\beta^{-1}\right)$ as $\beta$ grows, which is easily confirmed by direct calculus.

We conclude this section by a comparison in Figure 3 of the theoretical results against practical codes. We specifically consider a scenario with $K=8$ transmit and $N=16$ receive antennas employing QPSK modulation at each antenna. Coding and modulation are set up in a conventional bit-interleaved coded modulation (BICM) scheme, with a random interleaver separating the code and the modulation. At the receiver, we employ a non-iterative demodulation scheme, with a MAP MIMO demodulator based on a full code book enumeration. We consider short LDPC codes and take as an example the rate $1 / 2$ code used in the WiMAX standard [25], corresponding to a coding rate in nats $R=\log (2)$. This code is a quasicyclic irregular repeat-accumulate (IRA) LDPC code where the accumulator is slightly modified to ease the encoding circuit.

We consider code blocks of $n^{\prime}=576$ bit and $n^{\prime}=2304$ bit, corresponding to $n=n^{\prime} /(2 K) \in\{36,144\}$ channel uses. The error probability of the code described above for $n \in\{36,144\}$ is compared against the approximate upper and lower bounds (Theorem 3 ) obtained when coding at second order rate $r=\left(R-C\left(\sigma^{2}\right)\right) \sqrt{n K}$, for different SNR (i.e., $\sigma^{-2}$ ) values (corresponding to a span from $r \simeq 5.2$ for $-4 \mathrm{~dB}$ 


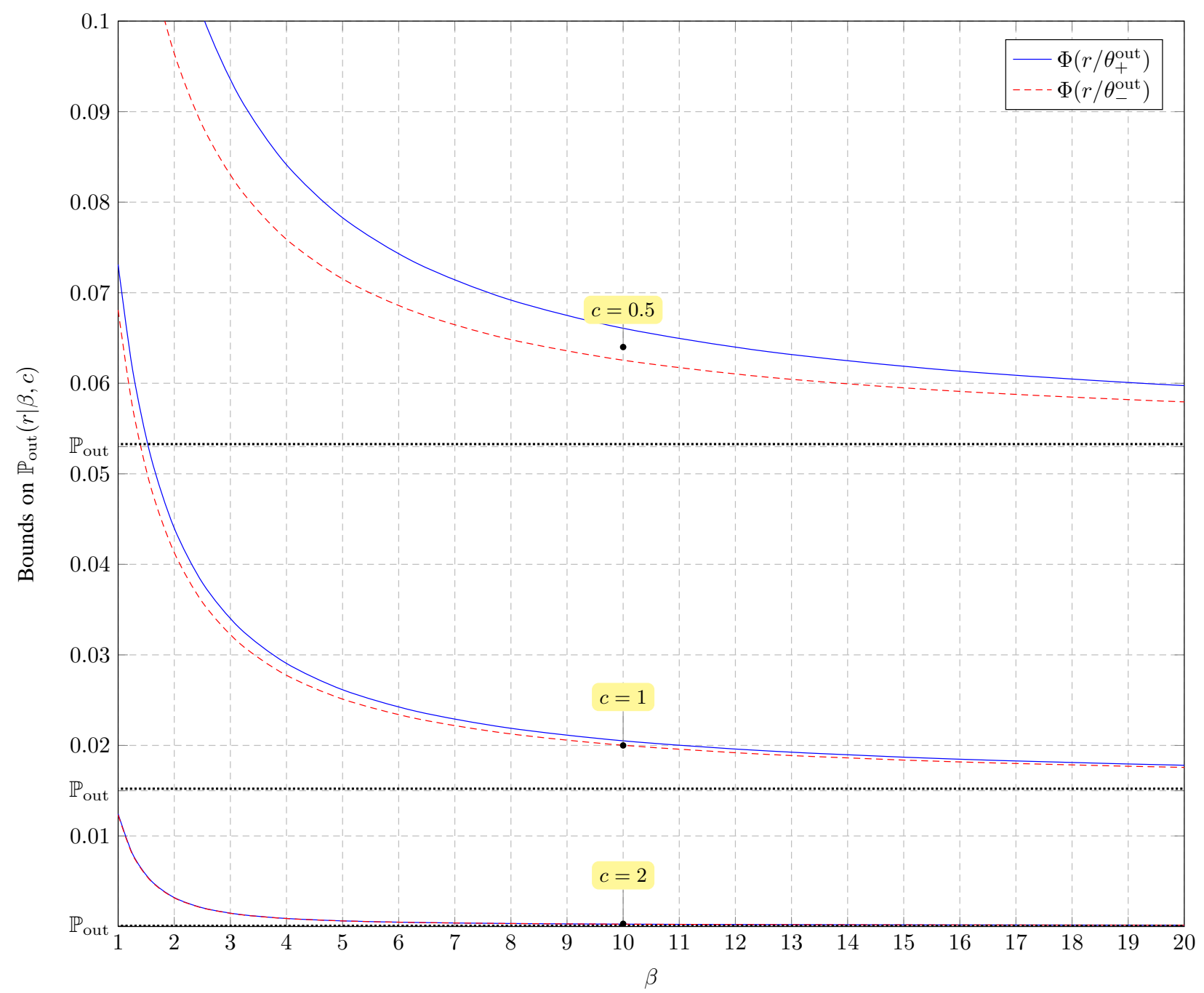

Fig. 2. Bounds on the second-order outage probability as a function of $\beta$ for different values of $c, r=-C\left(\sigma^{2}\right)$, and SNR $=10 \mathrm{~dB}$. The limiting outage probability is $\mathbb{P}_{\text {out }} \triangleq \mathbb{P}_{\text {out }}(r \mid \infty, c)$.

SNR to $r \simeq-10$ for $0 \mathrm{~dB}$ SNR, when $n=144)$. We can make several interesting observations from this figure. For both block-lengths, the SNR-gap between the simulation results and the corresponding bounds by Theorem 3 is roughly constant (to about $4 \mathrm{~dB}$ ) for a large range of SNR values.

Also note that both theoretical and simulated curves exhibit a crossing point close to $1 / 2$ error probability, which goes in line with Remark 6

\section{SUMMARY AND DIRECTIONS FOR FUTURE WORK}

We have studied the second-order coding rate of the MIMO quasi-static Rayleigh fading channel using informationspectrum methods and Gaussian tools from random matrix theory. To this end, we derived a CLT for the asymptotic analysis of the "information density" where the channel dimensions as well as the block-length grow infinitely large at the same speed and the coding rate is a perturbation within $\mathcal{O}(1 / \sqrt{n K})$ of the asymptotic capacity. The derived CLT allowed us to characterize closed-form upper and lower bounds on the optimal average error probability which depend only on the main system and channel parameters. The proposed approach to the study of the asymptotic statistics of the "mutual information density" for MIMO channels is original and can be further applied to other scenarios, such as the block-fading regime where coding is performed over multiple coherence blocks or, in a more practical context, the error performance achieved under linear receive filters.

\section{APPENDIX A}

\section{AUXILIARY RESULTS ON INFORMATION SPECTRUM}

The objective of this section is to prove Proposition 1 below which provides analytical bounds on the optimal average error probability $\mathbb{P}_{e}(r \mid \beta, c)$ and constitutes the first step of the proof of Theorem 3, developed in Appendix B.

We first state a variation of Verdú-Han's lemma [12] which appears to be more adequate to characterize the second-order approximation of the error probability.

Lemma 1 (Variation on Verdú-Han's lemma): For any integer $n \geq 1$, let $X^{n}$ be an arbitrary random variable uniformly distributed over the set of $M_{n}$ messages issued from $M_{n}$ 


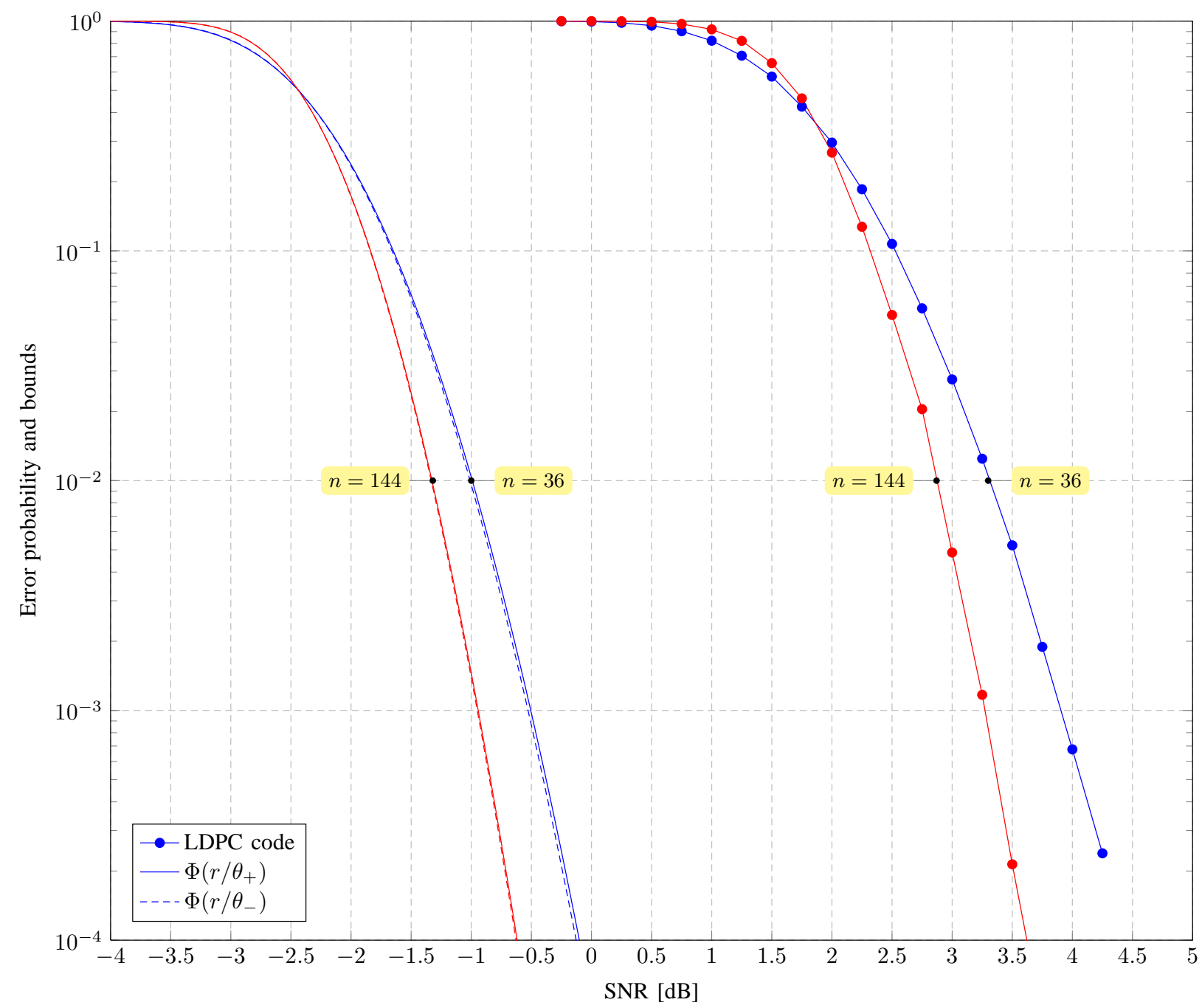

Fig. 3. Approximate bounds on the error probability for finite $n$, as a function of the SNR $=1 / \sigma^{2}, r=K(R-C)$ for $K=8, N=16, R=\log (2)$, $n \in\{36,144\}, C$ being evaluated with $c=N / K, \beta=n / K$ and for different SNR values. Theoretical curves are compared to a rate $1 / 2$ LDPC QPSK code (giving $R=\log (2)$ ).

realizations of $\mathbb{P}_{X^{n}} \in \mathcal{P}\left(\mathcal{S}^{n}\right)$, and let $Y^{n}$ be the output random variable of the channel $\mathbb{P}_{Y^{n} \mid X^{n}, H^{n}}$ corresponding to the input $X^{n}$ and the random fading $H^{n}$. Then, the average error probability of such a $\left(P_{e}^{(n)}, M_{n}\right)$-code $\mathcal{C}_{n}$ must satisfy

$$
\begin{aligned}
& P_{e}^{(n)}\left(\mathcal{C}_{n}\right) \geq \sup _{\gamma>0} \sup _{\left\{\mathbb{Q}_{n}\right\}_{n=1}^{\infty}} \\
& \left\{\operatorname{Pr}\left[\log \frac{\mathbb{P}_{Y^{n} \mid X^{n}, H^{n}}\left(d Y^{n} \mid X^{n}, H^{n}\right)}{\mathbb{Q}_{n}\left(d Y^{n} \mid H^{n}\right)} \leq \log \gamma\right]-\frac{\gamma}{M_{n}}\right\}
\end{aligned}
$$

where $\mathbb{Q}_{n}\left(\cdot \mid H^{n}\right)$ is an $H^{n}$-measurable random variable valued in $\mathcal{P}\left(\mathbb{C}^{N \times n}\right)$.

Proof: The proof follows straightforwardly from that in [12] which itself is related to [13]. We remark that a similar result was already used in [8] without an explicit proof and also follows from the same steps used to prove the "metaconverse" theorem in [9. Thm. 26, 27].

Lemma 2 (Variation of Feinstein's lemma): Let $n \geq 1$ be an integer and denote by $Y^{n}$ the output from the channel $\mathbb{P}_{Y^{n} \mid X^{n}, H^{n}}$ corresponding to an input distribution $\mathbb{P}_{X^{n}}$ and random fading $H^{n}$. We denote $\mathbb{P}_{n}\left(d Y^{n} \mid H^{n}\right)$ the distribution of such $Y^{n}$ given $H^{n}$. Then, there exists a block-length $n$ codebook of size $M_{n}$ that, together with the maximum $a$ posteriori (MAP) decoder, forms a code $\mathcal{C}_{n}$ whose average error probability $P_{e}^{(n)}\left(\mathcal{C}_{n}\right)$ satisfies:

$$
\begin{aligned}
& P_{e}^{(n)}\left(\mathcal{C}_{n}\right) \leq \\
& \inf _{\gamma>0}\left\{\operatorname{Pr}\left[\log \frac{\mathbb{P}_{Y^{n} \mid X^{n}, H^{n}}\left(d Y^{n} \mid X^{n}, H^{n}\right)}{\widetilde{\mathbb{P}}_{n}\left(d Y^{n} \mid H^{n}\right)} \leq \log \gamma \kappa\right]+\frac{M_{n}}{\gamma}\right\} \\
& +\operatorname{Pr}\left(\frac{\mathbb{P}_{n}\left(d Y^{n} \mid H^{n}\right)}{\widetilde{\mathbb{P}}_{n}\left(d Y^{n} \mid H^{n}\right)}>\kappa\right)
\end{aligned}
$$

for any probability measure $\widetilde{\mathbb{P}}_{n}\left(d Y^{n} \mid H^{n}\right) \gg \mathbb{P}_{n}\left(d Y^{n} \mid H^{n}\right)$ and positive value $\kappa$, where $\frac{\mathbb{P}_{n}\left(d Y^{n} \mid H^{n}\right)}{\mathbb{P}_{n}\left(d Y^{n} \mid H^{n}\right)}$ denotes the RadonNikodym derivative.

Proof: The proof simply follows from Feinstein's lemma [1] and the introduction of the event

$$
\mathcal{B}_{n}=\left\{Y^{n} \in \mathbb{C}^{N \times n}: \frac{\mathbb{P}_{n}\left(Y^{n} \mid H^{n}\right)}{\widetilde{\mathbb{P}}_{n}\left(Y^{n} \mid H^{n}\right)}>\kappa\right\} .
$$


In order to obtain Part-(ii) (Upper bound) of Proposition 1. below, we need the following technical result.

Lemma 3 (A divergence result): Let $\widetilde{\mathbb{P}}_{n}\left(d \widetilde{Y}^{n} \mid \mathbf{H}^{n}\right)$ and $\mathbb{P}_{n}\left(d Y^{n} \mid \mathbf{H}^{n}\right)$ be the output distributions of the channels $\widetilde{Y}^{n}=\frac{1}{\sqrt{K}} \mathbf{H}^{n} \widetilde{X}^{n}+\sigma W^{n}$ and $Y^{n}=\frac{1}{\sqrt{K}} \mathbf{H}^{n} X^{n}+\sigma W^{n}$, respectively, where $\widetilde{X}^{n}$ is standard Gaussian (i.e., with independent $\mathcal{C N}(0,1)$ entries) and $X^{n}=\sqrt{n K} \widetilde{X}^{n} /\left\|\widetilde{X}^{n}\right\|_{F}$. Then, for any sequence $\kappa_{n}$ satisfying $\kappa_{n} \rightarrow \infty$,

$$
\alpha_{n} \triangleq \operatorname{Pr}\left(\log \frac{\mathbb{P}_{n}\left(d Y^{n} \mid H^{n}\right)}{\widetilde{\mathbb{P}}_{n}\left(d Y^{n} \mid H^{n}\right)} \geq \log \kappa_{n}\right) \rightarrow 0
$$

where $\operatorname{Pr}(\cdot)$ is taken over $H^{n}$ standard Gaussian and $Y^{n} \sim \mathbb{P}_{n}$.

Proof: For two distributions $P$ and $Q$, let $\beta_{\alpha}(P, Q)$ be defined as in [9. Eq. (100)]. Then, we have the following bounds on $\beta_{\alpha}(P, Q)$ [9. Eqs. (154)-(157)]:

$$
\beta_{\alpha}(P, Q) \geq \exp \left(-\frac{D(P \| Q)+h(\alpha)}{\alpha}\right)
$$

where $D(P \| Q)$ is the Kullback-Leibler divergence and $h(x)$ the binary entropy function, and [9. Eq. (103)]

$$
\beta_{\alpha}(P, Q) \leq \frac{1}{\gamma_{0}}
$$

for any $\gamma_{0}$ satisfying

$$
\operatorname{Pr}\left(\frac{d P}{d Q} \geq \gamma_{0}\right) \geq \alpha .
$$

Setting $P=\mathbb{P}_{n}\left(d Y^{n} \mid \mathbf{H}^{n}\right), Q=\widetilde{\mathbb{P}}_{n}\left(d Y^{n} \mid \mathbf{H}^{n}\right), \alpha=\alpha_{n}$, $\gamma_{0}=\kappa_{n}$ and using the upper and lower bounds on $\beta_{\alpha}$, we conclude that

$$
\begin{aligned}
& \exp \left(-\frac{D\left(\mathbb{P}_{n}\left(d Y^{n} \mid H^{n}\right) \| \widetilde{\mathbb{P}}_{n}\left(d Y^{n} \mid H^{n}\right)\right)+h\left(\alpha_{n}\right)}{\alpha_{n}}\right) \\
& \leq \frac{1}{\kappa_{n}} \rightarrow 0 .
\end{aligned}
$$

To obtain (40), it is thus sufficient to prove $D\left(\mathbb{P}_{n}\left(d Y^{n} \mid H^{n}\right) \| \widetilde{\mathbb{P}}_{n}\left(d Y^{n} \mid H^{n}\right)\right)=\mathcal{O}(1)$.

By the data-processing inequality for the Kullback-Leibler divergence [26],

$$
\begin{aligned}
& D\left(\mathbb{P}_{n}\left(d Y^{n} \mid H^{n}\right) \| \widetilde{\mathbb{P}}_{n}\left(d Y^{n} \mid H^{n}\right)\right) \\
& =D\left(\mathbb{P}_{Y^{n} \mid H^{n}} \| \mathbb{P}_{\widetilde{Y}^{n} \mid H^{n}}\right) \\
& \leq D\left(\mathbb{P}_{Y^{n} \widetilde{X}^{n} \mid H^{n}} \| \mathbb{P}_{\widetilde{Y}^{n} \widetilde{X}^{n} \mid H^{n}}\right) \\
& =D\left(\mathbb{P}_{Y^{n} \mid H^{n} \widetilde{X}^{n}} \| \mathbb{P}_{\widetilde{Y}^{n} \mid H^{n} \widetilde{X}^{n}}\right) \\
& =\mathbb{E}_{H^{n}, \widetilde{X}^{n}}\left[D \left(\mathbb{P}_{Y^{n} \mid \mathbf{H}^{n}=H^{n}, \widetilde{\mathbf{X}}^{n}=\widetilde{X}^{n}} \| \mathbb{P}_{\left.\left.\widetilde{Y}^{n} \mid \mathbf{H}^{n}=H^{n}, \widetilde{\mathbf{X}}^{n}=\widetilde{X}^{n}\right)\right]}\right.\right.
\end{aligned}
$$

Note that, for given $\mathbf{H}^{n}, \widetilde{\mathbf{X}}^{n}$, the channel outputs $Y^{n}, \widetilde{Y}^{n}$ are Gaussian distributed, i.e.,

$$
\begin{aligned}
& \mathbb{P}_{Y^{n} \mid \mathbf{H}^{n}, \widetilde{\mathbf{X}}^{n}} \sim \mathcal{C N}\left(\operatorname{vec}\left(\frac{1}{\sqrt{K}} \mathbf{H}^{n} \frac{\sqrt{n K} \widetilde{\mathbf{X}}^{n}}{\left\|\widetilde{\mathbf{X}}^{n}\right\|_{F}}\right), \sigma^{2} \mathbf{I}_{N \times n}\right) \\
& \mathbb{P}_{\widetilde{Y}^{n} \mid \mathbf{H}^{n}, \widetilde{\mathbf{X}}^{n}} \sim \mathcal{C N}\left(\operatorname{vec}\left(\frac{1}{\sqrt{K}} \mathbf{H}^{n} \widetilde{\mathbf{X}}^{n}\right), \sigma^{2} \mathbf{I}_{N \times n}\right)
\end{aligned}
$$

where the function $\operatorname{vec}(\mathbf{A})$ vectorizes the matrix $\mathbf{A}$. Using $D\left(\mathcal{C N}\left(\mathbf{m}_{1}, \sigma^{2} \mathbf{I}\right) \| \mathcal{C N}\left(\mathbf{m}_{2}, \sigma^{2} \mathbf{I}\right)\right)=\left\|\mathbf{m}_{1}-\mathbf{m}_{2}\right\|^{2} / \sigma^{2}$ together with (49) and (50) in (48), we obtain from standard computations

$$
\begin{aligned}
& \mathbb{E}_{H^{n}, \widetilde{X}^{n}}\left[D\left(\mathbb{P}_{Y^{n} \mid \mathbf{H}^{n}=H^{n}, \widetilde{\mathbf{X}}^{n}=\widetilde{X}^{n}} \| \mathbb{P}_{\widetilde{Y}^{n} \mid \mathbf{H}^{n}=H^{n}, \widetilde{\mathbf{X}}^{n}=\widetilde{X}^{n}}\right)\right] \\
& =\mathbb{E}_{H^{n}, \widetilde{X}^{n}}\left[\frac{1}{\sigma^{2} K}\left|\frac{\sqrt{n K}}{\left\|\widetilde{X}^{n}\right\|_{F}}-1\right|^{2}\left\|H^{n} \widetilde{X}^{n}\right\|_{F}^{2}\right] \\
& =\frac{1}{\sigma^{2}} \frac{N}{K} \mathbb{E}\left[\left|\sqrt{n K}-\left\|\widetilde{X}^{n}\right\|_{F}\right|^{2}\right] \\
& =\frac{1}{\sigma^{2}} \frac{N}{K}\left(2 n K-2 \sqrt{n K} \mathbb{E}\left[\left\|\widetilde{X}^{n}\right\|_{F}\right]\right) \text {. }
\end{aligned}
$$

Now, since $\tilde{X}^{n}$ is Gaussian, $\sqrt{2}\left\|\tilde{X}^{n}\right\|_{F}$ is $\chi_{2 n K}$-distributed, so that

$$
\mathbb{E}\left[\left\|\widetilde{X}^{n}\right\|_{F}\right]=\frac{\Gamma(n K+1 / 2)}{\Gamma(n K)} .
$$

Using this result in (53) leads to

$$
\begin{aligned}
\text { (53) } & =\frac{2 n N}{\sigma^{2}}\left(1-\frac{\Gamma(n K+1 / 2)}{\sqrt{n K} \Gamma(n K)}\right) \\
& \leq \frac{2 n N}{\sigma^{2}}\left(1-\sqrt{\frac{n K}{n K+1 / 2}}\right) \\
& =\frac{2 n N}{\sigma^{2}}\left(1-\sqrt{1-\frac{1 / 2}{n K+1 / 2}}\right) \\
& =\frac{2 n N}{\sigma^{2}}(1-1+\mathcal{O}(1 /(n K)))=\mathcal{O}(1)
\end{aligned}
$$

where we used in (56) that for $a \in(0,1)$ and $x>0$ [27]

$$
1 \geq \frac{\Gamma(x+a)}{\Gamma(x) x^{a}} \geq\left(\frac{x}{x+a}\right)^{1-a}
$$

and (58) follows because $\sqrt{1+x}=1+\mathcal{O}(x)$ as $x \rightarrow 0$.

With this result at hand, we can prove the following result.

Proposition 1 (Bounds on the average error probability):

The following two statements hold:

(i) Lower bound: Let $Y_{+}^{n} \in \mathbb{C}^{N \times(n+1)}$ denote the random variable associated to the output of the channel $\mathbb{P}_{Y_{+}^{n} \mid X_{+}^{n}, H^{n}}$ corresponding to the input $X_{+}^{n} \in \mathbb{C}^{K \times(n+1)}$ and fading $H^{n} \in \mathbb{C}^{K \times N}$. Then, 60 on the top of the next page holds, where $\mathbb{Q}_{n,+}\left(\cdot \mid H^{n}\right)$ is an $H^{n}$-measurable random variable taking values in $\mathcal{P}\left(\mathbb{C}^{N \times(n+1)}\right)$ and

$$
\mathcal{S}_{=}^{n,+}=\left\{\mathbf{X}_{+}^{n} \in \mathbb{C}^{K \times(n+1)} \mid \frac{1}{(n+1) K} \operatorname{tr} \mathbf{X}_{+}^{n}\left(\mathbf{X}_{+}^{n}\right)^{\mathrm{H}}=1\right\} .
$$




$$
\begin{aligned}
\mathbb{P}_{e}(r \mid \beta, c) & \geq \mathbb{F}(r \mid \beta, c) \\
& \triangleq \inf _{\substack{\left\{\mathbb{P}_{\left.X_{+}^{n}\right\}_{n=1}^{\infty}} \\
\mathbb{P}_{X_{+}^{n} \in \mathcal{P}\left(\mathcal{S}_{=}^{n+1}\right)}\right.}} \sup _{\left.\mathbb{Q}_{n,+}\right\}_{n=1}^{\infty}} \lim _{\xi \downarrow 0} \limsup _{n \stackrel{(\beta, c)}{\longrightarrow \infty}} \operatorname{Pr}\left[\sqrt{n K}\left(\frac{1}{n K} \log \frac{\left.\left.\mathbb{P}_{Y_{+}^{n} \mid X_{+}^{n}, H^{n}\left(d Y_{+}^{n} \mid X_{+}^{n}, H^{n}\right)}-C\right) \leq r-\xi\right]}{\mathbb{Q}_{n,+}\left(d Y_{+}^{n} \mid H^{n}\right)}-C\right]\right.
\end{aligned}
$$

$$
\mathbb{P}_{e}(r \mid \beta, c) \leq \mathbb{G}(r \mid \beta, c) \triangleq \lim _{\xi \downarrow 0} \limsup _{n \stackrel{(\beta, c)}{\longrightarrow} \infty} \operatorname{Pr}\left[\sqrt{n K}\left(\frac{1}{n K} \log \frac{\mathbb{P}_{Y^{n} \mid X^{n}, H^{n}}\left(d Y^{n} \mid X^{n}, H^{n}\right)}{\widetilde{\mathbb{P}}_{n}\left(d Y^{n} \mid H^{n}\right)}-C\right) \leq r+\xi\right]
$$

(ii) Upper bound: There exists a codebook of size $M_{n}$ with codewords of block-length $n$ that together with the ML decoder form a $\left(P_{e}^{(n)}, M_{n}\right)$-code $\mathcal{C}_{n}$ such that, for all real $r$, (62) on the top of the next page holds, which is computed from the probability measure induced by inputs uniformly distributed over the power shell:

$$
\mathbb{P}_{X^{n}}\left(\mathbf{X}^{n}\right)=\frac{\mathbb{1}\left[\operatorname{tr} \mathbf{X}^{n}\left(\mathbf{X}^{n}\right)^{\mathrm{H}}=n K\right]}{S_{2 n K}(\sqrt{n K})}
$$

which satisfy $\mathbb{P}_{X^{n}}\left(\mathcal{S}_{=}^{n}\right)=1$, and where $S_{2 n K}(r)=$ $2 \pi^{n K} \Gamma(n K)^{-1} r^{2 n K-1}$ is the surface area of a $2 n K$ dimensional sphere of radius $r$, and $\widetilde{\mathbb{P}}_{n}$ is the output distribution of the channel $\mathbb{P}_{Y^{n} \mid X^{n}, H^{n}}$ induced by a complex Gaussian input distribution with zero mean and covariance $\mathbf{I}_{K n}$.

Proof: This proof is segmented in two parts. We first derive error probability bounds for each $N, K, n$, based on the established slight variations on the Verdú-Han's Lemma 1 and the modified Feinstein's Lemma 2 and then bringing $N, K, n$ to infinity leads to Proposition 1.

We first start with the proof of the lower bound 60). Let $\mathcal{C}_{n}$ be a $\left(P_{e}^{(n)}, M_{n}\right)$-code whose probability measure satisfies $\mathbb{P}_{X^{n}} \in \mathcal{P}\left(\mathcal{S}^{n}\right)$. From this code, following the approach in [9], we define the code $\mathcal{C}_{n,+}$ with codewords $\left\{\mathbf{X}_{i,+}^{n}=\right.$ $\left.\left[\mathbf{X}_{i}^{n}, \mathbf{x}_{i}\right], i=1, \ldots, M_{n}\right\}$, where $\left\{\mathbf{X}_{i}^{n}, i=1, \ldots, M_{n}\right\}=$ $\operatorname{Supp}\left(\mathcal{C}_{n}\right)$ and $\mathbf{x}_{i}$ satisfies $\left\|\mathbf{x}_{i}\right\|^{2}=(n+1) K-\operatorname{tr} \mathbf{X}_{i}^{n}\left(\mathbf{X}_{i}^{n}\right)^{\mathrm{H}}$, and with the same decision region as for $\mathcal{C}_{n}$ discarding the last channel output (corresponding to input $\mathbf{x}_{i}$ ). Note that the probability measure $\mathbb{P}_{X_{+}^{n}}$ of the code $\mathcal{C}_{n,+}$ satisfies $\mathbb{P}_{X_{+}^{n}} \in \mathcal{P}\left(\mathcal{S}_{=}^{n,+}\right)$ and that $P_{e}^{(n)}\left(\mathcal{C}_{n,+}^{+}\right)=P_{e}^{(n)}\left(\mathcal{C}_{n}\right)$.

From Lemma 1 the average error probability must satisfy

$$
\begin{aligned}
& P_{e}^{(n)}\left(\mathcal{C}_{n}\right)=P_{e}^{(n)}\left(\mathcal{C}_{+, n}\right) \\
& \geq \operatorname{Pr}\left[\log \frac{\mathbb{P}_{+}^{n} \mid X_{+}^{n}, H^{n}}{\mathbb{Q}_{+, n}\left(d Y_{+}^{n} \mid X_{+}^{n}, H^{n}\right)} \leq \log \gamma\right]-\frac{\gamma}{M_{n}}
\end{aligned}
$$

for each $n=1,2, \ldots, \gamma>0$, where $\mathbb{Q}_{+, n}\left(\cdot \mid H^{n}\right)$ is $H^{n_{-}}$ measurable and takes values in $\mathcal{P}\left(\mathbb{C}^{N \times(n+1)}\right)$, with $Y_{+}^{n}=$ $\frac{1}{\sqrt{K}} H^{n} X_{+}^{n}+\sigma W_{+}^{n}, W_{+}^{n} \in \mathbb{C}^{N \times(n+1)}$ with i.i.d. $\mathcal{C N}(0,1)$ entries. Let us choose $\gamma$ as

$$
\frac{1}{n K} \log \gamma=\frac{1}{n K} \log M_{n}-\frac{\xi}{\sqrt{n K}}
$$

for some $\xi>0$. We now set the coding rate

$$
\frac{1}{n K} \log M_{n}=C+\frac{r}{\sqrt{n K}}
$$

for some real $r$. Then, combining (64)- w6 wain

$$
\begin{aligned}
& P_{e}^{(n)}\left(\mathcal{C}_{n}\right) \geq \\
& \operatorname{Pr}\left[\sqrt{n K}\left(\frac{1}{n K} \log \frac{\mathbb{P}_{Y_{+}^{n} \mid X_{+}^{n}, H^{n}}\left(d Y^{n} \mid X_{+}^{n}, H^{n}\right)}{\mathbb{Q}_{+, n}\left(d Y_{+}^{n} \mid H^{n}\right)}-C\right)\right. \\
& \quad \leq r-\xi]-\exp (-\sqrt{n K} \xi)
\end{aligned}
$$

Taking the limit superior over $n$ on the last equation, we obtain

$$
\begin{aligned}
& \limsup _{(\beta, c)} P_{e}^{(n)}\left(\mathcal{C}_{n}\right) \geq \underset{n \stackrel{(\beta, c)}{\longrightarrow} \infty}{\limsup \operatorname{Pr}}[\sqrt{n K} \\
& \left.\times\left(\frac{1}{n K} \log \frac{\mathbb{P}_{Y_{+}^{n} \mid X_{+}^{n}, H^{n}\left(d Y_{+}^{n} \mid X_{+}^{n}, H^{n}\right)}}{\mathbb{Q}_{+, n}\left(d Y_{+}^{n} \mid H^{n}\right)}-C\right) \leq r-\xi\right] .
\end{aligned}
$$

As this is true for each $\xi>0$ and $\mathbb{Q}_{+, n}$ as defined above, we can take $\xi \downarrow 0$ followed by the supremum over $\mathbb{Q}_{+, n}$ on the RHS of (68). Taking then the infimum over the codes on the RHS then LHS, we conclude that

$$
\mathbb{P}_{e}(r \mid \beta, c) \geq \mathbb{F}(r \mid \beta, c)
$$

which proves part $(i)$ of the proposition.

We now prove part $(i i)$ for the upper bound in 62). From Lemma 2, we know that there exists a $\left(P_{e}^{(n)}, M_{n}\right)$-code $\mathcal{C}_{n}$ whose average error probability satisfies

$$
\begin{aligned}
P_{e}^{(n)}\left(\mathcal{C}_{n}\right) \leq \inf _{\gamma>0}\left\{\operatorname { P r } \left[\frac{1}{n K} \log \frac{\mathbb{P}_{Y^{n} \mid X^{n}, H^{n}}\left(d Y^{n} \mid X^{n}, H^{n}\right)}{\widetilde{\mathbb{P}}_{n}\left(d Y^{n} \mid H^{n}\right)}\right.\right. \\
\left.\left.\leq \frac{1}{n K} \log \left(\gamma \kappa_{n}\right)\right]+\frac{M_{n}}{\gamma}\right\}+\alpha_{n}
\end{aligned}
$$

for every $n=1,2, \ldots$, where $\alpha_{n}$ is defined as in Lemma 3 . Let us now set

$$
\frac{1}{n K} \log \gamma=\frac{1}{n K} \log M_{n}+\frac{\xi}{\sqrt{n K}}
$$

for some $\xi>0$. Then, we have the following chain of 
inequalities:

$$
\begin{aligned}
& P_{e}^{(n)}\left(\mathcal{C}_{n}\right) \leq \operatorname{Pr}[ \frac{1}{n K} \log \frac{\mathbb{P}_{Y^{n} \mid X^{n}, H^{n}}\left(d Y^{n} \mid X^{n}, H^{n}\right)}{\widetilde{\mathbb{P}}_{n}\left(d Y^{n} \mid H^{n}\right)} \\
&\left.\leq \frac{1}{n K} \log \gamma+\frac{1}{n K} \log \kappa_{n}\right]+\frac{M_{n}}{\gamma}+\alpha_{n} \\
&=\operatorname{Pr}\left[\frac{1}{n K} \log \frac{\mathbb{P}_{Y^{n} \mid X^{n}, H^{n}}\left(d Y^{n} \mid X^{n}, H^{n}\right)}{\widetilde{\mathbb{P}}_{n}\left(d Y^{n} \mid H^{n}\right)}\right. \\
&\left.\leq \frac{1}{n K} \log M_{n}+\frac{\xi}{\sqrt{n K}}+\frac{1}{n K} \log \kappa_{n}\right] \\
&+\exp (-\sqrt{n K} \xi)+\alpha_{n}
\end{aligned}
$$

which simply follows by replacing (71) in (70). For some $r$ real, we choose the coding rate

$$
\frac{1}{n K} \log M_{n}=C+\frac{r}{\sqrt{n K}} .
$$

By combining (70) and (73), taking the superior limit on $n$, then $\xi \downarrow 0$ on the RHS, and the infimum over the codes on the LHS, we obtain

$$
\begin{aligned}
& \mathbb{P}_{e}(r \mid \beta, c) \leq \lim _{\xi \downarrow 0} \limsup _{n \underset{(\beta, c)}{\longrightarrow} \infty} \operatorname{Pr}[\sqrt{n K} \\
& \left.\times\left(\frac{1}{n K} \log \frac{\mathbb{P}_{Y^{n} \mid X^{n}, H^{n}}\left(d Y^{n} \mid X^{n}, H^{n}\right)}{\widetilde{\mathbb{P}}_{n}\left(d Y^{n} \mid H^{n}\right)}-C\right) \leq r+\xi\right]
\end{aligned}
$$

where we used $\alpha_{n} \rightarrow 0$ while $\kappa_{n} \rightarrow \infty$, such that $\frac{1}{\sqrt{n K}} \log \kappa_{n} \rightarrow 0$. This concludes the proof.

\section{APPENDIX B}

\section{PROOF OF THEOREM 3}

The proof relies on information spectrum methods [19] and is more exactly related to Hayashi's proof-techniques used in [8]. Our starting point is Proposition 1 in Appendix $\mathrm{A}$ which relates the optimal average error probability $\mathbb{P}_{e}(r \mid \beta, c)$ to the statistics of the mutual information density.

The main problem in studying the optimal average error probability lies in the difficulty to perform any analytical calculus on the information spectrum of $\mathbb{P}_{Y^{n} \mid X^{n}, H^{n}}$, unless the underlying distributions (of $X^{n}, Y^{n} \mid \mathbf{X}^{n}, H^{n}$, or $Y^{n} \mid H^{n}$ ) are Gaussian. Proposition 1 precisely handles this difficulty. Indeed, first note that the lower bound (60) can be further bounded by the same expression with $\mathbb{Q}_{n,+}$ chosen to be Gaussian with appropriate mean and variance. As for (62), it already features an information spectrum of Gaussian distributions. Both lower and upper bounds will thus rely on exploiting Theorem 2 with the major difference that, while the upper bound from (62) provides a definite choice for $\mathbb{P}_{X^{n}}$ that allows for an accurate control of the variance $\theta_{n}$ of Theorem 2, (60) does not and will force us to consider the worst case scenario where $\frac{1}{K} \operatorname{tr}\left(\mathbf{A}^{n}\right)^{2}=0$, with $\mathbf{A}^{n}=\mathbf{I}_{N}-\frac{1}{n} \mathbf{X}^{n}\left(\mathbf{X}^{n}\right)^{\mathrm{H}}$. As briefly discussed in Section III] the term $\left(\mathbf{A}^{n}\right)^{2}$ appears due to the randomness in the channel $H^{n}$, leaving the problem of non-matching upper and lower bounds; this is unlike the previously studied AWGN scenarios (e.g., [8], [9]) where $H^{n}=\mathbf{I}_{N}$ and only terms in $\mathbf{A}^{n}$ but not $\left(\mathbf{A}^{n}\right)^{2}$ account for the second-order statistics.

\section{A. Proof of the lower bound on the optimal average error probability}

From (60),

$$
\begin{aligned}
& \mathbb{P}_{e}(r \mid \beta, c) \geq \inf _{\substack{\left\{\mathbb{P}_{X_{+}^{n}}\right\}_{n=1}^{\infty} \\
\mathbb{P}_{X_{+}^{n}} \in \mathcal{P}\left(\mathcal{S}_{=}^{n+1}\right)}} \lim _{\xi \downarrow 0} \limsup _{n \stackrel{(\beta, c)}{\longrightarrow} \operatorname{Pr}}[\sqrt{n K} \\
& \left.\times\left(\frac{1}{n K} \log \frac{\mathbb{P}_{Y_{+}^{n} \mid X_{+}^{n}, H^{n}}\left(d Y_{+}^{n} \mid X_{+}^{n}, H^{n}\right)}{\mathbb{Q}_{n,+}\left(d Y_{+}^{n}\right)}-C\right) \leq r-\xi\right]
\end{aligned}
$$

where, for fixed $\mathbf{H}^{n}, \mathbb{Q}_{n,+}$ is taken to be complex Gaussian with zero mean and covariance matrix $\frac{1}{K} \mathbf{H}^{n}\left(\mathbf{H}^{n}\right)^{\mathrm{H}}+\sigma^{2} \mathbf{I}_{N}$. Thus,

$$
\begin{aligned}
& \mathbb{P}_{e}(r \mid \beta, c) \geq \inf _{\left\{\mathbb{P}_{X_{+}^{n}} \in \mathcal{P}\left(\mathcal{S}_{=}^{n,+}\right)\right\}_{n=1}^{\infty}} \lim _{\xi \downarrow 0} \\
& \quad \limsup _{n \stackrel{(\beta, c)}{\longrightarrow} \operatorname{Pr}} \operatorname{Pr}\left[\sqrt{n K}\left(I_{N, K}^{X_{+}^{n}}-C\right) \leq r-\xi\right]
\end{aligned}
$$

where $I_{N, K}^{X_{+}^{n}}$ is defined in $(78)$ on the next page and where $W_{+}^{n} \in \mathbb{C}^{N \times(n+1)}$ is composed of i.i.d. $\mathcal{C N}(0,1)$ elements.

To proceed, we now call Theorem 2 for the random variable $I_{N, K}^{X_{+}^{n}}$. Let $\left\{X_{+}^{n}\right\}_{n=1}^{\infty}$ be a sequence with $X_{+}^{n}$ random with support in $\mathcal{S}_{=}^{n,+}$ for each $n$. Denoting $A_{+}^{n}=\mathbf{I}_{K}-\frac{1}{n+1} X_{+}^{n}\left(X_{+}^{n}\right)^{\mathrm{H}}$, for any real $z$, as $n \stackrel{(\beta, c)}{\longrightarrow} \infty$, we then have

$$
\operatorname{Pr}\left[\frac{\sqrt{n K}}{\tilde{\theta}_{n,+}}\left(I_{N, K}^{X_{+}^{n}}-C\right) \leq z\right] \rightarrow \Phi(z)
$$

where $\quad \tilde{\theta}_{n,+}^{2} \triangleq \frac{1}{1+n^{-1}}\left(\theta_{-}^{2}+\zeta \frac{1}{K} \operatorname{tr}\left(A_{+}^{n}\right)^{2}-\frac{1}{K} \log (1-\right.$ $\left.\left.c^{-1} \delta_{0}\left(\sigma^{2}\right)\left(1+\delta_{0}\left(\sigma^{2}\right)\right)^{-2}\right)-\frac{1}{K} \zeta \beta^{-1}\right)$, in which the terms in $K^{-1}$ or $n^{-1}$ arise from accounting for the fact that $X_{+}^{n} \in \mathbb{C}^{K \times(n+1)}$ and $W_{+}^{n} \in \mathbb{C}^{N \times(n+1)}$. But since $-\frac{1}{K} \log \left(1-c^{-1} \delta_{0}\left(\sigma^{2}\right)\left(1+\delta_{0}\left(\sigma^{2}\right)\right)^{-2}\right)-\frac{1}{K} \zeta \beta^{-1} \rightarrow 0$ as $n \stackrel{(\beta, c)}{\longrightarrow} \infty$, we have more simply by Slutsky's lemma

$$
\operatorname{Pr}\left[\frac{\sqrt{n K}}{\theta_{n,+}}\left(I_{N, K}^{X_{+}^{n}}-C\right) \leq z\right] \rightarrow \Phi(z)
$$

with $\theta_{n,+}^{2} \triangleq \theta_{-}^{2}+\zeta \frac{1}{K} \operatorname{tr}\left(A_{+}^{n}\right)^{2}$.

We can now write

$$
\begin{aligned}
& \operatorname{Pr}\left[\sqrt{n K}\left(I_{N, K}^{X_{+}^{n}}-C\right) \leq r-\xi\right] \\
& =\operatorname{Pr}\left[\frac{\sqrt{n K}}{\theta_{n,+}}\left(I_{N, K}^{X_{+}^{n}}-C\right) \leq \frac{r-\xi}{\theta_{n,+}}\right] \\
& \stackrel{(a)}{\geq} \begin{cases}\operatorname{Pr}\left[\frac{\sqrt{n K}}{\theta_{n,+}}\left(I_{N, K}^{\bar{X}_{+}^{n}}-C\right) \leq \frac{r-\xi}{\theta_{-}}\right] & , r \leq 0 \\
\operatorname{Pr}\left[\frac{\sqrt{n K}}{\theta_{n,+}}\left(I_{N, K}^{\bar{X}_{+}^{n}}-C\right) \leq 0\right] & , r>0\end{cases} \\
& = \begin{cases}\Phi\left(\frac{r-\xi}{\theta_{-}}\right)+\ell_{n} & , r \leq 0 \\
\frac{1}{2}+\ell_{n} & , r>0\end{cases}
\end{aligned}
$$




$$
\begin{aligned}
I_{N, K}^{X_{+}^{n}} \triangleq & \frac{1}{K} \log \operatorname{det}\left(\mathbf{I}_{N}+\frac{1}{\sigma^{2}} \frac{H^{n}\left(H^{n}\right)^{\mathrm{H}}}{K}\right) \\
& +\frac{1}{n K} \operatorname{tr}\left[\left(\frac{H^{n}\left(H^{n}\right)^{\mathrm{H}}}{K}+\sigma^{2} \mathbf{I}_{N}\right)^{-1}\left(\frac{H^{n}}{\sqrt{K}} X_{+}^{n}+\sigma W_{+}^{n}\right)\left(\frac{H^{n}}{\sqrt{K}} X_{+}^{n}+\sigma W_{+}^{n}\right)^{\mathrm{H}}-W_{+}^{n}\left(W_{+}^{n}\right)^{\mathrm{H}}\right]
\end{aligned}
$$

for some sequence $\ell_{n} \downarrow 0$, where $(a)$ holds since $\theta_{n,+} \geq \theta_{-}>$ 0 and since we took $r-\xi>0$ for $r>0$. The term $1 / 2$ arises from $\Phi(0)=1 / 2$ which originates from $\theta_{n}$ not being bounded from above since $\frac{1}{K} \operatorname{tr}\left(A_{+}^{n}\right)^{2}$ can grow like $\mathcal{O}(n)$.

Taking the limit superior as $n \stackrel{(\beta, c)}{\longrightarrow} \infty$ of the above equation leads to

$$
\begin{aligned}
& \limsup _{(\beta, c)} \operatorname{Pr}\left[\sqrt{n K}\left(I_{N, K}^{X_{+}^{n}}-C\right) \leq r-\xi\right] \\
& n \stackrel{(\beta, c)}{\longrightarrow} \infty \\
& \geq \begin{cases}\Phi\left(\frac{r-\xi}{\theta_{-}}\right) & , r \leq 0 \\
\frac{1}{2} & , r>0 .\end{cases}
\end{aligned}
$$

By continuity of $\Phi$, we can freely take the limit $\xi \downarrow 0$ on the right- then left-hand sides to obtain

$$
\begin{aligned}
& \lim _{\xi \downarrow 0} \limsup _{n \stackrel{(\beta, c)}{\longrightarrow} \infty} \operatorname{Pr}\left[\sqrt{n K}\left(I_{N, K}^{X_{+}^{n}}-C\right) \leq r-\xi\right] \\
& \geq \begin{cases}\Phi\left(\frac{r}{\theta_{-}}\right) & , r<0 \\
\frac{1}{2} & , r \geq 0 .\end{cases}
\end{aligned}
$$

Equation 85 is valid regardless of the choice of the sequence $\left\{\mathbb{P}_{X_{+}^{n}} \in \mathcal{P}\left(\mathcal{S}_{=}^{n,+}\right)\right\}_{n=1}^{\infty}$. This therefore implies

$$
\mathbb{P}_{e}(r \mid \beta, c) \geq \begin{cases}\Phi\left(\frac{r}{\theta_{-}}\right) & , r<0 \\ \frac{1}{2} & , r \geq 0\end{cases}
$$

which completes the proof.

\section{B. Proof of the upper bound on the optimal average error probability}

From (62), we recall that

$$
\begin{aligned}
& \mathbb{P}_{e}(r \mid \beta, c) \leq \lim _{\xi \downarrow 0} \limsup _{n \stackrel{(\beta, c)}{\longrightarrow} \infty} \operatorname{Pr}[\sqrt{n K} \times \\
& \left.\left(\frac{1}{n K} \log \frac{\mathbb{P}_{Y^{n} \mid X^{n}, H^{n}}\left(d Y^{n} \mid X^{n}, H^{n}\right)}{\widetilde{\mathbb{P}}_{n}\left(d Y^{n} \mid H^{n}\right)}-C\right) \leq r+\xi\right]
\end{aligned}
$$

where $\widetilde{\mathbb{P}}_{n}\left(\cdot \mid H^{n}\right)$ is a Gaussian random variable with zero mean and covariance $H^{n}\left(H^{n}\right)^{\mathrm{H}}+\sigma^{2} \mathbf{I}_{N}$ and the outer probability is taken over $H^{n}$ and over the random variable $X^{n}$ having uniform distribution $\mathbb{P}_{X^{n}}$ over the sphere $\mathcal{S}_{=}^{n}$, as per 63. Denoting, similar to above,

$$
I_{N, K}^{X^{n}}=\frac{1}{n K} \log \frac{\mathbb{P}_{Y^{n} \mid X^{n}, H^{n}}\left(d Y^{n} \mid X^{n}, H^{n}\right)}{\widetilde{\mathbb{P}}_{n}\left(d Y^{n} \mid H^{n}\right)}
$$

we get from the Gaussianity of both $\mathbb{P}_{Y^{n} \mid X^{n}, H^{n}}$ and $\widetilde{\mathbb{P}}_{n}$ that $I_{N, K}^{X^{n}}$ is given by (19) with $X^{n}$ of law $\mathbb{P}_{X^{n}}$, while $H^{n}$ and $W^{n}$ are zero mean Gaussian with (properly normalized) unit covariance.
Once again, we resort to Theorem 2 to determine the limiting behavior of $I_{N, K}^{X^{n}}$. As opposed to the lower bound, where $\mathbb{P}_{X^{n}} \in \mathcal{P}\left(\mathcal{S}_{=}^{n}\right)$ was left undefined, $\mathbb{P}_{X^{n}}$ is now fixed and will allow for a more accurate control of the limiting variance of $I_{N, K}^{X^{n}}$. We first obtain

$$
\begin{aligned}
& \limsup _{(\beta, c)}^{\longrightarrow} \operatorname{Pr}\left[\sqrt{n K}\left(I_{N, K}^{X^{n}}-C\right) \leq r+\xi\right] \\
& =\limsup _{n \stackrel{(\beta, c)}{\longrightarrow} \infty} \operatorname{Pr}\left[\frac{\sqrt{n K}}{\theta_{n}}\left(I_{N, K}^{X^{n}}-C\right) \leq \frac{r+\xi}{\theta_{n}}\right]
\end{aligned}
$$

where $\theta_{n}$ is defined in 20, where we recall that $A^{n}=\mathbf{I}_{N}$ $\frac{1}{n} X^{n}\left(X^{n}\right)^{\mathrm{H}}$. Now, it appears that

$$
\frac{1}{K} \operatorname{tr}\left[\left(A^{n}\right)^{2}\right] \stackrel{(\beta, c)}{\longrightarrow} \frac{1}{\beta}
$$

almost surely. To obtain this result, it suffices to realize that $X^{n}=\bar{X}^{n}\left(\frac{1}{N K} \operatorname{tr} \bar{X}^{n}\left(\bar{X}^{n}\right)^{\mathrm{H}}\right)^{-\frac{1}{2}}$ for $\bar{X}^{n} \in \mathbb{C}^{K \times n}$ a standard Gaussian random matrix with entries of zero mean and unit variance; from classical random matrix results (that may be obtained by means of the Gaussian tools defined in Appendix [C), we have that $\frac{1}{N K} \operatorname{tr} \bar{X}^{n}\left(\bar{X}^{n}\right)^{\mathrm{H}} \rightarrow 1$ while $\frac{1}{N K} \operatorname{tr}\left(\bar{X}^{n}\left(\bar{X}^{n}\right)^{\mathrm{H}}\right)^{2} \rightarrow 1+\beta^{-1}$, almost surely; plugging these results in the expression of $\frac{1}{K} \operatorname{tr}\left(A^{n}\right)^{2}$ gives the expected result. As such, we now have that $\theta_{n} \stackrel{(\beta, c)}{\longrightarrow} \theta_{+}$almost surely (and so in probability), with $\theta_{+}$defined in Theorem 3 . By Slutsky's lemma and Theorem 2, we thus have

$$
\begin{aligned}
& \limsup _{n \stackrel{(\beta, c)}{\longrightarrow} \infty} \operatorname{Pr}\left[\sqrt{n K}\left(I_{N, K}^{X^{n}}-C\right) \leq r+\xi\right] \\
& =\limsup _{(\beta, c)} \operatorname{Pr}\left[\frac{\sqrt{n K}}{\theta_{n}}\left(I_{N, K}^{X^{n}}-C\right) \leq \frac{r+\xi}{\theta_{+}}\right] \\
& =\Phi\left(\frac{r+\xi}{\theta_{+}}\right)
\end{aligned}
$$

which, along with the fact that

$$
\lim _{\xi \downarrow 0} \Phi\left(\frac{r+\xi}{\theta_{+}}\right)=\Phi\left(\frac{r}{\theta_{+}}\right)
$$

concludes the proof.

\section{APPENDIX C \\ GAUSSIAN TOOLS AND RELATED RESULTS}

The CLT, Theorem 2, relies on advanced tools from random matrix theory along with standard linear algebraic relations which are constantly called for. This section introduces the random matrix concepts and collects the aforementioned relations. 
Lemma 4 (Some matrix inequalities): For two $N \times N$ matrices $\mathbf{A}$ and $\mathbf{B}$, the following holds

$$
\text { (i) }|\operatorname{tr} \mathbf{A B}| \leq \sqrt{\operatorname{tr} \mathbf{A A}^{\mathrm{H}} \operatorname{tr} \mathbf{B B}^{\mathrm{H}}} .
$$

If $\mathbf{A}$ is Hermitian nonnegative definite, it further holds that

$$
\begin{aligned}
& \text { (ii) } \quad|\operatorname{tr} \mathbf{A B}| \leq\|\mathbf{B}\| \operatorname{tr} \mathbf{A} \\
& \text { (iii) } \frac{1}{N} \operatorname{tr} \mathbf{A} \leq\|\mathbf{A}\| .
\end{aligned}
$$

Lemma 5 (Cauchy-Schwarz inequality): For two complex random variables $x$ and $y$,

$$
|\mathbb{E}[x y]| \leq \sqrt{\mathbb{E}\left[|x|^{2}\right]} \sqrt{\mathbb{E}\left[|y|^{2}\right]} .
$$

Remark 7 (Application of the Cauchy-Schwarz inequality): Consider two random variables $x$ and $y$. By the CauchySchwarz inequality,

$$
|\mathbb{E}[(x-\mathbb{E}[x])(y-\mathbb{E}[y])]| \leq \sqrt{\operatorname{Var}[x]} \sqrt{\operatorname{Var}[y]} .
$$

Thus,

$$
\begin{aligned}
|\mathbb{E}[x y]| & =|\mathbb{E}[x] \mathbb{E}[y]+\mathbb{E}[(x-\mathbb{E}[x])(y-\mathbb{E}[y])]| \\
& \leq|\mathbb{E}[x] \mathbb{E}[y]|+\sqrt{\operatorname{Var}[x]} \sqrt{\operatorname{Var}[y]} .
\end{aligned}
$$

Moreover, it follows that

$$
\begin{aligned}
& \operatorname{Var}[x+y] \\
& =\operatorname{Var}[x]+\operatorname{Var}[y]+2 \operatorname{Re}\{\mathbb{E}[(x-\mathbb{E}[x])(y-\mathbb{E}[y])]\} \\
& \leq \operatorname{Var}[x]+\operatorname{Var}[y]+2 \sqrt{\operatorname{Var}[x]} \sqrt{\operatorname{Var}[y]} \\
& =(\sqrt{\operatorname{Var}[x]}+\sqrt{\operatorname{Var}[y]})^{2} .
\end{aligned}
$$

Lemma 6 (Integration by parts formula [17, Equation (2.1.42)]): Let $x=\left[x_{1}, \ldots, x_{N}\right]^{\top} \sim \mathcal{C N}(\mathbf{0}, \mathbf{R})$ and let $f(x)=f\left(x_{1}, \ldots x_{N}, x_{1}^{*}, \ldots x_{N}^{*}\right)$ be a $\mathcal{C}^{1}$ complex function, polynomially bounded together with its derivatives. Then,

$$
\mathbb{E}\left[x_{i} f(x)\right]=\sum_{j=1}^{N} \mathbf{R}_{i j} \mathbb{E}\left[\frac{\partial f(x)}{\partial x_{j}^{*}}\right] .
$$
Remark 8 (Integration by parts formula for functionals of matrices with $\dot{\gamma} \cdot \vec{i})$ d. entries) $\frac{\partial \mathbf{Q}}{\partial \mathbf{H}_{i j}^{*}} \mathbf{C}=-\frac{1}{K}[\mathbf{Q C Q H}]_{i j}$
Let $f(W)$ be a $\mathcal{C}^{1}$ complex function of the elements of $W$ and $W^{*}$, polynomially bounded together with its derivatives, where $W$ has i.i.d. entries $W_{i j} \sim \mathcal{C} \mathcal{N}(0,1)$. Then

$$
\mathbb{E}\left[W_{i j} f(W)\right]=\mathbb{E}\left[\frac{\partial f(W)}{\partial W_{i j}^{*}}\right] .
$$

Lemma 7 (Poincaré-Nash Inequality [17 Propostion 2.1.6]): Let $x$ and $f(x)$ be defined as in Lemma 6 and let $\nabla_{x} f(x)=\left[\partial f(x) / \partial x_{1}, \ldots, \partial f(x) / \partial x_{N}\right]^{\top}$ and $\nabla_{x^{*}} f(x)=\left[\partial f(x) / \partial x_{1}^{*}, \ldots, \partial f(x) / \partial x_{N}^{*}\right]^{\top}$. Then,

$$
\begin{aligned}
\operatorname{Var}[f(x)] \leq \mathbb{E}[ & {\left[\nabla_{x} f(x)^{\top} \mathbf{R} \nabla_{x} f(x)^{*}\right] } \\
& +\mathbb{E}\left[\nabla_{x^{*}} f(x)^{\mathrm{H}} \mathbf{R} \nabla_{x^{*}} f(x)\right] .
\end{aligned}
$$

Remark 9 (Poincaré-Nash Inequality for functionals of matrices with i.i.d $\left[\begin{array}{c}1 \\ K\end{array}\right.$ trie $\left.\tilde{Q}^{n}(u)\right]=\tilde{\delta}_{0}(u)+\mathcal{O}\left(\frac{1}{u^{4} n^{2}}\right)$

Moreover, denote $\mathbf{Q}=\left(\frac{1}{K} \mathbf{H H}^{\mathrm{H}}+x \mathbf{I}_{N}\right)^{-1}$ and $\tilde{\mathbf{Q}}=$ $\left(\frac{1}{K} \mathbf{H}^{\mathrm{H}} \mathbf{H}+x \mathbf{I}_{K}\right)^{-1}$ for some $x>0$. Then,

$$
\begin{aligned}
\frac{\partial \mathbf{Q}_{p q}}{\partial \mathbf{H}_{i j}^{*}} & =-\frac{1}{K}[\mathbf{Q} \mathbf{H}]_{p j} \mathbf{Q}_{i q} \\
\frac{\partial \mathbf{Q}_{p q}}{\partial \mathbf{H}_{i j}} & =-\frac{1}{K}\left[\mathbf{H}^{\mathrm{H}} \mathbf{Q}\right]_{j q} \mathbf{Q}_{p i} \\
\frac{\partial \tilde{\mathbf{Q}}_{p q}}{\partial \mathbf{H}_{i j}^{*}} & =-\frac{1}{K} \tilde{\mathbf{Q}}_{p j}[\mathbf{H} \tilde{\mathbf{Q}}]_{i q} \\
\frac{\partial \tilde{\mathbf{Q}}_{p q}}{\partial \mathbf{H}_{i j}} & =-\frac{1}{K} \tilde{\mathbf{Q}}_{j q}\left[\tilde{\mathbf{Q}} \mathbf{H}^{\mathrm{H}}\right]_{p i} .
\end{aligned}
$$

Corollary 1: Let $\mathbf{H} \in \mathbb{C}^{N \times K}$ and $\mathbf{C} \in \mathbb{C}^{N \times N}$. Denote $\mathbf{Q}=\left(\frac{1}{K} \mathbf{H} \mathbf{H}^{\mathrm{H}}+x \mathbf{I}_{N}\right)^{-1}$ for some $x>0$. Then,

Proof: The proof follows directly from Lemma 8 and some straightforward calculus.

Theorem 4: Let $\left\{H^{n}\right\}_{n=1}^{\infty}$, where $H^{n} \in \mathbb{C}^{N \times K}$ has i.i.d. entries $H_{i j}^{n} \sim \mathcal{C} \mathcal{N}(0,1)$. For $u>0$, let $Q^{n}(u)=\left(\frac{1}{K} H^{n}\left(H^{n}\right)^{\mathrm{H}}+u \mathbf{I}_{N}\right)^{-1}$ and $\tilde{Q}^{n}(u)=$ $\left(\frac{1}{K}\left(H^{n}\right)^{\mathrm{H}} H^{n}+u \mathbf{I}_{K}\right)^{-1}$. Then, as $n \stackrel{(\beta, c)}{\longrightarrow} \infty$,

$$
\mathbb{E}\left[\frac{1}{K} \operatorname{tr} Q^{n}(u)\right]=\delta_{0}(u)+\mathcal{O}\left(\frac{1}{u^{4} n^{2}}\right)
$$


where

$$
\begin{aligned}
& \delta_{0}(u)=\frac{c-1}{2 u}-\frac{1}{2}+\frac{\sqrt{(1-c+u)^{2}+4 c u}}{2 u} \\
& \tilde{\delta}_{0}(u)=\delta_{0}(u)-\frac{c-1}{u} .
\end{aligned}
$$

Proof: The proof follows from a direct adaption of [17, Theorem 7.2.2] (see also [21, Theorem 3 and Proposition 5] for a more complex matrix model) along with a careful control of the dependence on $u$ in the bounds.

Remark 10: The function $s(z)=\frac{\delta_{0}(-z)}{c}$ for $z \in \mathbb{C} \backslash \mathbb{R}_{+}$ corresponds to the Stieltjes transform of the Marčenko-Pastur law, see e.g., [28, Chapter 3.2].

Property 1 (Some properties of $\delta_{0}(u)$ ): The function $\delta_{0}(u), u>0$, as defined in Theorem 4 satisfies

(iv) $\frac{\delta_{0}(u)}{1+\delta_{0}(u)}=c-u \delta_{0}(u)$

$$
\begin{aligned}
\delta_{0}(u) & >\frac{c}{(1+\sqrt{c})^{2}+u}>0 \\
\delta_{0}(u) & <\frac{c}{u} \\
\delta_{0}(u) & =\frac{c}{1-c+u\left(1+\delta_{0}(u)\right)}
\end{aligned}
$$

(v) $\frac{1}{1+\delta_{0}(u)}=1-c+u \delta_{0}(u)$

$$
\delta_{0}^{\prime}(x)=-\frac{\delta_{0}(x)\left(1+\delta_{0}(x)\right)}{1-c+x\left(1+2 \delta_{0}(x)\right)} .
$$

Proof: Properties $(i)-(i i i)$ are due to $\delta_{0}(u)=c m(-u)$, where $m(z)$ is the Stieltjes transform of the Marčenko-Pastur law with support in $\left[(1-\sqrt{c})^{2},(1+\sqrt{c})^{2}\right] \cup\{0\}$ (see Remark 10 in Appendix C). Property (iv) follows from (iii) since

$$
\begin{aligned}
\delta_{0}(u) & =\frac{c}{1-c+u\left(1+\delta_{0}(u)\right)} \\
\Longleftrightarrow \quad \delta_{0}(u) & =\left(1+\delta_{0}(u)\right) c-u \delta_{0}(u)\left(1+\delta_{0}(u)\right) \\
\Longleftrightarrow \frac{\delta_{0}(u)}{1+\delta_{0}(u)} & =c-u \delta_{0}(u)
\end{aligned}
$$

Property $(v)$ follows from $(i i i)$ and $(i v)$. Property $(v i)$ is obtained from the differentiation of

$$
c=\delta_{0}(x)(1-c+x)+x \delta_{0}(x)^{2}
$$

which follows from Property $($ iii).

Lemma 9: Let $\sigma^{2}, c>0$ and $\delta_{m}(x), m \geq 0$, be as defined in Proposition 4 in Appendix E-A Then,

$$
\begin{aligned}
& \text { (i) } \begin{aligned}
\int_{\sigma^{2}}^{\infty} & c \frac{1-c+2 u \delta_{0}(u)-\frac{u^{2}}{c} \delta_{0}(u)^{2}}{u\left(1-c+u\left(1+2 \delta_{0}(u)\right)\right)} d u=\log \left(1+\delta_{0}\left(\sigma^{2}\right)\right) \\
& -\frac{\delta_{0}\left(\sigma^{2}\right)}{1+\delta_{0}\left(\sigma^{2}\right)}+c \log \left(1+\frac{1}{\sigma^{2}} \frac{1}{1+\delta_{0}\left(\sigma^{2}\right)}\right) \\
\text { (ii) } \int_{\sigma^{2}}^{\infty} & \frac{\delta_{0}(u)-\sigma^{2} \delta_{1}(u)}{1-c+u\left(1+2 \delta_{0}(u)\right)} d u \\
= & -\log \left(1-\frac{1}{c} \frac{\delta_{0}\left(\sigma^{2}\right)^{2}}{\left(1+\delta_{0}\left(\sigma^{2}\right)\right)^{2}}\right)
\end{aligned}
\end{aligned}
$$

Proof: For the proof of part $(i)$, simply note that

$$
\begin{aligned}
& c \frac{1-c+2 u \delta_{0}(u)-\frac{u^{2}}{c} \delta_{0}(u)^{2}}{u\left(1-c+u\left(1+2 \delta_{0}(u)\right)\right)} \\
& =\frac{c}{u}-\frac{u \delta_{0}(u)^{2}+c}{1-c+u\left(1+2 \delta_{0}(u)\right)} \\
& =\frac{c}{u}-\frac{u \delta_{0}(u)^{2}+c}{u \delta_{0}(u)+\frac{c}{\delta_{0}(u)}} \\
& =\frac{c}{u}-\delta_{0}(u)
\end{aligned}
$$

where we used Property 1 (iii) in the second equality. The result then unfolds from Theorem 1

For part $(i i)$, we start with the following calculus:

$$
\begin{aligned}
& \int_{\sigma^{2}}^{\infty} \frac{\delta_{0}(u)-\sigma^{2} \delta_{1}(u)}{1-c+u\left(1+2 \delta_{0}(u)\right)} d u \\
& =\int_{\sigma^{2}}^{\infty}\left[\frac{\delta_{0}(u)}{1-c+u\left(1+2 \delta_{0}(u)\right)}+\right. \\
& \left.\frac{\sigma^{2} \delta_{0}(u)\left(1+\delta_{0}\left(\sigma^{2}\right)\right)}{\left(1-c+\sigma^{2}\left(1+\sigma^{2}\right)+u \delta_{0}(u)\right)\left(1-c+u\left(1+2 \delta_{0}(u)\right)\right)}\right] d u \\
& =\int_{\sigma^{2}}^{\infty}\left[-\frac{\delta_{0}(u) \delta_{0}^{\prime}(u)}{\delta_{0}(u)\left(1+\delta_{0}(u)\right)}\right. \\
& \left.+\frac{\sigma^{2} \delta_{0}^{\prime}(u)\left(1+\delta_{0}\left(\sigma^{2}\right)\right)}{1+\sigma^{2}\left(1+\delta_{0}\left(\sigma^{2}\right)\right)+\delta_{0}(u) \sigma^{2}\left(1+\delta_{0}\left(\sigma^{2}\right)\right)}\right] d u
\end{aligned}
$$

where in the first equality we developed the expression of $\delta_{1}(u)$ and in the second equality we introduced $\delta_{0}^{\prime}(u)$ in both numerators and used the relation by iterating the relation $x \delta_{0}(x)^{2}=c-\delta_{0}(x)(1-c+x)$ (from Property 1 (iii)) in the second denominator in order to maintain a degree one polynomial in $\delta_{0}(u)$. Writing $\delta_{0}(u) \delta_{0}^{\prime}(u)=\left[2 \delta_{0}(u) \delta_{0}^{\prime}(u)+\right.$ $\left.\delta_{0}^{\prime}(u)\right]-\delta_{0}^{\prime}(u)\left(1+\delta_{0}(u)\right)$ in the numerator of the first term, we then find

$$
\begin{aligned}
& \int_{\sigma^{2}}^{\infty} \frac{\delta_{0}(u)-\sigma^{2} \delta_{1}(u)}{1-c+u\left(1+2 \delta_{0}(u)\right)} d u \\
& =\int_{\sigma^{2}}^{\infty}\left[-\frac{2 \delta_{0}(u) \delta_{0}^{\prime}(u)+\delta_{0}^{\prime}(u)}{\delta_{0}(u)\left(1+\delta_{0}(u)\right)}+\frac{\delta_{0}^{\prime}(u)}{\delta_{0}(u)}\right. \\
& \left.\quad+\frac{\sigma^{2} \delta_{0}^{\prime}(u)\left(1+\delta_{0}\left(\sigma^{2}\right)\right)}{1+\sigma^{2}\left(1+\delta_{0}\left(\sigma^{2}\right)\right)+\delta_{0}(u) \sigma^{2}\left(1+\delta_{0}\left(\sigma^{2}\right)\right)}\right] d u \\
& =\left[-\log \left(1+\delta_{0}(u)\right)\right. \\
& \left.\quad+\log \left(1+\sigma^{2}\left(1+\delta_{0}\left(\sigma^{2}\right)\right)\left(1+\delta_{0}(u)\right)\right)\right]_{u=\sigma^{2}}^{\infty} \\
& =\log \left(1+\delta_{0}\left(\sigma^{2}\right)\right)+\log \left(1+\sigma^{2}\left(1+\delta_{0}\left(\sigma^{2}\right)\right)\right) \\
& \quad-\log \left(1+\sigma^{2}\left(1+\delta_{0}\left(\sigma^{2}\right)\right)^{2}\right) \\
& =\log \left(\frac{\left(1+\delta_{0}\left(\sigma^{2}\right)\right)\left(1+\sigma^{2}\left(1+\delta_{0}\left(\sigma^{2}\right)\right)\right)}{1+\sigma^{2}\left(1+\delta_{0}\left(\sigma^{2}\right)\right)^{2}}\right) .
\end{aligned}
$$


At this point, remark that

$$
\begin{aligned}
& \frac{\left(1+\delta_{0}\left(\sigma^{2}\right)\right)\left(1+\sigma^{2}\left(1+\delta_{0}\left(\sigma^{2}\right)\right)\right)}{1+\sigma^{2}\left(1+\delta_{0}\left(\sigma^{2}\right)\right)^{2}} \\
& =1-\frac{\delta_{0}\left(\sigma^{2}\right)}{1+\sigma^{2}\left(1+\delta_{0}\left(\sigma^{2}\right)\right)^{2}}
\end{aligned}
$$

and that

$$
\begin{aligned}
1+\sigma^{2}\left(1+\delta_{0}\left(\sigma^{2}\right)\right)^{2} & =1+\sigma^{2}+\sigma^{2} \delta_{0}\left(\sigma^{2}\right)+c+c \delta_{0}\left(\sigma^{2}\right) \\
& =\frac{c}{\delta_{0}\left(\sigma^{2}\right)}+2 c+c \delta_{0}\left(\sigma^{2}\right) \\
& =c \frac{\left(1+\delta_{0}\left(\sigma^{2}\right)\right)^{2}}{\delta_{0}\left(\sigma^{2}\right)}
\end{aligned}
$$

using Property 1 (iii) in the second equality.

This allows us to finally conclude that

$$
\begin{aligned}
& \int_{\sigma^{2}}^{\infty} \frac{\delta_{0}(u)-\sigma^{2} \delta_{1}(u)}{1-c+u\left(1+2 \delta_{0}(u)\right)} d u \\
& =-\log \left(1-\frac{1}{c} \frac{\delta_{0}\left(\sigma^{2}\right)^{2}}{\left(1+\delta_{0}\left(\sigma^{2}\right)\right)^{2}}\right) .
\end{aligned}
$$

\section{APPENDIX D}

\section{PROOFS OF THE MAIN RANDOM MATRIX RESULTS}

In the proof of Theorem 2, we fundamentally rely on the fact that the random matrices $W^{n}$ and $H^{n}$ are Gaussian by assumption. This allows us to use the powerful integration-byparts and Poincaré-Nash inequalities (Lemma 6 and Lemma 7 in Appendix C) to compute the expectation and bound the variance of functionals of Gaussian variables. The derivation of Theorem 2 is specifically based on the characteristic function approach as explained in great detail in [21], [17].

This appendix is structured as follows: In Appendix D-A we introduce some additional notations and useful identities. We then prove Theorem 2 in Appendix D-B

\section{A. Preliminaries}

For readability, we often drop the index $n$ in matrix notations when there is no confusion, e.g., we write $H$ instead of $H^{n}$.

We start with the definition of two matrices, the so-called "resolvents" of $K^{-1} H H^{\mathrm{H}}$ and $K^{-1} H^{\mathrm{H}} H$, respectively, which will be of repeated use:

$$
\begin{aligned}
& Q(x)=\left(\frac{1}{K} H H^{\mathrm{H}}+x \mathbf{I}_{N}\right)^{-1} \in \mathbb{C}^{N \times N} \\
& \tilde{Q}(x)=\left(\frac{1}{K} H^{\mathrm{H}} H+x \mathbf{I}_{K}\right)^{-1} \in \mathbb{C}^{K \times K}
\end{aligned}
$$

for $x>0$. One can easily verify that:

$$
Q(x) \frac{H H^{\mathrm{H}}}{K}=\mathbf{I}_{N}-x Q(x), \quad \tilde{Q}(x) \frac{H^{\mathrm{H}} H}{K}=\mathbf{I}_{K}-x \tilde{Q}(x) .
$$

We will also rely several times on the following identities:

$$
\begin{aligned}
Q(x) H & =H \tilde{Q}(x), & & \tilde{Q}(x) H^{\mathrm{H}}=H^{\mathrm{H}} Q(x) \\
Q(x) \frac{H H^{\mathrm{H}}}{K} & =\frac{H H^{\mathrm{H}}}{K} Q(x), & & \tilde{Q}(x) \frac{H^{\mathrm{H}} H}{K}=\frac{H^{\mathrm{H}} H}{K} \tilde{Q}(x) \\
Q(x) Q(y) & =Q(y) Q(x), & & \tilde{Q}(x) \tilde{Q}(y)=\tilde{Q}(y) \tilde{Q}(x) .
\end{aligned}
$$

Using the above relations, it is easy to prove the following bounds on the spectral norm:

$$
\begin{aligned}
\|Q(x)\|=\|\tilde{Q}(x)\| & \leq \frac{1}{x} \\
\left\|Q(x) \frac{H H^{\mathrm{H}}}{K}\right\|=\left\|\tilde{Q}(x) \frac{H^{\mathrm{H}} H}{K}\right\| & \leq 1 .
\end{aligned}
$$

\section{B. Proof of Theorem 2}

Outline of the proof:

The central object of Theorem 2 is the real quantity

$$
\begin{aligned}
& \Gamma_{n} \triangleq \sqrt{n K} I_{N, K}^{X_{+}^{n}} \\
& =\sqrt{\frac{n}{K}} \log \operatorname{det}\left(\mathbf{I}_{N}+\frac{1}{\sigma^{2}} \frac{1}{K} H H^{\mathrm{H}}\right) \\
& +\frac{1}{\sqrt{n K}} \operatorname{tr} Q\left(\sigma^{2}\right)\left(\frac{1}{\sqrt{K}} H X+\sigma W\right)\left(\frac{1}{\sqrt{K}} H X+\sigma W\right)^{\mathrm{H}} \\
& -\frac{1}{\sqrt{n K}} \operatorname{tr} W W^{\mathrm{H}}
\end{aligned}
$$

where $I_{N, K}^{X_{+}^{n}}$ was defined in (78). We also recall the dimensions $H \in \mathbb{C}^{N \times K}, X \in \mathbb{C}^{K \times n}$, and $W \in \mathbb{C}^{N \times n}$. Moreover, $X \in$ $\mathcal{S}_{=}^{n}$, where $\mathcal{S}_{=}^{n}$ was defined in (4).

It is our goal to prove that, under the hypotheses of the theorem,

$$
\tilde{\phi}_{n}(t) \triangleq \mathbb{E}\left[e^{\frac{\mathrm{i} t}{\theta_{n}}\left(\Gamma_{n}-\mu_{n}\right)}\right] \rightarrow e^{-\frac{t^{2}}{2}}
$$

for $t \in \mathbb{R}$ as $n \stackrel{(\beta, c)}{\longrightarrow} \infty$, where $\mu_{n} \triangleq \sqrt{n K} C$. This will imply, by Lévy's continuity theorem [18, Theorem 16.3], that

$$
\theta_{n}^{-1}\left(\Gamma_{n}-\mu_{n}\right) \Rightarrow \mathcal{N}(0,1)
$$

which is equivalent to the statement of the theorem. The main difficulty arises from the evaluation of the expectation in (160) which must be taken with respect to the three random matrices $W, H$, and $X$. Since the direct computation of $\tilde{\phi}_{n}(t)$ is intractable, we calculate its derivative with respect to $t$, leading to a differential equation which must be integrated. In order to further simplify the analysis, we split the computation of the expectation in three steps by successively considering the conditional expectations with respect to each of the matrices. These expectations are developed by the integration by parts formula (Lemma 6 in Appendix C) which yields terms that are either further developed or shown to be asymptotically negligible by bounding their variance with the help of the Poincaré-Nash inequality (Lemma 7 in Appendix [C). The analysis makes use of several auxiliary results summarized in Appendix C In more detail, the proof consists of the following three main steps: 
1) We first take the expectation over $W$ by fixing $\mathbf{X} \in \mathcal{S}_{=}^{n}$ and $\mathbf{H} \in \mathbb{C}^{N \times K}$ : we define the function $\phi_{n}^{\mathbf{X}^{n}, \mathbf{H}^{n}}(t) \triangleq \mathbb{E}\left[e^{\mathbf{i} t \Gamma_{n}^{\mathbf{X}^{n}, \mathbf{H}^{n}}}\right]$, where $\Gamma_{n}^{\mathbf{X}^{n}, \mathbf{H}^{n}}$ is the random variable $\Gamma_{n}$ taken for fixed $H=\mathbf{H}_{n}$ and $X=\mathbf{X}^{n}$, and show that

$$
\begin{aligned}
& \frac{\partial \phi_{n}^{\mathbf{X}^{n}, \mathbf{H}^{n}}(t)}{\partial t}= \\
& \left(\mathbf{i} \mu_{n}^{\mathbf{X}^{n}, \mathbf{H}^{n}}-t\left(\theta_{n}^{\mathbf{X}^{n}, \mathbf{H}^{n}}\right)^{2}+\mathbf{i} t^{2} \kappa_{n}^{\mathbf{X}^{n}, \mathbf{H}^{n}}\right) \phi_{n}^{\mathbf{X}^{n}, \mathbf{H}^{n}}(t) \\
& \quad+\bar{\varepsilon}_{n}^{\mathbf{X}^{n}, \mathbf{H}^{n}}(t)
\end{aligned}
$$

for some $\mu_{n}^{\mathbf{X}^{n}, \mathbf{H}^{n}}=\mathcal{O}(n), \theta_{n}^{\mathbf{X}^{n}, \mathbf{H}^{n}}=\mathcal{O}(1), \kappa_{n}^{\mathbf{X}^{n}, \mathbf{H}^{n}}=$ $\mathcal{O}\left(n^{-1}\right)$, and $\bar{\varepsilon}_{n}^{\mathbf{X}^{n}, \mathbf{H}^{n}}(t)=\mathcal{O}\left(n^{-2}\right)$ which must be carefully controlled. This establishes a differential equation for $\phi_{n}^{\mathbf{X}^{n}, \mathbf{H}^{n}}(t)$ the solution of which allows us to obtain an estimate of $\phi_{n}^{\mathbf{X}^{n}, \mathbf{H}^{n}}(t)$ under the form $e^{f(t, \mathbf{X}, \mathbf{H})}$ (i.e., with no expectation over $W)_{2}^{2}$

2) We then compute the expectation over $H$ : we introduce the function $\phi_{n}^{\mathbf{X}^{n}}(t) \triangleq \mathbb{E}\left[\phi_{n}^{\mathbf{X}^{n}, H^{n}}(t)\right]$. Working mainly with the tractable estimator $e^{f(t, \mathbf{X}, \mathbf{H})}$ of $\phi_{n}^{\mathbf{X}^{n}, \mathbf{H}^{n}}(t)$ as developed in step 1), instead of $\phi_{n}^{\mathbf{X}^{n}, \mathbf{H}^{n}}(t)$ itself, we prove in a similar fashion that

$$
\frac{\partial \phi_{n}^{\mathbf{X}^{n}}(t)}{\partial t}=\left(\mathbf{i} \mu_{n}^{\mathbf{X}^{n}}-t\left(\theta_{n}^{\mathbf{X}^{n}}\right)^{2}\right) \phi_{n}^{\mathbf{X}^{n}}(t)+\varepsilon_{n}^{\mathbf{X}^{n}}(t)
$$

for some $\mu_{n}^{\mathbf{X}^{n}}=\mathcal{O}(n), \theta_{n}^{\mathbf{X}^{n}}$, and $\varepsilon_{n}^{\mathbf{X}^{n}}(t)=\mathcal{O}\left(n^{-1}\right)$. This establishes a second differential equation.

3) We finally integrate (163) and show that

$$
\tilde{\phi}_{n}^{\mathbf{X}^{n}} \triangleq \mathbb{E}\left[e^{\mathbf{i} \frac{t}{\theta_{n}^{\mathbf{X}^{n}}}\left(\Gamma_{n}^{\mathbf{X}^{n}}-\mu_{n}^{\mathbf{X}^{n}}\right)}\right]=e^{-\frac{t^{2}}{2}}+\mathcal{O}\left(n^{-\frac{1}{2}}\right)
$$

(as $n \stackrel{(\beta, c)}{\longrightarrow} \infty$ ). Since (164) holds almost surely for any random matrix $X^{n}$ with law $\mathbb{P}_{X^{n}} \in \mathcal{P}\left(\mathcal{S}_{\overline{=}}^{n}\right)$ for all $n$, it holds also for the function $\tilde{\phi}_{n}(t)=\mathbb{E}\left[\tilde{\phi}_{n}^{X^{n}}(t)\right]=$ $\mathbb{E}\left[e^{\frac{i t}{\theta_{n}}\left(\Gamma_{n}-\mu_{n}\right)}\right]$ which finally proves $(160)$.

We now detail all these steps rigorously.

Step 1:

In a first step, we consider the expectation over $W$ by treating $\mathbf{H} \in \mathbb{C}^{N \times K}$ and $\mathbf{X} \in \mathcal{S}_{=}^{n}$ fixed. We define the function $\phi_{n}^{\mathbf{X}^{n}, \mathbf{H}^{n}}(t) \triangleq \mathbb{E}\left[e^{\mathbf{i} t \Gamma_{n}^{\mathbf{x}^{n}, \mathbf{H}^{n}}}\right]$ which we would like to express as a differential equation of the form $\frac{\partial \phi_{n}^{\mathbf{X}^{n}, \mathbf{H}^{n}}(t)}{\partial t}=$ $f(\mathbf{X}, \mathbf{H}, t) \phi_{n}^{\mathbf{X}^{n}, \mathbf{H}^{n}}(t)+\bar{\varepsilon}_{n}^{\mathbf{X}^{n}, \mathbf{H}^{n}}(t)$ for some functional $f$ and quantity $\bar{\varepsilon}_{n}^{\mathbf{X}^{n}, \mathbf{H}^{n}}(t)$ which vanishes asymptotically. Since $\Gamma_{n}^{\mathbf{X}^{n}}, \mathbf{H}^{n}$ is real, $\phi_{n}^{\mathbf{X}^{n}, \mathbf{H}^{n}}(-t)=\phi_{n}^{\mathbf{X}^{n}, \mathbf{H}^{n}}(t)^{*}$, so that it is sufficient to consider $t \geq 0$ for the rest of the proof.

${ }^{2}$ Note importantly that, although the term $\kappa_{n}^{\mathbf{X}^{n}, \mathbf{H}^{n}}$ is of order $\mathcal{O}\left(n^{-1}\right)$ and will not play a role at the end of the calculus, it needs to be isolated and not contained into $\bar{\varepsilon}_{n}^{\mathbf{X}^{n}}, \mathbf{H}^{n}(t)$ as the estimation error $\phi_{n}^{\mathbf{X}^{n}, \mathbf{H}^{n}}(t)-e^{f(t, \mathbf{X}, \mathbf{H})}$, which is of the same order of magnitude as $\bar{\varepsilon}_{n}^{\mathbf{X}^{n}, \mathbf{H}^{n}}(t)$, will increase by a factor $n$ when we take its expectation over $\mathbf{H}$ (this is due to $\mu_{n}^{\mathbf{X}^{n}, \mathbf{H}^{n}}$ being of order $\mathcal{O}(n))$.
With the help of (152), we can decompose $\Gamma_{n}$ in the following way:

$$
\Gamma_{n}=\Gamma_{n, 1}+\Gamma_{n, 2}+\Gamma_{n, 3}+\Gamma_{n, 4}
$$

where

$$
\begin{aligned}
\Gamma_{n, 1}= & \sqrt{\frac{n}{K}} \log \operatorname{det}\left(\mathbf{I}_{N}+\frac{1}{\sigma^{2}} \frac{1}{K} H H^{\mathrm{H}}\right) \\
& +\frac{1}{\sqrt{n K}} \operatorname{tr} Q \frac{H X X^{\mathrm{H}} H^{\mathrm{H}}}{K} \\
\Gamma_{n, 2}= & -\frac{1}{\sqrt{n K}} \operatorname{tr} Q \frac{H H^{\mathrm{H}}}{K} W W^{\mathrm{H}} \\
\Gamma_{n, 3}= & \frac{\sigma}{\sqrt{n K}} \operatorname{tr} Q \frac{H X W^{\mathrm{H}}}{\sqrt{K}} \\
\Gamma_{n, 4}= & \frac{\sigma}{\sqrt{n K}} \operatorname{tr} Q \frac{W X^{\mathrm{H}} H^{\mathrm{H}}}{\sqrt{K}}
\end{aligned}
$$

and where we have defined $Q \triangleq Q\left(\sigma^{2}\right)$ to simplify the notations.

By (165),

$$
\frac{\partial \phi_{n}^{\mathbf{X}^{n}, \mathbf{H}^{n}}(t)}{\partial t}=\sum_{k=1}^{4} \mathbf{i} \mathbb{E}\left[\Gamma_{n, k}^{\mathbf{X}^{n}, \mathbf{H}^{n}} e^{\mathbf{i} t \Gamma_{n}^{\mathbf{x}^{n}, \mathbf{H}^{n}}}\right] .
$$

Since $\Gamma_{n, 1}^{\mathbf{X}^{n}, \mathbf{H}^{n}}$ is independent of $W$,

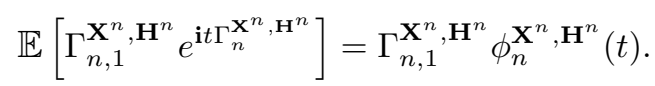

The term in $\Gamma_{n, 2}^{\mathbf{X}^{n}, \mathbf{H}^{n}}$ is studied as follows:

$$
\begin{aligned}
& \mathbb{E}\left[\Gamma_{n, 2}^{\mathbf{X}^{n}, \mathbf{H}^{n}}\right] \\
& =-\frac{1}{\sqrt{n K}} \mathbb{E}\left[\operatorname{tr} \mathbf{Q} \frac{\mathbf{H} \mathbf{H}^{\mathrm{H}}}{K} W W^{\mathrm{H}} e^{\left.\mathbf{i} t \Gamma_{n}^{\mathbf{x}^{n}, \mathbf{H}^{n}}\right]}\right. \\
& =-\frac{1}{\sqrt{n K}} \sum_{k=1}^{N} \sum_{i=1}^{N}\left[\mathbf{Q} \frac{\mathbf{H} \mathbf{H}^{\mathrm{H}}}{K}\right]_{k i} \mathbb{E}\left[\left[W W^{\mathbf{H}}\right]_{i k} e^{\left.\mathbf{i} t \Gamma_{n}^{\mathbf{X}^{n}, \mathbf{H}^{n}}\right]}\right. \\
& =-\frac{1}{\sqrt{n K}} \sum_{k=1}^{N} \sum_{i=1}^{N}\left[\mathbf{Q} \frac{\mathbf{H} \mathbf{H}^{\mathrm{H}}}{K}\right]_{k i} \sum_{j=1}^{n} \mathbb{E}\left[W_{i j} W_{k j}^{*} e^{\left.\mathbf{i} t \Gamma_{n}^{\mathbf{x}^{n}, \mathbf{H}^{n}}\right] .}\right.
\end{aligned}
$$

We now use the integration by parts formula (Lemma 6 in Appendix (D) to develop the individual terms $\mathbb{E}\left[W_{i j} W_{k j}^{*} e^{\left.\mathbf{i} t \Gamma_{n}^{\mathbf{x}^{n}, \mathbf{H}^{n}}\right]}\right.$ as follows:

$$
\begin{aligned}
& \mathbb{E}\left[W_{i j} W_{k j}^{*} e^{\mathbf{i} t \Gamma_{n}^{\mathbf{X}^{n}, \mathbf{H}^{n}}}\right] \\
& =\delta_{i k} \mathbb{E}\left[e^{\mathbf{i} t \Gamma_{n}^{\mathbf{X}^{n}, \mathbf{H}^{n}}}\right]+\mathbf{i} t \mathbb{E}\left[W_{k j}^{*} \frac{\partial \Gamma_{n}^{\mathbf{X}^{n}, \mathbf{H}^{n}}}{\partial W_{i j}^{*}} e^{\mathbf{i} t \Gamma_{n}^{\mathbf{X}^{n}, \mathbf{H}^{n}}}\right] .
\end{aligned}
$$

The derivatives $\frac{\partial \Gamma_{n, k}^{\mathbf{X}^{n}, \mathbf{H}^{n}}}{\partial W_{i j}^{*}}$ and $\frac{\partial \Gamma_{n, k}^{\mathbf{X}^{n}, \mathbf{H}^{n}}}{\partial W_{i j}}$ can be computed by straightforward application of the derivation rules provided in 
Lemma 8 in Appendix C:

$$
\begin{aligned}
& \frac{\partial \Gamma_{n, 1}}{\partial W_{i j}^{*}}=\frac{\partial \Gamma_{n, 1}}{\partial W_{i j}}=\frac{\partial \Gamma_{n, 3}}{\partial W_{i j}}=\frac{\partial \Gamma_{n, 4}}{\partial W_{i j}^{*}}=0 \\
& \frac{\partial \Gamma_{n, 2}}{\partial W_{i j}^{*}}=-\frac{1}{\sqrt{n K}}\left[Q \frac{H H^{\mathrm{H}}}{K} W\right]_{i j} \\
& \frac{\partial \Gamma_{n, 2}}{\partial W_{i j}}=-\frac{1}{\sqrt{n K}}\left[W^{\mathrm{H}} \frac{H H^{\mathrm{H}}}{K} Q\right]_{j i} \\
& \frac{\partial \Gamma_{n, 3}}{\partial W_{i j}^{*}}=\frac{\sigma}{\sqrt{n K}}\left[Q \frac{H}{\sqrt{K}} X\right]_{i j} \\
& \frac{\partial \Gamma_{n, 4}}{\partial W_{i j}}=\frac{\sigma}{\sqrt{n K}}\left[X^{\mathrm{H}} \frac{H^{\mathrm{H}}}{\sqrt{K}} Q\right]_{j i} .
\end{aligned}
$$

Using (165) together with the derivatives 176, 177, 179 in 175, we obtain

$$
\begin{aligned}
& \mathbb{E}\left[\left[W W^{\mathbf{H}}\right]_{i k} e^{\left.\mathbf{i} t \Gamma_{n}^{\mathbf{X}^{n}, \mathbf{H}^{n}}\right]}\right. \\
& =n \delta_{i k} \phi_{n}^{\mathbf{X}^{n}, \mathbf{H}^{n}}(t) \\
& \quad+\mathbf{i} t \sum_{j=1}^{n} \mathbb{E}\left[W_{k j}^{*}\left(\frac{\partial \Gamma_{n, 2}^{\mathbf{X}^{n}, \mathbf{H}^{n}}}{\partial W_{i j}^{*}}+\frac{\partial \Gamma_{n, 3}^{\mathbf{X}^{n}, \mathbf{H}^{n}}}{\partial W_{i j}^{*}}\right) e^{\left.\mathbf{i} t \Gamma_{n}^{\mathbf{X}^{n}, \mathbf{H}^{n}}\right]}\right. \\
& =n \delta_{i k} \phi_{n}^{\mathbf{X}^{n}, \mathbf{H}^{n}}(t)-\mathbf{i} t \frac{1}{\sqrt{n K}} \mathbb{E}\left[\left(\left[\mathbf{Q} \frac{\mathbf{H} \mathbf{H}^{\mathbf{H}}}{K} W W^{\mathbf{H}}\right]_{i k}\right.\right. \\
& \left.\quad-\sigma\left[\mathbf{Q} \frac{\mathbf{H}}{\sqrt{K}} \mathbf{X} W^{\mathbf{H}}\right]_{i k}\right) e^{\left.\mathbf{i} t \Gamma_{n}^{\mathbf{X}^{n}, \mathbf{H}^{n}}\right] .}
\end{aligned}
$$

Replacing the last result in 173 yields

$$
\begin{aligned}
\mathbb{E} & {\left[\Gamma_{n, 2}^{\mathbf{X}^{n}, \mathbf{H}^{n}} e^{\left.\mathbf{i} t \Gamma_{n}^{\mathbf{X}^{n}, \mathbf{H}^{n}}\right]}\right.} \\
= & -\frac{n}{\sqrt{n K}} \operatorname{tr} \mathbf{Q} \frac{\mathbf{H} \mathbf{H}^{\mathbf{H}}}{K} \phi_{n}^{\mathbf{X}^{n}, \mathbf{H}^{n}}(t) \\
& +\mathbf{i} t \mathbb{E}\left[\left(\frac{1}{n K} \operatorname{tr}\left(\mathbf{Q} \frac{\mathbf{H} \mathbf{H}^{\mathbf{H}}}{K}\right)^{2} W W^{\mathbf{H}}\right.\right. \\
& \left.\quad-\frac{\sigma}{n K} \operatorname{tr} \mathbf{Q} \frac{\mathbf{H} \mathbf{H}^{\mathbf{H}}}{K} \mathbf{Q} \frac{\mathbf{H}}{\sqrt{K}} \mathbf{X} W^{\mathbf{H}}\right) e^{\left.\mathbf{i} t \Gamma_{n}^{\mathbf{X}^{n}, \mathbf{H}^{n}}\right] .}
\end{aligned}
$$

We will now individually treat the second and third terms on the RHS of the last equation. For the second term, using the same steps as above, we arrive at

$$
\begin{aligned}
& \mathbb{E}\left[\frac{1}{n K} \operatorname{tr}\left(\mathbf{Q} \frac{\mathbf{H H}^{\mathrm{H}}}{K}\right)^{2} W W^{\mathrm{H}} e^{\mathbf{i} t \Gamma_{n}^{\mathbf{X}^{n}, \mathbf{H}^{n}}}\right] \\
& =\frac{1}{n K} \sum_{k=1}^{N} \sum_{i=1}^{N}\left[\left(\mathbf{Q} \frac{\mathbf{H} \mathbf{H}^{\mathbf{H}}}{K}\right)^{2}\right]_{k i} \mathbb{E}\left[\left[W W^{\mathbf{H}}\right]_{i k} e^{\mathbf{i} t \Gamma_{n}^{\mathbf{X}^{n}, \mathbf{H}^{n}}}\right] \\
& =\frac{n}{n K} \operatorname{tr}\left(\mathbf{Q} \frac{\mathbf{H} \mathbf{H}^{\mathbf{H}}}{K}\right)^{2} \phi_{n}^{\mathbf{X}^{n}, \mathbf{H}^{n}}(t) \\
& -\frac{\mathbf{i} t}{(n K)^{\frac{3}{2}}} \mathbb{E}\left[\left(\operatorname{tr}\left(\mathbf{Q} \frac{\mathbf{H} \mathbf{H}^{\mathrm{H}}}{K}\right)^{3} W W^{\mathrm{H}}\right.\right. \\
& \left.\left.-\sigma \operatorname{tr}\left(\mathbf{Q} \frac{\mathbf{H} \mathbf{H}^{\mathrm{H}}}{K}\right)^{2} \mathbf{Q} \frac{\mathbf{H}}{\sqrt{K}} \mathbf{X} W^{\mathbf{H}}\right) e^{\mathbf{i} t \Gamma_{n}^{\mathbf{X}^{n}, \mathbf{H}^{n}}}\right] \\
& =\frac{n}{n K} \operatorname{tr}\left(\mathbf{Q} \frac{\mathbf{H H}^{\mathrm{H}}}{K}\right)^{2} \phi_{n}^{\mathbf{X}^{n}, \mathbf{H}^{n}}(t) \\
& -\mathbf{i} t \frac{n}{\sqrt{n^{3} K^{3}}} \operatorname{tr}\left(\mathbf{Q} \frac{\mathbf{H} \mathbf{H}^{\mathbf{H}}}{K}\right)^{3} \phi_{n}^{\mathbf{X}^{n}, \mathbf{H}^{n}}(t)+\varepsilon_{n, 1}^{\mathbf{X}^{n}, \mathbf{H}^{n}}(t)
\end{aligned}
$$

where

$$
\begin{aligned}
& \varepsilon_{n, 1}^{\mathbf{X}^{n}, \mathbf{H}^{n}}(t)= \\
& -\mathbf{i} t \mathbb{E}\left[\frac{n}{\sqrt{n^{3} K^{3}}} \operatorname{tr}\left(\mathbf{Q} \frac{\mathbf{H} \mathbf{H}^{\mathbf{H}}}{K}\right)^{3}\left(\frac{W W^{\mathbf{H}}}{n}-\mathbf{I}_{N}\right) e^{\mathbf{i} t \Gamma_{n}^{\mathbf{X}^{n}, \mathbf{H}^{n}}}\right] \\
& +\mathbf{i} t \mathbb{E}\left[\frac{\sigma}{(n K)^{\frac{3}{2}}} \operatorname{tr} \mathbf{Q}\left(\mathbf{Q} \frac{\mathbf{H} \mathbf{H}^{\mathbf{H}}}{K}\right)^{2} \frac{\mathbf{H}}{\sqrt{K}} \mathbf{X} W^{\mathbf{H}} e^{\left.\mathbf{i} t \Gamma_{n}^{\mathbf{X}^{n}, \mathbf{H}^{n}}\right] .}\right.
\end{aligned}
$$

Consider now the third term on the RHS of (183) and define $\mathbf{T}=\mathbf{Q} \frac{\mathbf{H H}^{\mathrm{H}}}{K} \mathbf{Q} \frac{\mathbf{H}}{\sqrt{K}} \mathbf{X}$. Then,

$$
\begin{aligned}
& \frac{\sigma}{n K} \mathbb{E}\left[\operatorname{tr} \mathbf{T} W^{\mathrm{H}} e^{\mathbf{i} t \Gamma_{n}^{\mathbf{X}^{n}, \mathbf{H}^{n}}}\right] \\
& =\mathbf{i} t \frac{\sigma}{n K} \sum_{i=1}^{N} \sum_{j=1}^{n} T_{i j} \mathbb{E}\left[\frac{\partial \Gamma_{n}^{\mathbf{X}^{n}, \mathbf{H}^{n}}}{\partial W_{i j}} e^{\left.\mathbf{i} t \Gamma_{n}^{\mathbf{X}^{n}, \mathbf{H}^{n}}\right]}\right. \\
& =\mathbf{i} t \frac{\sigma^{2}}{\sqrt{n^{3} K^{3}}} \operatorname{tr} \mathbf{Q}^{2} \frac{\mathbf{H} \mathbf{H}^{\mathrm{H}}}{K} \mathbf{Q} \frac{\mathbf{H X X} \mathbf{X}^{\mathbf{H}} \mathbf{H}^{\mathbf{H}}}{K} \phi_{n}^{\mathbf{X}^{n}, \mathbf{H}^{n}}(t) \\
& +\varepsilon_{n, 2}^{\mathbf{X}^{n}, \mathbf{H}^{n}}(t)
\end{aligned}
$$

where

$$
\begin{aligned}
& \varepsilon_{n, 2}^{\mathbf{X}^{n}, \mathbf{H}^{n}}(t)= \\
& -\mathbf{i} t \frac{\sigma}{(n K)^{\frac{3}{2}}} \mathbb{E}\left[\operatorname{tr} \mathbf{Q}\left(\mathbf{Q} \frac{\mathbf{H} \mathbf{H}^{\mathbf{H}}}{\sqrt{K}}\right)^{2} \frac{\mathbf{H}}{\sqrt{K}} \mathbf{X} W^{\mathbf{H}} e^{\mathbf{i} t \Gamma_{n}^{\mathbf{X}^{n}, \mathbf{H}^{n}}}\right] .
\end{aligned}
$$


Combining the last results, we arrive at

$$
\begin{aligned}
& \mathbb{E}\left[\Gamma_{n, 2}^{\mathbf{X}^{n}, \mathbf{H}^{n}} e^{\left.\mathbf{i} t \Gamma_{n}^{\mathbf{X}^{n}, \mathbf{H}^{n}}\right]=}\right. \\
& -\frac{n}{\sqrt{n K}} \operatorname{tr} \mathbf{Q} \frac{\mathbf{H} \mathbf{H}^{\mathbf{H}}}{K} \phi_{n}^{\mathbf{X}^{n}, \mathbf{H}^{n}}(t) \\
& +\mathbf{i} t \frac{n}{n K} \operatorname{tr}\left(\mathbf{Q} \frac{\mathbf{H} \mathbf{H}^{\mathbf{H}}}{K}\right)^{2} \phi_{n}^{\mathbf{X}^{n}, \mathbf{H}^{n}(t)} \\
& +t^{2}\left\{\frac{n}{\sqrt{n^{3} K^{3}}} \operatorname{tr}\left(\mathbf{Q} \frac{\mathbf{H} \mathbf{H}^{\mathbf{H}}}{K}\right)^{3}\right. \\
& \left.+\frac{\sigma^{2} n}{\sqrt{n^{3} K^{3}}} \operatorname{tr} \mathbf{Q}^{2} \frac{\mathbf{H} \mathbf{H}^{\mathbf{H}}}{K} \mathbf{Q} \frac{\mathbf{H X X} \mathbf{X}^{\mathbf{H}} \mathbf{H}^{\mathbf{H}}}{n K}\right\} \phi_{n}^{\mathbf{X}^{n}, \mathbf{H}^{n}}(t) \\
& +\mathbf{i} t\left\{\varepsilon_{n, 1}^{\mathbf{X}^{n}, \mathbf{H}^{n}}(t)-\varepsilon_{n, 2}^{\mathbf{X}^{n}, \mathbf{H}^{n}}(t)\right\} .
\end{aligned}
$$

We now consider the terms in $\Gamma_{n, 4}^{\mathbf{X}^{n}, \mathbf{H}^{n}}$ and $\Gamma_{n, 3}^{\mathbf{X}^{n}, \mathbf{H}^{n}}$. Using similar calculus as above,

$$
\begin{aligned}
& \mathbb{E}\left[\Gamma_{n, 4}^{\mathbf{X}^{n}, \mathbf{H}^{n}} e^{i t \Gamma_{n}^{\mathbf{X}^{n}, \mathbf{H}^{n}}}\right] \\
& =\mathbb{E}\left[\frac{\sigma}{\sqrt{n K}} \operatorname{tr} \mathbf{Q} W \mathbf{X}^{\mathrm{H}} \frac{\mathbf{H}^{\mathrm{H}}}{\sqrt{K}} e^{\mathbf{i} t \Gamma_{n}^{\mathbf{x}^{n}, \mathbf{H}^{n}}}\right] \\
& =\frac{\sigma}{\sqrt{n K}} \sum_{i=1}^{N} \sum_{j=1}^{n}\left[\mathbf{X}^{\mathrm{H}} \frac{\mathbf{H}^{\mathrm{H}}}{\sqrt{K}} \mathbf{Q}\right]_{j i} \mathbb{E}\left[W_{i j} e^{\mathbf{i} t \Gamma_{n}^{\mathbf{x}^{n}, \mathbf{H}^{n}}}\right] \\
& =\mathbf{i} t \frac{\sigma}{\sqrt{n K}} \sum_{i=1}^{N} \sum_{j=1}^{n}\left[\mathbf{X}^{\mathrm{H}} \frac{\mathbf{H}^{\mathrm{H}}}{\sqrt{K}} \mathbf{Q}\right]_{j i} \mathbb{E}\left[\frac{\partial \Gamma_{n}^{\mathbf{X}^{n}, \mathbf{H}^{n}}}{\partial W_{i j}^{*}} e^{\mathrm{i} t \Gamma_{n}^{\mathbf{X}^{n}, \mathbf{H}^{n}}}\right] \\
& =\mathbf{i} t \frac{\sigma}{\sqrt{n K}} \sum_{i=1}^{N} \sum_{j=1}^{n}\left[\mathbf{X}^{\mathrm{H}} \frac{\mathbf{H}^{\mathrm{H}}}{\sqrt{K}} \mathbf{Q}\right]_{j i} \mathbb{E}\left[\left(\frac{\sigma}{\sqrt{n K}}\left[\mathbf{Q} \frac{\mathbf{H}}{\sqrt{K}} \mathbf{X}\right]_{i j}\right.\right. \\
& \left.\left.-\frac{1}{\sqrt{n K}}\left[\mathbf{Q} \frac{\mathbf{H H}^{\mathbf{H}}}{K} W\right]_{i j}\right) e^{\mathbf{i} t \Gamma_{n}^{\mathbf{X}^{n}, \mathbf{H}^{n}}}\right] \\
& =\mathbf{i} t \frac{\sigma^{2} n}{n K} \operatorname{tr} \mathbf{Q}^{2} \frac{\mathbf{H X X} \mathbf{X}^{\mathrm{H}} \mathbf{H}^{\mathrm{H}}}{(n+1) K} \phi_{n}^{\mathbf{X}^{n}, \mathbf{H}^{n}}(t) \\
& -\mathbf{i} t \mathbb{E}\left[\frac{\sigma}{n K} \operatorname{tr} \mathbf{Q} \frac{\mathbf{H} \mathbf{H}^{\mathrm{H}}}{K} W \mathbf{X}^{\mathrm{H}} \frac{\mathbf{H}^{\mathrm{H}}}{\sqrt{K}} \mathbf{Q} e^{\mathbf{i} t \Gamma_{n}^{\mathbf{x}^{n}, \mathbf{H}^{n}}}\right] .
\end{aligned}
$$

Doing the same calculus for the second term on the RHS of the last equation, one arrives at

$$
\begin{aligned}
\mathbb{E} & {\left[\frac{\sigma}{n K} \operatorname{tr} \mathbf{Q} \frac{\mathbf{H} \mathbf{H}^{\mathrm{H}}}{K} W \mathbf{X}^{\mathrm{H}} \frac{\mathbf{H}^{\mathrm{H}}}{\sqrt{K}} \mathbf{Q} e^{\mathbf{i} t \Gamma_{n}^{\mathbf{X}^{n}, \mathbf{H}^{n}}}\right] } \\
= & \mathbf{i} t \frac{\sigma^{2} n}{\sqrt{n^{3} K^{3}}} \operatorname{tr} \mathbf{Q}^{2} \frac{\mathbf{H} \mathbf{H}^{\mathrm{H}}}{K} \mathbf{Q} \frac{\mathbf{H X X} \mathbf{X}^{\mathrm{H}} \mathbf{H}^{\mathrm{H}}}{n K} \phi_{n}^{\mathbf{X}^{n}, \mathbf{H}^{n}}(t) \\
& +\varepsilon_{n, 4}^{\mathbf{X}^{n}, \mathbf{H}^{n}}(t)
\end{aligned}
$$

where

$$
\begin{aligned}
& \varepsilon_{n, 4}^{\mathbf{X}^{n}, \mathbf{H}^{n}}(t)= \\
& -\mathbf{i} t \frac{\sigma}{(n K)^{\frac{3}{2}}} \mathbb{E}\left[\operatorname{tr} \mathbf{Q}\left(\mathbf{Q} \frac{\mathbf{H} \mathbf{H}^{\mathbf{H}}}{K}\right)^{2} W \mathbf{X}^{\mathbf{H}} \frac{\mathbf{H}^{\mathbf{H}}}{\sqrt{K}} e^{\mathbf{i} t \Gamma_{n}^{\mathbf{X}^{n}, \mathbf{H}^{n}}}\right] .
\end{aligned}
$$

Thus,

$$
\begin{aligned}
\mathbb{E} & {\left[\Gamma_{n, 4}^{\mathbf{X}^{n}, \mathbf{H}^{n}} e^{\left.i t \Gamma_{n}^{\mathbf{X}^{n}, \mathbf{H}^{n}}\right]}\right.} \\
= & \mathbf{i} t \frac{\sigma^{2} n}{n K} \operatorname{tr} \mathbf{Q}^{2} \frac{\mathbf{H X X} \mathbf{X}^{\mathrm{H}} \mathbf{H}^{\mathbf{H}}}{n K} \phi_{n}^{\mathbf{X}^{n}, \mathbf{H}^{n}}(t) \\
& +t^{2} \frac{\sigma^{2} n}{\sqrt{n^{3} K^{3}}} \operatorname{tr} \mathbf{Q}^{2} \frac{\mathbf{H} \mathbf{H}^{\mathrm{H}}}{K} \mathbf{Q} \frac{\mathbf{H X X} \mathbf{X}^{\mathbf{H}} \mathbf{H}^{\mathbf{H}}}{n K} \phi_{n}^{\mathbf{X}^{n}, \mathbf{H}^{n}}(t) \\
& -\mathbf{i} t \varepsilon_{n, 4}^{\mathbf{X}^{n}, \mathbf{H}^{n}}(t) .
\end{aligned}
$$

Since $\Gamma_{n, 3}^{\mathbf{X}^{n}, \mathbf{H}^{n}}=\left(\Gamma_{n, 4}^{\mathbf{X}^{n}, \mathbf{H}^{n}}\right)^{*}$, it follows that

$$
\mathbb{E}\left[\Gamma_{n, 3}^{\mathbf{X}^{n}, \mathbf{H}^{n}} e^{i t \Gamma_{n}^{\mathbf{X}^{n}, \mathbf{H}^{n}}}\right]=\mathbb{E}\left[\Gamma_{n, 4}^{\mathbf{X}^{n}, \mathbf{H}^{n}} e^{-i t \Gamma_{n}^{\mathbf{X}^{n}, \mathbf{H}^{n}}}\right]^{*} .
$$

Gathering all pieces together as a polynomial in $t$, we obtain a first differential equation of $\phi_{n}^{\mathbf{X}^{n}, \mathbf{H}^{n}}(t)$ as given in (202)(206) on the top of the next page, where

$$
\mathbf{A} \triangleq \mathbf{I}_{K}-\frac{1}{n} \mathbf{X X}^{\mathbf{H}}
$$

Let us now have a closer look at the quantities $\theta_{n}^{\mathbf{X}^{n}, \mathbf{H}^{n}}$, $\kappa_{n}^{\mathbf{X}^{n}, \mathbf{H}^{n}}$, and $\bar{\varepsilon}_{n}^{\mathbf{X}^{n}, \mathbf{H}^{n}}(t)$ individually. Using the identities and bounds presented at the beginning of this proof, one can verify that

$$
\begin{aligned}
0 \leq\left(\theta_{n}^{\mathbf{X}^{n}, \mathbf{H}^{n}}\right)^{2} & \leq \frac{N}{K}+\frac{2}{n K} \operatorname{tr} \mathbf{X} \mathbf{X}^{\mathbf{H}}=\left(\frac{N}{K}+2\right) \\
0 \leq \kappa_{n}^{\mathbf{X}^{n}, \mathbf{H}^{n}} & \leq \frac{N}{\sqrt{n K^{3}}}+\frac{3}{\sqrt{n^{3} K^{3}}} \operatorname{tr} \mathbf{X X}^{\mathbf{H}} \\
& =\frac{1}{\sqrt{n K}}\left(\frac{N}{K}+3\right) .
\end{aligned}
$$

Based on Remark 7 in Appendix C, we can bound the absolute value of $\bar{\varepsilon}_{n}^{\mathbf{X}^{n}, \mathbf{H}^{n}}(t)$ as

$$
\begin{aligned}
& \left|\bar{\varepsilon}_{n}^{\mathbf{X}^{n}, \mathbf{H}^{n}}(t)\right| \leq \\
& t^{3}\left(\sqrt{\operatorname{Var}\left[\frac{1}{\sqrt{n K^{3}}} \operatorname{tr}\left(\mathbf{Q} \frac{\mathbf{H} \mathbf{H}^{\mathbf{H}}}{K}\right)^{3}\left(\frac{W W^{\mathbf{H}}}{n}-\mathbf{I}_{N}\right)\right]}\right. \\
& +4 \sqrt{\left.\operatorname{Var}\left[\frac{\sigma}{\sqrt{n^{3} K^{3}}} \operatorname{tr} \mathbf{Q}\left(\mathbf{Q} \frac{\mathbf{H} \mathbf{H}^{\mathbf{H}}}{K}\right)^{2} \frac{\mathbf{H X} W^{\mathbf{H}}}{\sqrt{K}}\right]\right)} .
\end{aligned}
$$

By Lemma 10 (ii) in Appendix E-A it follows that

$$
\begin{aligned}
& \operatorname{Var}\left[\frac{1}{\sqrt{n K^{3}}} \operatorname{tr}\left(\mathbf{Q} \frac{\mathbf{H} \mathbf{H}^{\mathrm{H}}}{K}\right)^{3}\left(\frac{W W^{\mathrm{H}}}{n}-\mathbf{I}_{N}\right)\right] \\
& =\operatorname{Var}\left[\frac{n}{\sqrt{n^{3} K^{3}}} \operatorname{tr}\left(\mathbf{Q} \frac{\mathbf{H} \mathbf{H}^{\mathrm{H}}}{K}\right)^{3} \frac{W W^{\mathrm{H}}}{n}\right] \\
& \leq \frac{2}{n K^{3}} \operatorname{tr}\left(\mathbf{Q} \frac{\mathbf{H H}}{K}\right)^{\mathrm{H}} \\
& \leq \frac{2 N}{n K^{3}} .
\end{aligned}
$$




$$
\begin{aligned}
& \frac{\partial \phi_{n}^{\mathbf{X}^{n}, \mathbf{H}^{n}}(t)}{\partial t}=\left(\mathbf{i} \mu_{n}^{\mathbf{X}^{n}, \mathbf{H}^{n}}-t\left(\theta_{n}^{\mathbf{X}^{n}, \mathbf{H}^{n}}\right)^{2}+\mathbf{i} t^{2} \kappa_{n}^{\mathbf{X}^{n}, \mathbf{H}^{n}}\right) \phi_{n}^{\mathbf{X}^{n}, \mathbf{H}^{n}}(t)+\bar{\varepsilon}_{n}^{\mathbf{X}^{n}, \mathbf{H}^{n}}(t) \\
& \mu_{n}^{\mathbf{X}^{n}, \mathbf{H}^{n}}=\sqrt{\frac{n}{K}} \log \operatorname{det}\left(\mathbf{I}_{N}+\frac{1}{\sigma^{2}} \frac{\mathbf{H} \mathbf{H}^{\mathrm{H}}}{K}\right)-\frac{n}{\sqrt{n K}} \operatorname{tr} \mathbf{Q} \frac{\mathbf{H} \mathbf{A} \mathbf{H}^{\mathrm{H}}}{K} \\
& \left(\theta_{n}^{\mathbf{X}^{n}, \mathbf{H}^{n}}\right)^{2}=\frac{n}{n K} \operatorname{tr}\left(\mathbf{Q} \frac{\mathbf{H} \mathbf{H}^{\mathrm{H}}}{K}\right)^{2}+\frac{2 \sigma^{2} n}{n K} \operatorname{tr} \mathbf{Q}^{2} \frac{\mathbf{H} \mathbf{X} \mathbf{X}^{\mathrm{H}} \mathbf{H}^{\mathrm{H}}}{n K} \\
& \kappa_{n}^{\mathbf{X}^{n}, \mathbf{H}^{n}}=\frac{n}{\sqrt{n^{3} K^{3}}} \operatorname{tr}\left(\mathbf{Q} \frac{\mathbf{H} \mathbf{H}^{\mathrm{H}}}{K}\right)^{3}+\frac{3 \sigma^{2} n}{\sqrt{n^{3} K^{3}}} \operatorname{tr} \mathbf{Q}^{2} \frac{\mathbf{H H}^{\mathrm{H}}}{K} \mathbf{Q} \frac{\mathbf{H X X} \mathbf{X}^{\mathrm{H}} \mathbf{H}^{\mathrm{H}}}{n K} \\
& \bar{\varepsilon}_{n}^{\mathbf{X}^{n}, \mathbf{H}^{n}}(t)=\mathbf{i} t^{2} \mathbb{E}\left[\left\{\frac{n}{\sqrt{n^{3} K^{3}}} \operatorname{tr}\left(\mathbf{Q} \frac{\mathbf{H} \mathbf{H}^{\mathrm{H}}}{K}\right)^{3}\left(\frac{W W^{\mathrm{H}}}{n}-\mathbf{I}_{N}\right)-\frac{3 \sigma}{\sqrt{n^{3} K^{3}}} \operatorname{tr} \mathbf{Q}\left(\mathbf{Q} \frac{\mathbf{H} \mathbf{H}^{\mathrm{H}}}{K}\right)^{2} \frac{\mathbf{H X} W^{\mathbf{H}}}{\sqrt{K}}\right.\right.
\end{aligned}
$$

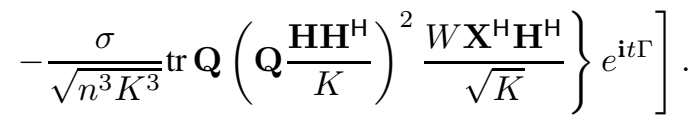

Similarly, by Lemma $10(i)$ in Appendix E-A it follows that

$$
\begin{aligned}
& \operatorname{Var}\left[\frac{\sigma}{\sqrt{n^{3} K^{3}}} \operatorname{tr} \mathbf{Q}\left(\mathbf{Q} \frac{\mathbf{H} \mathbf{H}^{\mathrm{H}}}{K}\right)^{2} \frac{\mathbf{H X} W^{\mathrm{H}}}{\sqrt{K}}\right] \\
& =\frac{\sigma^{2}}{n^{3} K^{3}} \operatorname{tr}\left(\mathbf{Q} \frac{\mathbf{H} \mathbf{H}^{\mathrm{H}}}{K}\right) \mathbf{Q} \frac{\mathbf{H X X} \mathbf{X}^{\mathrm{H}} \mathbf{H}^{\mathrm{H}}}{K} \mathbf{Q} \\
& \leq \frac{\sigma^{2}}{n^{3} K^{3}} \operatorname{tr} \tilde{\mathbf{Q}}^{2} \frac{\mathbf{H}^{\mathrm{H}} \mathbf{H}}{K} \mathbf{X} \mathbf{X}^{\mathrm{H}} \\
& \leq \frac{1}{n^{3} K^{3}} \operatorname{tr} \mathbf{X} \mathbf{X}^{\mathrm{H}} \\
& =\frac{1}{n^{2} K^{2}} .
\end{aligned}
$$

Replacing (212) and (216) in (209), we then obtain

$$
\bar{\varepsilon}_{n}^{\mathbf{X}^{n}, \mathbf{H}^{n}}(t)=\mathcal{O}\left(t^{3} n^{-2}\right) .
$$

Similarly, from 207) and 208,

$$
\begin{aligned}
\left(\theta_{n}^{\mathbf{X}^{n}, \mathbf{H}^{n}}\right)^{2} & =\mathcal{O}(1) \\
\kappa_{n}^{\mathbf{X}^{n}, \mathbf{H}^{n}} & =\mathcal{O}\left(n^{-1}\right) .
\end{aligned}
$$

Two remarks are important at this point. First observe that the introduction of $\kappa_{n}^{\mathbf{X}^{n}, \mathbf{H}^{n}}$ allows one to gain at each step one order of precision on the estimation of $\phi_{n}^{\mathbf{X}^{n}, \mathbf{H}^{n}}$ (through refinements of the coefficients of its differential equation). The choice of the order to be used is mainly ruled by the subsequent averaging steps. For the present proof, we need the error (given by $\left.\bar{\varepsilon}_{n}^{\mathbf{X}^{n}, \mathbf{H}^{n}}(t)\right)$ to be within $\mathcal{O}\left(n^{-2}\right)$.

Second, it is very important to keep the terms in $t$ in the various bounds derived here and below. The reason for this

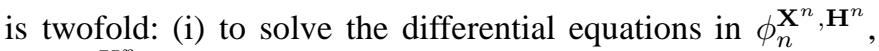
then $\phi_{n}^{\mathbf{X}^{n}}$, it will be necessary to integrate these bounds and their integrability must be controlled, (ii) at the end of the calculus, the normalization of $\Gamma_{n}$ by (the estimate for) its standard deviation $\theta_{n}^{\mathbf{X}^{n}}$, used to ensure a limiting unit variance, will be performed via a change of variable $t \mapsto t / \theta_{n}^{\mathbf{X}^{n}}$ which requires a close inspection of the polynomials in $t$ and $n^{-1}$ in the bounds.

\section{Step 2:}

In this step, we first solve (202) to express $\phi_{n}^{\mathbf{X}^{n}, \mathbf{H}^{n}}(t)$ as a function of $\mathbf{X}$ and $\mathbf{H}$. We then proceed similar to Step 1 and express the function $\phi_{n}^{\mathbf{X}^{n}}(t)=\mathbb{E}\left[\phi_{n}^{\mathbf{X}^{n}, H^{n}}(t)\right]$ as the solution of a differential equation.

The solution of 202 reads

$$
\begin{gathered}
\phi_{n}^{\mathbf{X}^{n}, \mathbf{H}^{n}}(t)=e^{\mathbf{i} t \mu_{n}^{\mathbf{X}^{n}, \mathbf{H}^{n}}-\frac{t^{2}}{2}\left(\theta_{n}^{\mathbf{X}^{n}, \mathbf{H}^{n}}\right)^{2}+\mathbf{i} \frac{t^{3}}{3} \kappa_{n}^{\mathbf{X}^{n}, \mathbf{H}^{n}}}(1+ \\
\int_{0}^{t} e^{\left.-\mathbf{i} x \mu_{n}^{\mathbf{X}^{n}, \mathbf{H}^{n}}+\frac{x^{2}}{2}\left(\theta_{n}^{\mathbf{X}^{n}, \mathbf{H}^{n}}\right)^{2}-\mathbf{i} \frac{x^{3}}{3} \kappa_{n}^{\mathbf{X}^{n}, \mathbf{H}^{n}} \bar{\varepsilon}_{n}^{\mathbf{X}^{n}, \mathbf{H}^{n}}(x) d x\right) .}
\end{gathered}
$$

Define the function $\phi_{n}^{\mathbf{X}^{n}}(t)=\mathbb{E}\left[\phi_{n}^{\mathbf{X}^{n}, H^{n}}(t)\right]$. The equation (222) on the top of the next page follows then from (220).

We will now show that only the first term on the RHS of (222) is asymptotically non-negligible. Let us first define

$$
\begin{aligned}
& \Theta= \\
& \left(\int_{0}^{t} e^{-\mathbf{i} x \mu_{n}^{\mathbf{X}^{n}, H^{n}}+\frac{x^{2}}{2}\left(\theta_{n}^{\mathbf{X}^{n}, H^{n}}\right)^{2}-\mathbf{i} \frac{x^{3}}{3} \kappa_{n}^{\mathbf{X}^{n}, H^{n}}} \bar{\varepsilon}_{n}^{\mathbf{X}^{n}, H^{n}}(x) d x\right) \\
& \quad \times e^{\mathbf{i} t \mu_{n}^{\mathbf{X}^{n}, H^{n}}-\frac{t^{2}}{2}\left(\theta_{n}^{\mathbf{X}^{n}, H^{n}}\right)^{2}+\mathbf{i} \frac{t^{3}}{3} \kappa_{n}^{\mathbf{X}^{n}, H^{n}}} .
\end{aligned}
$$

Since

$$
\begin{aligned}
|\Theta| & \leq e^{-\frac{t^{2}}{2}\left(\theta_{n}^{\mathbf{X}^{n}, H^{n}}\right)^{2}} \int_{0}^{t} e^{\frac{x^{2}}{2}\left(\theta_{n}^{\mathbf{X}^{n}, H^{n}}\right)^{2}}\left|\bar{\varepsilon}_{n}^{\mathbf{X}^{n}, H^{n}}(x)\right| d x \\
& =\mathcal{O}\left(t^{4} n^{-2}\right)
\end{aligned}
$$

it follows that $\mathbb{E}[\Theta]=\mathcal{O}\left(t^{4} n^{-2}\right)$ and $\operatorname{Var}[\Theta]=\mathcal{O}\left(t^{8} n^{-4}\right)$. Thus, by Remark 7 in Appendix $\mathrm{C}$

$$
\begin{aligned}
& \left|\mathbb{E}\left[\mu_{n}^{\mathbf{X}^{n}, H^{n} \Theta}\right]\right| \\
& \leq\left|\mathbb{E}\left[\mu_{n}^{\mathbf{X}^{n}, H^{n}}\right]\right||\mathbb{E}[\Theta]|+\sqrt{\operatorname{Var}\left[\mu_{n}^{\mathbf{X}^{n}, H^{n}}\right]} \sqrt{\operatorname{Var}[\Theta]} \\
& \leq\left|\mathbb{E}\left[\mu_{n}^{\mathbf{X}^{n}, H^{n}}\right]\right| \mathcal{O}\left(t^{4} n^{-2}\right)+\sqrt{\operatorname{Var}\left[\mu_{n}^{\mathbf{X}^{n}, H^{n}}\right]} \mathcal{O}\left(t^{4} n^{-2}\right) .
\end{aligned}
$$




$$
\begin{aligned}
& \frac{\partial \phi_{n}^{\mathbf{X}^{n}}(t)}{\partial t}=\mathbb{E}\left[\frac{\partial \phi_{n}^{\mathbf{X}^{n}, H^{n}}(t)}{\partial t}\right] \\
& =\mathbb{E}\left[\left(\mathbf{i} \mu_{n}^{\mathbf{X}^{n}, H^{n}}-t\left(\theta_{n}^{\mathbf{X}^{n}, H^{n}}\right)^{2}+\mathbf{i} t^{2} \kappa_{n}^{\mathbf{X}^{n}, H^{n}}\right) e^{\mathbf{i} t \mu_{n}^{\mathbf{X}^{n}, H^{n}}-\frac{t^{2}}{2}\left(\theta_{n}^{\mathbf{X}^{n}, H^{n}}\right)^{2}+\mathbf{i} \frac{t^{3}}{3} \kappa_{n}^{\mathbf{X}^{n}, H^{n}}}\right] \\
& +\mathbb{E}\left[\left(\mathbf{i} \mu_{n}^{\mathbf{X}^{n}, H^{n}}-t\left(\theta_{n}^{\mathbf{X}^{n}, H^{n}}\right)^{2}+\mathbf{i} t^{2} \kappa_{n}^{\mathbf{X}^{n}, H^{n}}\right)\left(\int_{0}^{t} e^{-\mathbf{i} x \mu_{n}^{\mathbf{X}^{n}, H^{n}}+\frac{x^{2}}{2}\left(\theta_{n}^{\mathbf{X}^{n}, H^{n}}\right)^{2}-\mathbf{i} \frac{x^{3}}{3} \kappa_{n}^{\mathbf{X}^{n}, H^{n}}} \bar{\varepsilon}_{n}^{\mathbf{X}^{n}, H^{n}}(x) d x\right)\right. \\
& \left.\times e^{\mathbf{i} t \mu_{n}^{\mathbf{X}^{n}, H^{n}}-\frac{t^{2}}{2}\left(\theta_{n}^{\mathbf{X}^{n}, H^{n}}\right)^{2}+\mathbf{i} \frac{t^{3}}{3} \kappa_{n}^{\mathbf{X}^{n}, H^{n}}}\right]+\mathbb{E}\left[\bar{\varepsilon}_{n}^{n}, H^{n}(t)\right] .
\end{aligned}
$$

Again, from Remark 7 in Appendix C,

$$
\begin{array}{r}
\operatorname{Var}\left[\mu_{n}^{\mathbf{X}^{n}, H^{n}}\right] \leq\left(\sqrt{\frac{n}{K} \operatorname{Var}\left[\log \operatorname{det}\left(\mathbf{I}_{N}+\frac{1}{\sigma^{2}} \frac{H H^{\mathrm{H}}}{K}\right)\right]}\right. \\
+\sqrt{\left.\frac{n}{K} \operatorname{Var}\left[\operatorname{tr} Q \frac{H \mathbf{A} H^{\mathrm{H}}}{K}\right]\right)^{2}} .
\end{array}
$$

From Proposition 3 (iii) in Appendix [E-A we know that $\operatorname{Var}\left[\operatorname{tr} Q \frac{H \mathbf{A} H^{\mathrm{H}}}{K}\right]=\mathcal{O}\left(\frac{1}{K} \operatorname{tr} \mathbf{A}^{2}\right)$. It remains to find a bound for the variance of the first term in (228). By Lemma 7 in Appendix [C]

$$
\begin{aligned}
& \operatorname{Var}\left[\log \operatorname{det}\left(\mathbf{I}_{N}+\frac{1}{\sigma^{2}} \frac{H H^{\mathrm{H}}}{K}\right)\right] \\
& \leq \frac{2}{\sigma^{4}} \sum_{i, j} \mathbb{E}\left[\left|\frac{1}{K} \operatorname{tr} Q \frac{\partial\left(H H^{\mathrm{H}}\right)}{\partial H_{i j}^{n}}\right|^{2}\right] \\
& =\frac{2}{\sigma^{4}} \sum_{i, j} \mathbb{E}\left[\left|\frac{1}{K} \sum_{p, q} \delta_{i p}\left(H_{q j}\right)^{*} Q_{q p}\right|^{2}\right] \\
& =\frac{2}{\sigma^{4}} \sum_{i, j} \mathbb{E}\left[\left|\frac{1}{K}\left[H^{\mathrm{H}} Q\right]_{j i}\right|^{2}\right] \\
& =\frac{2}{\sigma^{4}} \frac{1}{K} \operatorname{tr} Q^{2} \frac{H H^{\mathrm{H}}}{K} \\
& =\mathcal{O}(1) .
\end{aligned}
$$

Using the fact that $\operatorname{tr} \mathbf{A}^{2}=\mathcal{O}\left(n^{2}\right)$, we conclude that $\operatorname{Var}\left[\mu_{n}^{\mathbf{X}^{n}, H^{n}}\right]=\mathcal{O}(n)$.

Similarly, we have from Proposition 4 ( $i$ ) in Appendix E

$$
\begin{aligned}
\left|\mathbb{E}\left[\mu_{n}^{\mathbf{X}^{n}, H^{n}}\right]\right| & =\mid \sqrt{\frac{n}{K}} \mathbb{E}\left[\log \operatorname{det}\left(\mathbf{I}_{N}+\frac{1}{\sigma^{2}} \frac{H H^{\mathrm{H}}}{K}\right)\right. \\
& \left.-\sqrt{\frac{n}{K}} \operatorname{tr} Q \frac{H \mathbf{A} H^{\mathrm{H}}}{K}\right] \mid \\
& =\mathcal{O}\left(n+\sqrt{\frac{1}{n^{3}} \operatorname{tr} \mathbf{A}^{2}}\right) \\
& =\mathcal{O}(n) .
\end{aligned}
$$

Combining the last results, we have shown that

$$
\left|\mathbb{E}\left[\mu_{n}^{\mathbf{X}^{n}, H^{n}} \Theta\right]\right|=\mathcal{O}\left(t^{4} n^{-1}\right) .
$$

Similarly, one can show that

$$
\begin{aligned}
\left|\mathbb{E}\left[\left(\theta_{n}^{\mathbf{x}^{n}, H^{n}}\right)^{2} \Theta\right]\right| & =\mathcal{O}\left(t^{4} n^{-2}\right) \\
\left|\mathbb{E}\left[\kappa_{n}^{\mathbf{X}^{n}, H^{n}} \Theta\right]\right| & =\mathcal{O}\left(t^{4} n^{-3}\right) .
\end{aligned}
$$

Using (237), 238), and (239), we can finally conclude that

$$
\begin{aligned}
& \mathbb{E}\left[\left(\mathbf{i} \mu_{n}^{\mathbf{X}^{n}, H^{n}}-t\left(\theta_{n}^{\mathbf{X}^{n}, H^{n}}\right)^{2}+\mathbf{i} t^{2} \kappa_{n}^{\mathbf{X}^{n}, H^{n}}\right) \Theta\right] \\
& =\mathcal{O}\left(t^{4} n^{-1}+t^{5} n^{-2}+t^{6} n^{-3}\right) .
\end{aligned}
$$

Since all bounds are clearly integrable over $t$, this now means that $e^{\mathbf{i} t \mu_{n}^{\mathbf{X}^{n}, H^{n}}-\frac{t^{2}}{2}\left(\theta_{n}^{\mathbf{X}^{n}, H^{n}}\right)^{2}+\mathbf{i} \frac{t^{3}}{3} \kappa_{n}^{\mathbf{X}^{n}, H^{n}}}$ is an estimator of $\phi_{n}^{\mathbf{X}^{n}}$ within $\mathcal{O}\left(n^{-1}\right)$. Note that this bound would be $\mathcal{O}(1)$ if we had only used an estimation of $\phi_{n}^{\mathbf{X}^{n}, \mathbf{H}^{n}}$ within $\mathcal{O}\left(n^{-1}\right)$ in the previous step. Hence the fundamental importance of the term $\kappa_{n}^{\mathbf{X}^{n}, H^{n}}$.

We can therefore proceed to study $\phi_{n}^{\mathbf{X}^{n}}$ via the estimator $e^{\mathbf{i} t \mu_{n}^{\mathbf{X}^{n}}, H^{n}}-\frac{t^{2}}{2}\left(\theta_{n}^{\mathbf{X}^{n}, H^{n}}\right)^{2}+\mathbf{i} \frac{t^{3}}{3} \kappa_{n}^{\mathbf{X}^{n}, H^{n}}$.

Starting back from (222), we first verify that

$$
\begin{aligned}
& \mid \mathbb{E}\left[\mathbf{i} t^{2} \kappa_{n}^{\mathbf{X}^{n}, H^{n}} e^{\left.\mathbf{i} t \mu_{n}^{\mathbf{x}^{n}, H^{n}}-\frac{t^{2}}{2}\left(\theta_{n}^{\mathbf{X}^{n}, H^{n}}\right)^{2}+\mathbf{i} \frac{t^{3}}{3} \kappa_{n}^{\mathbf{X}^{n}, H^{n}}\right]}\right] \\
& =\mathcal{O}\left(t^{2} n^{-1}\right) \mathbb{E}\left[e^{-\frac{t^{2}}{2}\left(\theta_{n}^{\mathbf{X}^{n}, H^{n}}\right)^{2}}\right]=\mathcal{O}\left(t^{2} n^{-1}\right) .
\end{aligned}
$$

Thus, we have

$$
\begin{aligned}
\frac{\partial \phi_{n}^{\mathbf{X}^{n}}(t)}{\partial t}=\mathbb{E}[ & \left(\mathbf{i} \mu_{n}^{\mathbf{X}^{n}, H^{n}}-t\left(\theta_{n}^{\mathbf{X}^{n}, H^{n}}\right)^{2}\right) \\
& \left.\times e^{\mathbf{i} t \mu_{n}^{\mathbf{x}^{n}, H^{n}}-\frac{t^{2}}{2}\left(\theta_{n}^{\mathbf{X}^{n}, H^{n}}\right)^{2}+\mathbf{i} \frac{\mathbf{t}^{3}}{3} \kappa_{n}^{\mathbf{X}^{n}, H^{n}}}\right] \\
+ & \mathcal{O}\left(\frac{t^{2}+t^{4}}{n}+\frac{t^{3}+t^{5}}{n^{2}}+\frac{t^{6}}{n^{3}}\right) .
\end{aligned}
$$

We now develop the term in the expectation and express it under the form of $f(\mathbf{X}) \phi_{n}^{\mathbf{X}^{n}}(t)+\varepsilon_{n}^{\mathbf{X}^{n}}(t)$ for some functional $f$ and asymptotically negligible quantity $\varepsilon_{n}^{\mathbf{X}^{n}}(t)$. For better readability, we define the shorthand notation

$$
\gamma_{n}^{\mathbf{X}^{n}, H^{n}}=\mathbf{i} t \mu_{n}^{\mathbf{X}^{n}, H^{n}}-\frac{t^{2}}{2}\left(\theta_{n}^{\mathbf{X}^{n}, H^{n}}\right)^{2}+\mathbf{i} \frac{t^{3}}{3} \kappa_{n}^{\mathbf{X}^{n}, H^{n}}
$$

and consider individually the terms
A: $\mathbb{E}\left[\mu_{n}^{\mathbf{X}^{n}, H^{n}} e^{\gamma_{n}^{\mathbf{X}^{n}, H^{n}}}\right]$,
B: $\mathbb{E}\left[\left(\theta_{n}^{\mathbf{X}^{n}, H^{n}}\right)^{2} e^{\gamma_{n}^{\mathbf{X}^{n}, H^{n}}}\right]$. 
Term A: The term A cannot be evaluated in a straightforward manner as the integration by parts formula (Lemma 6 in Appendix C) cannot be applied to the log-term in $\mu_{n}^{\mathbf{X}^{n}, H^{n}}$ (as defined in (203)). To avert this difficulty, we use the identity

$$
\log \operatorname{det}\left(\mathbf{I}_{N}+\frac{1}{\sigma^{2}} \frac{H H^{\mathrm{H}}}{K}\right)=\int_{\sigma^{2}}^{\infty} \frac{1}{u} \operatorname{tr} Q(u) \frac{H H^{\mathrm{H}}}{K} d u
$$

which, together with the Fubini theorem (using $\left.\operatorname{tr} Q(u) H H^{\mathrm{H}} \leq u^{-1} \operatorname{tr} H H^{\mathrm{H}}\right)$, gives for $\mathrm{A}$ :

$$
\begin{aligned}
\mathbb{E} & {\left[\mu_{n}^{\mathbf{X}^{n}, H^{n}} e^{\left.\gamma_{n}^{\mathbf{x}^{n}, H^{n}}\right]}\right.} \\
= & \sqrt{\frac{n}{K}} \int_{\sigma^{2}}^{\infty} \frac{1}{u} \mathbb{E}\left[\operatorname{tr} Q(u) \frac{H H^{\mathrm{H}}}{K} e^{\gamma_{n}^{\mathbf{X}^{n}, H^{n}}}\right] d u \\
& -\sqrt{\frac{n}{K}} \mathbb{E}\left[\operatorname{tr} Q \frac{H \mathbf{A} H^{\mathrm{H}}}{K} e^{\gamma_{n}^{\mathbf{x}^{n}, H^{n}}}\right] .
\end{aligned}
$$

Before we continue, we need the following result which is the cornerstone of the subsequent analysis:

Proposition 2: Let $u \geq \sigma^{2}>0$ and $\gamma_{n}^{\mathbf{X}^{n}, H^{n}}$ be defined as in 243. Then,

$$
\begin{aligned}
& \mathbb{E}\left[\operatorname{tr} Q(u) \frac{H H^{\mathrm{H}}}{K} e^{\gamma_{n}^{\mathbf{x}^{n}, H^{n}}}\right] \\
& =N \frac{\left(1-c+2 u \delta_{0}(u)-\frac{u^{2}}{c} \delta_{0}(u)^{2}\right)}{1-c+u\left(1+2 \delta_{0}(u)\right)} \mathbb{E}\left[e^{\gamma_{n}^{\mathbf{x}^{n}, H^{n}}}\right] \\
& +\mathbf{i} t \sqrt{\frac{n}{K}} u \frac{\delta_{0}(u)-\sigma^{2} \delta_{1}(u)}{1-c+u\left(1+2 \delta_{0}(u)\right)} \mathbb{E}\left[e^{\gamma_{n}^{\mathbf{x}^{n}, H^{n}}}\right] \\
& +\mathcal{O}\left(\frac{P(t)}{u \sqrt{K}}\right) \\
& \text { (ii) } \mathbb{E}\left[\operatorname{tr} Q \frac{H \mathbf{A} H^{\mathrm{H}}}{K} e^{\gamma_{n}^{\mathbf{x}^{n}, H^{n}}}\right] \\
& =-\mathbf{i} t \sqrt{\frac{n}{K}} \frac{\gamma_{1}\left(\sigma^{2}\right) \frac{1}{K} \operatorname{tr} \mathbf{A}^{2}}{\left(1+\delta_{0}\left(\sigma^{2}\right)\right)^{2}} \mathbb{E}\left[e^{\gamma_{n}^{\mathbf{x}^{n}, H^{n}}}\right] \\
& +\mathcal{O}\left(\frac{P_{1}(t)}{\sqrt{K}}+\frac{t P_{2}(t)}{\sqrt{K}} \frac{1}{K} \operatorname{tr} \mathbf{A}^{2}\right)
\end{aligned}
$$

for some non-zero polynomials $P(t), P_{1}(t), P_{2}(t)$ in $t$ with nonnegative coefficients and with $\delta_{m}(x)$ and $\gamma_{m}(x)$ given by Proposition 4 in Appendix E

Proof: The proof is provided in Appendix E-B.

Applying Proposition $2(i)$ and $(i i)$ to the first and second terms of (245), respectively, we obtain the result in (248) on the top of the next page, where for the last RHS term, we used $\int_{\sigma^{2}}^{\infty} u^{-2} d u<\infty$ and $P_{1}, P_{2}$ are non-zero polynomials with nonnegative coefficients, possibly different from those of Proposition 2. Note in passing the fundamental importance of maintaining $1 / u$ in the big- $\mathcal{O}$ term of Proposition 2$](i)$. The existence of the two integrals in 248 can be proved via bounds on the $\delta_{t}(u)$ and $\gamma_{t}(u)$, essentially relying on their definitions in Proposition 4 and on controls similar to Property 1 (i) and (ii) in Appendix [C Nonetheless, a more immediate argument consists in remarking that, since the LHS of (248) is finite, and so are all terms aside from the integrals on the RHS, so is the sum of the integrals. Taking $t=0$ then justifies with the same argument that the first integral is finite which, taking then $t \neq 0$, ensures the finiteness of the second integral.
Also note that the last RHS term of (248) is not necessarily negligible in the large $n$ limit. Indeed, for $\mathbf{X} \in \mathcal{S}^{n}$, $\operatorname{tr} \mathbf{A}^{2}$ can grow as $\mathcal{O}\left(K^{2}\right)$, so that the whole term may grow as $\mathcal{O}(\sqrt{K})$. It is therefore essential to keep track of the terms in $\mathbf{A}$. The pre-factor $t$ in front of $\frac{1}{K} \operatorname{tr} \mathbf{A}^{2}$ will play a significant role in controlling these terms at the end of the proof, which explains why we also need to keep track of $t$ in the various bounds.

Term B: For the term B, we have from the identities in (152)

$$
\begin{aligned}
\mathbb{E} & {\left[\left(\theta_{n}^{\mathbf{X}^{n}, H^{n}}\right)^{2} e^{\gamma_{n}^{\mathbf{x}^{n}, H^{n}}}\right] } \\
= & \mathbb{E}\left[\left(\frac{1}{K} \operatorname{tr}\left(Q \frac{H H^{\mathrm{H}}}{K}\right)^{2}+\frac{2 \sigma^{2}}{K} \operatorname{tr}\left(Q \frac{H \mathbf{X} \mathbf{X}^{\mathrm{H}} H^{\mathrm{H}}}{n K} Q\right)\right)\right. \\
\left.\quad \times e^{\gamma_{n}^{\mathbf{x}^{n}, H^{n}}}\right] & \mathbb{E}\left[\left(\frac{1}{K} \operatorname{tr} Q \frac{H H^{\mathrm{H}}}{K}+\frac{\sigma^{2}}{K} \operatorname{tr} Q^{2} \frac{H H^{\mathrm{H}}}{K}\right.\right. \\
- & \left.\frac{2 \sigma^{2}}{K} \operatorname{tr} Q^{2} \frac{H\left(\mathbf{I}_{K}-\frac{1}{n} \mathbf{X} \mathbf{X}^{\mathrm{H}}\right) H^{\mathrm{H}}}{K}\right) e^{\left.\gamma_{n}^{\mathbf{x}^{n}, H^{n}}\right]+\mathcal{O}\left(\frac{1}{K}\right)} \\
= & \mathbb{E}\left[\left(c-\frac{\sigma^{4}}{K} \operatorname{tr} Q^{2}-\frac{2 \sigma^{2}}{K} \operatorname{tr} Q^{2} \frac{H \mathbf{A} H^{\mathrm{H}}}{K}\right) e^{\gamma_{n}^{\mathbf{x}^{n}, H^{n}}}\right] \\
& +\mathcal{O}\left(\frac{1}{K}\right) .
\end{aligned}
$$

To proceed with this term, which is essentially equal to the product of the expectations of the two arguments, we rely on Remark 7 in Appendix C. Using Proposition 3 in Appendix E-A and Proposition 4 in Appendix E-A to bound the variances of each term, we have

$$
\begin{aligned}
\mathbb{E}\left[\left(\theta_{n}^{\mathbf{X}^{n}, H^{n}}\right)^{2} e^{\gamma_{n}^{\mathbf{x}^{n}, H^{n}}}\right]= & \left(c-\sigma^{4} \delta_{1}\left(\sigma^{2}\right)\right) \mathbb{E}\left[e^{\gamma_{n}^{\mathbf{x}^{n}, H^{n}}}\right] \\
& +\mathcal{O}\left(\frac{1}{\sqrt{K}}\right)
\end{aligned}
$$

where we used in particular $\sqrt{K^{-3} \operatorname{tr} \mathbf{A}^{2}} \leq 1 / \sqrt{K}$.

Combining (242), 248, and (252) we finally obtain the differential equation 253) on the next page, where $\mu_{n}^{\mathbf{X}^{n}}$, $\left(\theta_{n}^{\mathbf{X}^{n}}\right)^{2}$, and $\bar{\varepsilon}_{n}^{\mathbf{X}^{n}}(t)$ are defined in 254-256) for some non-zero polynomials with nonnegative coefficients $P_{1}(t)$ and $P_{2}(t)$.

Using Lemma 9 in Appendix $\mathbb{C}$ and the definition of $\gamma_{1}, \delta_{1}$ in Proposition 4 the expressions of $\mu_{n}^{\mathbf{X}^{n}}$ and $\theta_{n}^{\mathbf{X}^{n}}$ can be simplified as:

$$
\begin{aligned}
\mu_{n}^{\mathbf{X}^{n}} & =\sqrt{n K} C \\
\theta_{n}^{\mathbf{X}^{n}} & =\left[\theta_{-}^{2}+\zeta \frac{1}{K} \operatorname{tr} \mathbf{A}^{2}\right]^{\frac{1}{2}}
\end{aligned}
$$

where $\theta_{-}$is defined in the statement of Theorem 3 and $\zeta=$ $-\delta_{0}^{\prime}\left(\sigma^{2}\right)\left(1+\delta_{0}\left(\sigma^{2}\right)\right)^{-1}$. Note that we have used the relation $\delta_{0}\left(\sigma^{2}\right)=-\delta_{1}\left(\sigma^{2}\right)$. Before we continue with the main proof, we will show that $\theta_{-}^{2}>0$ and $\zeta>0$.

For the former, first note that the logarithm term of $\theta_{-}$ in (24) is well defined. Indeed, for $c \geq 1$, the argument is 


$$
\begin{aligned}
\mathbb{E}\left[\mu_{n}^{\mathbf{X}^{n}, H^{n}} e^{\left.\gamma_{n}^{\mathbf{x}^{n}, H^{n}}\right]=}\right. & \sqrt{n K}\left\{\int_{\sigma^{2}}^{\infty} \frac{c\left(1-c+2 u \delta_{0}(u)-\frac{u^{2}}{c} \delta_{0}(u)^{2}\right)}{u\left(1-c+u\left(1+2 \delta_{0}(u)\right)\right)} d u\right\} \mathbb{E}\left[e^{\gamma_{n}^{\mathbf{x}^{n}, H^{n}}}\right] \\
& +\mathbf{i} t \frac{n}{K}\left\{\int_{\sigma^{2}}^{\infty} \frac{\delta_{0}(u)-\sigma^{2} \delta_{1}(u)}{1-c+u\left(1+2 \delta_{0}(u)\right)} d u+\frac{\gamma_{1}\left(\sigma^{2}\right) \frac{1}{K} \operatorname{tr} \mathbf{A}^{2}}{\left(1+\delta_{0}\left(\sigma^{2}\right)\right)^{2}}\right\} \mathbb{E}\left[e^{\left.\gamma_{n}^{\mathbf{x}^{n}, H^{n}}\right]}\right. \\
& +\mathcal{O}\left(\frac{P_{1}(t)}{\sqrt{K}}+\frac{t P_{2}(t)}{\sqrt{K}} \frac{1}{K} \operatorname{tr} \mathbf{A}^{2}\right)
\end{aligned}
$$

$$
\begin{aligned}
\frac{\partial \phi_{n}^{\mathbf{X}^{n}}(t)}{\partial t} & =\left(\mathbf{i} \mu_{n}^{\mathbf{X}^{n}}-t\left(\theta_{n}^{\mathbf{X}^{n}}\right)^{2}\right) \mathbb{E}\left[e^{\gamma_{n}^{\mathbf{X}^{n}, H^{n}}}\right]+\bar{\varepsilon}_{n}^{\mathbf{X}^{n}}(t) \\
\mu_{n}^{\mathbf{X}^{n}} & =\sqrt{n K}\left\{\int_{\sigma^{2}}^{\infty} \frac{c\left(1-c+2 u \delta_{0}(u)-\frac{u^{2}}{c} \delta_{0}(u)^{2}\right)}{u\left(1-c+u\left(1+2 \delta_{0}(u)\right)\right)} d u\right\} \\
\left(\theta_{n}^{\mathbf{X}^{n}}\right)^{2} & =\frac{n}{K}\left\{\int_{\sigma^{2}}^{\infty} \frac{\delta_{0}(u)-\sigma^{2} \delta_{1}(u)}{1-c+u\left(1+2 \delta_{0}(u)\right)} d u+\frac{\gamma_{1}\left(\sigma^{2}\right) \frac{1}{K} \operatorname{tr} \mathbf{A}^{2}}{\left(1+\delta_{0}\left(\sigma^{2}\right)\right)^{2}}+\frac{K}{n}\left(c-\sigma^{4} \delta_{1}\left(\sigma^{2}\right)\right)\right\} \\
\bar{\varepsilon}_{n}^{\mathbf{X}^{n}}(t) & =\mathcal{O}\left(\frac{P_{1}(t)}{\sqrt{K}}+\frac{t P_{2}(t)}{\sqrt{K}} \frac{1}{K} \operatorname{tr} \mathbf{A}^{2}\right)
\end{aligned}
$$

clearly positive. For $c<1$, by Property $1(i v)$ in Appendix C $\delta_{0}\left(\sigma^{2}\right)^{2}\left(1+\delta_{0}\left(\sigma^{2}\right)\right)^{-2}=\left(c-\sigma^{2} \delta_{0}\left(\sigma^{2}\right)\right)^{2}<c^{2}$, with the inequality arising from Property $1(i)$ and $(i i)$ in Appendix C: this then implies that the argument is greater than $1-c>0$. Obviously, in both cases, as the argument of the logarithm is less than one, the logarithm itself is negative. This implies that

$$
\begin{aligned}
\theta_{-}^{2} & =-\beta \log \left(1-\frac{1}{c} \frac{\delta_{0}\left(\sigma^{2}\right)^{2}}{\left(1+\delta_{0}\left(\sigma^{2}\right)\right)^{2}}\right)+\left(c+\sigma^{4} \delta_{0}^{\prime}\left(\sigma^{2}\right)\right) \\
& \stackrel{(a)}{>} c-\frac{\sigma^{4} \delta_{0}\left(\sigma^{2}\right)\left(1+\delta_{0}\left(\sigma^{2}\right)\right)}{1-c+\sigma^{2}\left(1+\delta_{0}\left(\sigma^{2}\right)\right)+\sigma^{2} \delta_{0}\left(\sigma^{2}\right)} \\
& \stackrel{(b)}{=} c-\frac{c \sigma^{2}\left(1+\delta_{0}\left(\sigma^{2}\right)\right)}{\frac{c}{\delta_{0}\left(\sigma^{2}\right)}+\sigma^{2} \delta_{0}\left(\sigma^{2}\right)} \\
& \stackrel{(c)}{>} c\left(1-\frac{\sigma^{2}\left(1+\delta_{0}\left(\sigma^{2}\right)\right)}{\sigma^{2}+\sigma^{2} \delta_{0}\left(\sigma^{2}\right)}\right) \\
& =0
\end{aligned}
$$

where $(a)$ follows from the definition of $\delta_{0}^{\prime}(x)$ established in Property 1 (vi) in Appendix [C, (b) follows from Property 1 (iii) in Appendix [C] and (c) is due to Property 1 (ii) in Appendix $\mathbb{C}$ which implies that $\frac{c}{\delta_{0}\left(\sigma^{2}\right)}>\sigma^{2}$.

Concerning $\zeta$, we first show that $\delta_{1}\left(\sigma^{2}\right)=-\delta_{0}^{\prime}\left(\sigma^{2}\right)>0$ (where this identity follows from Property $1(v i)$ in Appendix [C). Since $c^{-1} \delta_{0}\left(\sigma^{2}\right)$ is the Stieltjes transform of the Marčenko-Pastur law $\mu_{c}$ taken in $-\sigma^{2}$ (see, e.g., [28, Chapter $3.2]$ ), we can conclude that

$$
\delta_{1}\left(\sigma^{2}\right)=-\delta_{0}^{\prime}\left(\sigma^{2}\right)=\frac{1}{c} \int \frac{1}{\left(t+\sigma^{2}\right)^{2}} \mu_{c}(d t)>0 .
$$

Since also $\delta_{0}\left(\sigma^{2}\right)>0$, it follows that $\zeta=-\beta \delta_{0}^{\prime}\left(\sigma^{2}\right)(1+$ $\left.\delta_{0}\left(\sigma^{2}\right)\right)>0$.
We now relate $\phi_{n}^{\mathbf{X}^{n}}(t)=\mathbb{E}\left[\phi_{n}^{\mathbf{X}^{n}, H^{n}}(t)\right]$ and $\mathbb{E}\left[e^{\gamma_{n}^{\mathbf{X}^{n}, H^{n}}}\right]$ with the help of the previously established results. Starting from (220), one can easily show that

$$
\left|\phi_{n}^{\mathbf{X}^{n}, \mathbf{H}^{n}}(t)-e^{\gamma_{n}^{\mathbf{x}^{n}, \mathbf{H}^{n}}}\right| \leq M t^{4} n^{-2}
$$

for some constant $M$ independent of $\mathbf{H}, t$, and $n$, from which

$$
\phi_{n}^{\mathbf{X}^{n}}(t)=\mathbb{E}\left[\phi_{n}^{\mathbf{X}^{n}, H^{n}}(t)\right]=\mathbb{E}\left[e^{\gamma_{n}^{\mathbf{X}^{n}, H^{n}}}\right]+\mathcal{O}\left(t^{4} n^{-2}\right)
$$

or, equivalently,

$$
\mathbb{E}\left[e^{\gamma_{n}^{\mathbf{X}^{n}, H^{n}}}\right]=\phi_{n}^{\mathbf{X}^{n}}(t)+\mathcal{O}\left(t^{4} n^{-2}\right) .
$$

Replacing the last equation in (253) leads to

$$
\begin{aligned}
\frac{\partial \phi_{n}^{\mathbf{X}^{n}}(t)}{\partial t}= & \left(\mathbf{i} \mu_{n}^{\mathbf{X}^{n}}-t\left(\theta_{n}^{\mathbf{X}^{n}}\right)^{2}\right) \phi_{n}^{\mathbf{X}^{n}}(t) \\
& +\left(\mathbf{i} \mu_{n}^{\mathbf{X}^{n}}-t\left(\theta_{n}^{\mathbf{X}^{n}}\right)^{2}\right) \mathcal{O}\left(t^{4} n^{-2}\right)+\bar{\varepsilon}_{n}^{\mathbf{X}^{n}}(t) .
\end{aligned}
$$

One can verify from (254) and (255) that

$$
\begin{aligned}
\mu_{n}^{\mathbf{X}^{n}} & =\mathcal{O}(n) \\
\left(\theta_{n}^{\mathbf{X}^{n}}\right)^{2} & =\mathcal{O}\left(1+\frac{1}{K} \operatorname{tr} \mathbf{A}^{2}\right) .
\end{aligned}
$$

Hence

$$
\frac{\partial \phi_{n}^{\mathbf{X}^{n}}(t)}{\partial t}=\left(\mathbf{i} \mu_{n}^{\mathbf{X}^{n}}-t\left(\theta_{n}^{\mathbf{X}^{n}}\right)^{2}\right) \phi_{n}^{\mathbf{X}^{n}}(t)+\varepsilon_{n}^{\mathbf{X}^{n}}(t)
$$

where $\varepsilon_{n}^{\mathbf{X}^{n}}(t)$ satisfies

$$
\varepsilon_{n}^{\mathbf{X}^{n}}(t)=\mathcal{O}\left(\frac{P_{1}(t)}{\sqrt{K}}+\frac{t P_{2}(t)}{\sqrt{K}} \frac{1}{K} \operatorname{tr} \mathbf{A}^{2}\right) .
$$


Step 3:

Solving the differential equation 271, we arrive at

$$
\begin{aligned}
\phi_{n}^{\mathbf{X}^{n}}(t)= & e^{\mathbf{i} t \mu_{n}^{\mathbf{X}^{n}}-\frac{t^{2}}{2}\left(\theta_{n}^{\mathbf{X}^{n}}\right)^{2}}(1+ \\
& \left.\int_{0}^{t} e^{-\mathbf{i} x \mu_{n}^{\mathbf{X}^{n}}+\frac{x^{2}}{2}\left(\theta_{n}^{\mathbf{X}^{n}}\right)^{2}} \varepsilon_{n}^{\mathbf{X}^{n}}(x) d x\right) \\
= & e^{\mathbf{i} t \mu_{n}^{\mathbf{X}^{n}}-\frac{t^{2}}{2}\left(\theta_{n}^{\mathbf{X}^{n}}\right)^{2}}+\tilde{\varepsilon}_{n}^{\mathbf{X}^{n}}(t)
\end{aligned}
$$

with $\tilde{\varepsilon}_{n}^{\mathbf{X}^{n}}(t)=\mathcal{O}\left(t \varepsilon_{n}^{\mathbf{X}^{n}}(t)\right)$.

Denote $\tilde{\phi}_{n}^{\mathbf{X}^{n}}(t)=\mathbb{E}\left[e^{\mathbf{i} \frac{t}{\theta_{n}^{\mathbf{X}^{n}}}\left(\Gamma_{n}^{\mathbf{X}^{n}}-\mu_{n}^{\mathbf{X}^{n}}\right)}\right]$. Then, from (274),

$$
\begin{aligned}
\tilde{\phi}_{n}^{\mathbf{X}^{n}}(t) & =\mathbb{E}\left[e^{\mathbf{i} \frac{t}{\theta_{n}^{\mathbf{X}^{n}}}\left(\Gamma_{n}^{\mathbf{X}^{n}}-\mu_{n}^{\mathbf{X}^{n}}\right)}\right] \\
& =\phi_{n}^{\mathbf{X}^{n}}\left(\frac{t}{\theta_{n}^{\mathbf{X}^{n}}}\right) e^{-\mathbf{i} t \frac{\mu_{n}^{\mathbf{X}^{n}}}{\theta_{n}^{\mathbf{X}^{n}}}} \\
& =e^{-\frac{t^{2}}{2}}+\tilde{\varepsilon}_{n}^{\mathbf{X}^{n}}\left(\frac{t}{\theta_{n}^{\mathbf{X}^{n}}}\right) e^{-\mathbf{i} t \frac{\mu_{n}^{\mathbf{X}^{n}}}{\theta_{n}^{\mathbf{X}^{n}}}} .
\end{aligned}
$$

To conclude, we need to control the term $\tilde{\varepsilon}_{n}^{\mathbf{X}^{n}}\left(t\left(\theta_{n}^{\mathbf{X}^{n}}\right)^{-1}\right)$. This is where the precision on $\varepsilon_{n}^{\mathbf{X}_{n}}(t)$ from (272) is used. Take $t \geq 0$ fixed. First, observe from (263) that $\theta_{n}^{\mathbf{X}^{n}} \geq \theta_{-}>0$.

We then have

$$
\begin{aligned}
& \tilde{\varepsilon}_{n}^{\mathbf{X}^{n}}\left(\frac{t}{\theta_{n}^{\mathbf{X}^{n}}}\right) \\
& =\mathcal{O}\left(\frac{t}{\theta_{n}^{\mathbf{X}^{n}}} \varepsilon_{n}^{\mathbf{X}^{n}}\left(\frac{t}{\theta_{n}^{\mathbf{X}^{n}}}\right)\right) \\
& =\mathcal{O}\left(\frac{P_{1}\left(t\left(\theta_{n}^{\mathbf{X}^{n}}\right)^{-1}\right)}{\sqrt{K} \theta_{n}^{\mathbf{X}^{n}}}+\frac{P_{2}\left(t\left(\theta_{n}^{\mathbf{X}^{n}}\right)^{-1}\right)}{\sqrt{K}\left(\theta_{n}^{\mathbf{X}^{n}}\right)^{2}} \frac{1}{K} \operatorname{tr} \mathbf{A}^{2}\right) \\
& =\mathcal{O}\left(\frac{1}{\sqrt{K}}\right)
\end{aligned}
$$

where, in the last equality, we used $P_{1}\left(t\left(\theta_{n}^{\mathbf{X}^{n}}\right)^{-1}\right)\left(\theta_{n}^{\mathbf{X}^{n}}\right)^{-1} \leq$ $P_{1}\left(t \theta_{-}^{-1}\right) \theta_{-}^{-1}, P_{2}\left(t\left(\theta_{n}^{\mathbf{X}^{n}}\right)^{-1}\right) \leq P_{2}\left(t \theta_{-}^{-1}\right)$, both bounded for $t$ fixed, and

$$
\frac{\frac{1}{K} \operatorname{tr} \mathbf{A}^{2}}{\left(\theta_{n}^{\mathbf{X}^{n}}\right)^{2}}=\frac{\frac{1}{K} \operatorname{tr} \mathbf{A}^{2}}{\theta_{-}^{2}+\zeta \frac{1}{K} \operatorname{tr} \mathbf{A}^{2}} \leq \frac{1}{\zeta}<\infty .
$$

We conclude that

$$
\left|\tilde{\varepsilon}_{n}^{\mathbf{X}^{n}}\left(\frac{t}{\theta_{n}^{\mathbf{X}^{n}}}\right) e^{-\mathbf{i} t \frac{\mu_{n}^{\mathbf{X}^{n}}}{\theta_{n}^{\mathbf{X}^{n}}}}\right|=\mathcal{O}\left(\frac{1}{\sqrt{K}}\right) .
$$

Take now $\mathbb{P}_{X^{n}} \in \mathcal{P}\left(\mathcal{S}_{=}^{n}\right)$ for all $n$ and let $\tilde{\phi}_{n}(t) \triangleq \mathbb{E}\left[\tilde{\phi}_{n}^{X^{n}}(t)\right]$. Then, from (277) and (282),

$$
\tilde{\phi}_{n}(t)=e^{-\frac{t^{2}}{2}}+\mathcal{O}\left(\frac{1}{\sqrt{K}}\right) .
$$

Taking $t<0$, and using $\tilde{\phi}_{n}(-t)=\tilde{\phi}_{n}(t)^{*}$, the result above generalizes to $t \in \mathbb{R}$.

This implies by Lévy's continuity theorem that

$$
\frac{\Gamma_{n}-\mu_{n}}{\theta_{n}} \Rightarrow \mathcal{N}(0,1)
$$

where we have defined $\mu_{n}=\mu_{n}^{X^{n}}$ and $\theta_{n}=\theta_{n}^{X^{n}}$. This terminates the proof.

\section{APPENDIX E}

ADDITIONAL RANDOM MATRIX RESULTS

\section{A. Auxiliary results}

Lemma 10: Let $G \in \mathbb{C}^{M \times L}$ have i.i.d. entries $G_{i j} \sim$ $\mathcal{C N}(0,1)$ and let $\mathbf{S} \in \mathbb{C}^{L \times M}$ and $\mathbf{T} \in \mathbb{C}^{M \times M}$. Then,

$$
\begin{aligned}
\text { (i) } \quad \operatorname{Var}[\operatorname{tr} \mathbf{S} G] & =\operatorname{tr} \mathbf{S S}^{\mathbf{H}} \\
\text { (ii) } \quad \operatorname{Var}\left[\operatorname{tr} \mathbf{T} G G^{\mathrm{H}}\right] & \leq 2 L \operatorname{tr} \mathbf{T} \mathbf{T}^{\mathrm{H}} .
\end{aligned}
$$

Proof: The proof of part $(i)$ is obvious. Part $(i i)$ is proved by a mere application of Lemma 7 and Lemma 8 in Appendix C

Lemma 11: Let $G \in \mathbb{C}^{M \times L}$ have i.i.d. entries $G_{i j} \sim$ $\mathcal{C N}(0,1)$. Let $\mathbf{T} \in \mathbb{C}^{M \times M}$ be a deterministic matrix and $\omega$ be a function of $G$. Then,

$$
\begin{aligned}
\mathbb{E}\left[\operatorname{tr} \mathbf{T} G G^{\mathrm{H}} e^{\omega}\right]= & L \operatorname{tr} \mathbf{T} \mathbb{E}\left[e^{\omega}\right] \\
& +\mathbb{E}\left[\sum_{i, j} \frac{\partial \omega}{\partial G_{i j}^{*}}\left[G^{\mathrm{H}} \mathbf{T}\right]_{j i} e^{\omega}\right] .
\end{aligned}
$$

Proof: This follows immediately from Lemma 6 in Appendix C

Proposition 3: Let $H \in \mathbb{C}^{N \times K}$ have i.i.d. elements $H_{i j} \sim \mathcal{C N}(0,1)$ and define the functionals $Q(x)=$ $\left(\frac{1}{K} H H^{\mathrm{H}}+x \mathbf{I}_{N}\right)^{-1}$ and $\tilde{Q}(x)=\left(\frac{1}{K} H^{\mathrm{H}} H+x \mathbf{I}_{K}\right)^{-1}$ for $x>0$. Further, let $\mathbf{C}, \mathbf{D} \in \mathbb{C}^{N \times N}$ and $\tilde{\mathbf{C}}, \tilde{\mathbf{D}} \in \mathbb{C}^{K \times K}$. Then, for $u, v>0$ and any nonnegative integer $m$, the following holds:

$$
\begin{aligned}
\text { (i) } \operatorname{Var}[ & \left.\frac{1}{K} \operatorname{tr} \mathbf{C} Q(u) \mathbf{D} Q(v)^{m}\right] \\
& \leq 2 \frac{\left(\sqrt{\frac{v}{u}}+m\right)^{2}}{u^{2} v^{2 m+1}} \frac{\|\mathbf{D}\|^{2}}{K^{3}} \operatorname{tr} \mathbf{C C}^{\mathrm{H}} \\
\text { (ii) } \operatorname{Var}[ & \left.\frac{1}{K} \operatorname{tr} \tilde{\mathbf{C}} \tilde{Q}(u) \tilde{\mathbf{D}} \tilde{Q}(v)^{m}\right] \\
& \leq 2 \frac{\left(\sqrt{\frac{v}{u}}+m\right)^{2}}{u^{2} v^{2 m+1}} \frac{\|\tilde{\mathbf{D}}\|^{2}}{K^{3}} \operatorname{tr} \tilde{\mathbf{C}} \tilde{\mathbf{C}}^{\mathrm{H}} \\
\text { (iii) } \operatorname{Var} & {\left[\frac{1}{K} \operatorname{tr} Q(u) Q(v)^{m} \frac{H \tilde{\mathbf{C}} H^{\mathrm{H}}}{K}\right] } \\
& \leq 2 \frac{\left(\sqrt{\frac{v}{u}}+2 m\right)^{2}}{v^{2 m+1}} \frac{1}{K^{3}} \operatorname{tr} \tilde{\mathbf{C}} \tilde{\mathbf{C}}^{\mathrm{H}} \\
& {\left[\frac{1}{K} \operatorname{tr} Q(u) Q(v)^{m} \frac{H \tilde{\mathbf{C}} H^{\mathrm{H}}}{K}\right] } \\
\text { (iv) } \operatorname{Var} & \leq 2 \frac{\left(2 \sqrt{\frac{v}{u}}+2 m-1\right)^{2}}{u^{2} v^{2 m-1}} \frac{1}{K^{3}} \operatorname{tr} \tilde{\mathbf{C}} \tilde{\mathbf{C}}^{\mathrm{H}}, \quad m \geq 1 .
\end{aligned}
$$

Moreover, for $\mathbf{C}$ and $\tilde{\mathbf{C}}$ Hermitian,

(v) $\operatorname{Var}\left[\frac{1}{K} \operatorname{tr} \mathbf{C} Q(u) \mathbf{C} Q(v)^{m}\right] \leq 2 \frac{\left(\sqrt{\frac{v}{u}}+m\right)^{2}}{u^{2} v^{2 m+1}} \frac{1}{K^{3}} \operatorname{tr} \mathbf{C}^{4}$

(vi) $\operatorname{Var}\left[\frac{1}{K} \operatorname{tr} \tilde{\mathbf{C}} \tilde{Q}(u) \tilde{\mathbf{C}} \tilde{Q}(v)^{m}\right] \leq 2 \frac{\left(\sqrt{\frac{v}{u}}+m\right)^{2}}{u^{2} v^{2 m+1}} \frac{1}{K^{3}} \operatorname{tr} \tilde{\mathbf{C}}^{4}$. 
Proof: The results follow from the successive applications of Lemma 7 and Lemma 4 in Appendix C.

Proposition 4: Let $\left\{H^{n}\right\}_{n=1}^{\infty}$, where $H^{n} \in \mathbb{C}^{N \times K}$ has i.i.d. elements $H_{i j}^{n} \sim \mathcal{C N}(0,1)$, and define $Q^{n}(x)=$ $\left(\frac{1}{K} H^{n}\left(H^{n}\right)^{\mathrm{H}}+x \mathbf{I}_{N}\right)^{-1}$ for $x>0$. Let $\left\{\mathbf{C}^{n}\right\}_{n=1}^{\infty}$, where $\mathbf{C}^{n} \in \mathbb{C}^{N \times N}$. Then, for $u \geq \sigma^{2}>0$ and any nonnegative integer $m$, the following holds as $n \stackrel{(\beta, c)}{\longrightarrow} \infty$ :

$$
\text { (i) } \begin{aligned}
& \mathbb{E}\left[\frac{1}{K} \operatorname{tr} Q^{n}(u) Q^{n}\left(\sigma^{2}\right)^{m} \frac{H^{n} \mathbf{C}^{n}\left(H^{n}\right)^{\mathrm{H}}}{K}\right] \\
= & \gamma_{m}(u) \frac{1}{K} \operatorname{tr} \mathbf{C}^{n}+\mathcal{O}\left(\sqrt{\frac{1}{u^{2} K^{5}} \operatorname{tr}^{n} \mathbf{C}^{n}\left(\mathbf{C}^{n}\right)^{\mathrm{H}}}\right)
\end{aligned}
$$

$$
\text { (ii) } \mathbb{E}\left[\frac{1}{K} \operatorname{tr} Q^{n}(u) Q^{n}\left(\sigma^{2}\right)^{m}\right]=\delta_{m}(u)+\mathcal{O}\left(\frac{1}{u n^{2}}\right)
$$

where, for $m \geq 1$,

$$
\begin{aligned}
\gamma_{m}(u)= & \delta_{m-1}(u)-\sigma^{2} \delta_{m}(u) \\
\delta_{m}(u)= & \frac{\delta_{m-1}(u)\left[1+\delta_{0}\left(\sigma^{2}\right)\right]}{1-c+\sigma^{2}\left[1+\delta_{0}\left(\sigma^{2}\right)\right]+u \delta_{0}(u)} \\
& +\frac{\sum_{k=1}^{m-1}\left[\delta_{k-1}(u)-\sigma^{2} \delta_{k}(u)\right] \delta_{m-k}\left(\sigma^{2}\right)}{1-c+\sigma^{2}\left[1+\delta_{0}\left(\sigma^{2}\right)\right]+u \delta_{0}(u)}
\end{aligned}
$$

and

$$
\gamma_{0}(u)=c-u \delta_{0}(u)
$$

with $\delta_{0}(u)$ as defined in Theorem 4 in Appendix C.

Proof: In order to simplify the notations, we drop the dependence of $n$, e.g., we write $H$ instead of $H^{n}$. We begin by standard Gaussian calculus based on the integration by parts formula (Lemma 6 in Appendix C):

$$
\begin{aligned}
\mathbb{E} & {\left[\frac{1}{K} \operatorname{tr} Q(u) Q\left(\sigma^{2}\right)^{m} \frac{H \mathbf{C} H^{\mathrm{H}}}{K}\right] } \\
= & \frac{1}{K^{2}} \sum_{i, j, k, r, s} \mathbb{E}\left[H_{i j} C_{j k} H_{r k}^{*} Q(u)_{r s}\left[Q\left(\sigma^{2}\right)^{m}\right]_{s i}\right] \\
= & \frac{1}{K^{2}} \sum_{i, j, k, r, s} C_{j k} \mathbb{E}\left[\frac{\partial\left(H_{r k}^{*} Q(u)_{r s}\left[Q\left(\sigma^{2}\right)^{m}\right]_{s i}\right)}{\partial H_{i j}^{*}}\right] \\
= & \frac{1}{K^{2}} \sum_{i, j, k, r, s} C_{j k} \mathbb{E}\left[\delta_{i r} \delta_{j k} Q(u)_{l s}\left[Q\left(\sigma^{2}\right)^{m}\right]_{s i}\right. \\
& -\frac{1}{K} H_{r k}^{*}[Q(u) H]_{r j} Q(u)_{i s}\left[Q\left(\sigma^{2}\right)^{m}\right]_{s i} \\
& \left.+H_{r k}^{*} Q(u)_{r s} \frac{\partial\left[Q\left(\sigma^{2}\right)^{m}\right]_{s i}}{\partial H_{i j}^{*}}\right] \\
= & \frac{1}{K} \operatorname{tr} \mathbf{C} \mathbb{E}\left[\frac{1}{K} \operatorname{tr} Q(u) Q\left(\sigma^{2}\right)^{m}\right] \\
& -\mathbb{E}\left[\frac{1}{K} \operatorname{tr} Q(u) \frac{H \mathbf{C} H^{\mathrm{H}}}{K} \frac{1}{K} \operatorname{tr} Q(u) Q\left(\sigma^{2}\right)^{m}\right] \\
& +\frac{1}{K^{2}} \sum_{i, j, s} \mathbb{E}\left[\left[\mathbf{C} H^{\mathrm{H}} Q(u)\right]_{j s} \frac{\partial\left[Q\left(\sigma^{2}\right)^{m}\right]_{s i}}{\partial H_{i j}^{*}}\right] .
\end{aligned}
$$

To continue, we will develop the term $\frac{\partial\left[Q\left(\sigma^{2}\right)^{m}\right]_{s i}}{\partial H_{i j}^{*}}$ as follows:

$$
\begin{aligned}
& \frac{\partial\left[Q\left(\sigma^{2}\right)^{m}\right]_{s i}}{\partial H_{i j}^{*}} \\
& =\sum_{k=1}^{m} \sum_{p, q}\left[Q\left(\sigma^{2}\right)^{k-1}\right]_{s p} \frac{\partial Q\left(\sigma^{2}\right)_{p q}}{\partial H_{i j}^{*}}\left[Q\left(\sigma^{2}\right)^{m-k}\right]_{q i} \\
& =-\frac{1}{K} \sum_{k=1}^{m} \sum_{p, q}\left[Q\left(\sigma^{2}\right)^{k-1}\right]_{s p}\left[Q\left(\sigma^{2}\right) H\right]_{p j} \\
& \times Q\left(\sigma^{2}\right)_{i q}\left[Q\left(\sigma^{2}\right)^{m-k}\right]_{q i} \\
& =-\frac{1}{K} \sum_{k=1}^{m}\left[Q\left(\sigma^{2}\right)^{k} H\right]_{s j}\left[Q\left(\sigma^{2}\right)^{m-k+1}\right]_{i i} .
\end{aligned}
$$

Replacing (305) in 302, we arrive at

$$
\begin{aligned}
\mathbb{E} & {\left[\frac{1}{K} \operatorname{tr} Q(u) Q\left(\sigma^{2}\right)^{m} \frac{H \mathbf{C} H^{\mathrm{H}}}{K}\right] } \\
= & \frac{1}{K} \operatorname{tr} \mathbf{C} \mathbb{E}\left[\frac{1}{K} \operatorname{tr} Q(u) Q\left(\sigma^{2}\right)^{m}\right] \\
& -\mathbb{E}\left[\frac{1}{K} \operatorname{tr} Q(u) \frac{H \mathbf{C} H^{\mathrm{H}}}{K} \frac{1}{K} \operatorname{tr} Q(u) Q\left(\sigma^{2}\right)^{m}\right] \\
& -\sum_{k=1}^{m} \mathbb{E}\left[\frac{1}{K} \operatorname{tr} Q(u) Q\left(\sigma^{2}\right)^{k} \frac{H \mathbf{C} H^{\mathrm{H}}}{K} \frac{1}{K} \operatorname{tr} Q\left(\sigma^{2}\right)^{m-k+1}\right] .
\end{aligned}
$$

By Proposition 3 in Appendix E-A and Remark 7 in Appendix [C, we have

$$
\begin{aligned}
\mathbb{E} & {\left[\frac{1}{K} \operatorname{tr} Q(u) \frac{H \mathbf{C} H^{\mathrm{H}}}{K} \frac{1}{K} \operatorname{tr} Q(u) Q\left(\sigma^{2}\right)^{m}\right] } \\
=\mathbb{E} & {\left[\frac{1}{K} \operatorname{tr} Q(u) \frac{H \mathbf{C} H^{\mathrm{H}}}{K}\right] \mathbb{E}\left[\frac{1}{K} \operatorname{tr} Q(u) Q\left(\sigma^{2}\right)^{m}\right] } \\
& +\mathcal{O}\left(\sqrt{\frac{1}{u^{3} K^{5}} \operatorname{tr} \mathbf{C C}^{\mathrm{H}}}\right) \\
\mathbb{E} & {\left[\frac{1}{K} \operatorname{tr} Q(u) Q^{k} \frac{H \mathbf{C} H^{\mathrm{H}}}{K} \frac{1}{K} \operatorname{tr} Q^{m-k+1}\right] } \\
= & \mathbb{E}\left[\frac{1}{K} \operatorname{tr} Q(u) Q^{k} \frac{H \mathbf{C} H^{\mathrm{H}}}{K}\right] \mathbb{E}\left[\frac{1}{K} \operatorname{tr} Q^{m-k+1}\right] \\
& +\mathcal{O}\left(\sqrt{\frac{1}{u^{2} K^{5}} \operatorname{tr} \mathbf{C C}^{\mathrm{H}}}\right)
\end{aligned}
$$

and, thus,

$$
\begin{aligned}
\mathbb{E} & {\left[\frac{1}{K} \operatorname{tr} Q(u) Q\left(\sigma^{2}\right)^{m} \frac{H \mathbf{C} H^{\mathrm{H}}}{K}\right] } \\
= & \frac{1}{K} \operatorname{tr} \mathbf{C} \mathbb{E}\left[\frac{1}{K} \operatorname{tr} Q(u) Q\left(\sigma^{2}\right)^{m}\right] \\
& -\mathbb{E}\left[\frac{1}{K} \operatorname{tr} Q(u) \frac{H \mathbf{C} H^{\mathrm{H}}}{K}\right] \mathbb{E}\left[\frac{1}{K} \operatorname{tr} Q(u) Q\left(\sigma^{2}\right)^{m}\right] \\
& -\sum_{k=1}^{m} \mathbb{E}\left[\frac{1}{K} \operatorname{tr} Q(u) Q\left(\sigma^{2}\right)^{k} \frac{H \mathbf{C} H^{\mathrm{H}}}{K}\right] \mathbb{E}\left[\frac{1}{K} \operatorname{tr} Q\left(\sigma^{2}\right)^{m-k+1}\right] \\
& +\mathcal{O}\left(\sqrt{\frac{1}{u^{2} K^{5}} \operatorname{tr} \mathbf{C C}^{\mathrm{H}}}\right) .
\end{aligned}
$$


Define the following quantities

$$
\begin{aligned}
\gamma_{m}^{\circ}(u, \mathbf{C}) & =\mathbb{E}\left[\frac{1}{K} \operatorname{tr} Q(u) Q\left(\sigma^{2}\right)^{m} \frac{H \mathbf{C} H^{\mathrm{H}}}{K}\right], m=0,1, \ldots \\
\delta_{m}^{\circ}(u) & =\mathbb{E}\left[\frac{1}{K} \operatorname{tr} Q(u) Q\left(\sigma^{2}\right)^{m}\right], m=-1,0,1, \ldots
\end{aligned}
$$

which satisfy the relations

$$
\begin{aligned}
\delta_{-1}^{\circ}\left(\sigma^{2}\right) & =c \\
\gamma_{m}^{\circ}\left(u, \mathbf{I}_{K}\right) & =\mathbb{E}\left[\frac{1}{K} \operatorname{tr} Q(u) Q\left(\sigma^{2}\right)^{m} \frac{H H^{\mathrm{H}}}{K}\right] \\
& =\mathbb{E}\left[\frac{1}{K} \operatorname{tr} Q\left(\sigma^{2}\right)^{m}\right]-u \mathbb{E}\left[\frac{1}{K} \operatorname{tr} Q(u) Q\left(\sigma^{2}\right)^{m}\right] \\
& =\delta_{m-1}^{\circ}\left(\sigma^{2}\right)-u \delta_{m}^{\circ}(u), \quad \forall m .
\end{aligned}
$$

For $m \geq 1$, we also have from the relations in (152)

$$
\gamma_{m}^{\circ}\left(u, \mathbf{I}_{K}\right)=\delta_{m-1}^{\circ}(u)-\sigma^{2} \delta_{m}^{\circ}(u) .
$$

Using these definitions, we can express (309) as

$$
\begin{aligned}
& \gamma_{m}^{\circ}(u, \mathbf{C})=\frac{1}{K} \operatorname{tr} \mathbf{C} \delta_{m}^{\circ}(u)-\gamma_{0}^{\circ}(u, \mathbf{C}) \delta_{m}^{\circ}(u) \\
& -\sum_{k=1}^{m} \gamma_{k}^{\circ}(u, \mathbf{C}) \delta_{m-k}^{\circ}\left(\sigma^{2}\right)+\mathcal{O}\left(\sqrt{\frac{1}{u^{2} K^{5}} \operatorname{tr} \mathbf{C} \mathbf{C}^{\mathrm{H}}}\right) .
\end{aligned}
$$

Evaluating the last equation for $m=0$ and collecting the terms in $\gamma_{0}^{\circ}(u, \mathbf{C})$ on one side, leads to

$$
\gamma_{0}^{\circ}(u, \mathbf{C})=\frac{\delta_{0}^{\circ}(u)}{1+\delta_{0}^{\circ}(u)} \frac{1}{K} \operatorname{tr} \mathbf{C}+\mathcal{O}\left(\sqrt{\frac{1}{u^{2} K^{5}} \operatorname{tr} \mathbf{C} \mathbf{C}^{\mathrm{H}}}\right) .
$$

By Theorem 4 in Appendix C.

$$
\delta_{0}^{\circ}(u)=\delta_{0}(u)+\mathcal{O}\left(\frac{1}{u^{4} K^{2}}\right) .
$$

Thus, we can define

$$
\gamma_{0}(u) \triangleq \frac{\delta_{0}(u)}{1+\delta_{0}(u)}
$$

such that

$$
\gamma_{0}^{\circ}(u, \mathbf{C})=\gamma_{0}(u) \frac{1}{K} \operatorname{tr} \mathbf{C}+\mathcal{O}\left(\sqrt{\frac{1}{u^{2} K^{5}} \operatorname{tr} \mathbf{C C}^{\mathrm{H}}}\right)
$$

where we use the fact that $\left|\frac{1}{K} \operatorname{tr} \mathbf{C}\right| \leq \sqrt{\frac{1}{K^{5}} \operatorname{tr} \mathbf{C C}^{\mathbf{H}}}$ and $u^{-4} \leq$ $u^{-1} \sigma^{6}$ (since $u \geq \sigma^{2}$ ) to discard the term $\mathcal{O}\left(u^{-4} K^{-3} \operatorname{tr} \mathbf{C}\right.$ ).

For $m \geq 1$, we can gather the terms involving $\gamma_{m}^{\circ}(u, \mathbf{C})$ in (317) on one side, replace $\gamma_{0}^{\circ}(u, \mathbf{C})$ by $\gamma_{0}(u) \frac{1}{K} \operatorname{tr} \mathbf{C}$ and $\delta_{0}^{\circ}(u)$ by $\delta_{0}(u)$, to obtain, iteratively on $m$,

$$
\begin{aligned}
\gamma_{m}^{\circ}(u, \mathbf{C})= & \frac{\frac{1}{K} \operatorname{tr} \mathbf{C} \delta_{m}^{\circ}(u)-\gamma_{0}(u) \frac{1}{K} \operatorname{tr} \mathbf{C} \delta_{m}^{\circ}(u)}{1+\delta_{0}\left(\sigma^{2}\right)} \\
& -\frac{\sum_{k=1}^{m-1} \gamma_{k}^{\circ}(u, \mathbf{C}) \delta_{m-k}^{\circ}\left(\sigma^{2}\right)}{1+\delta_{0}\left(\sigma^{2}\right)} \\
& +\mathcal{O}\left(\sqrt{\frac{1}{u^{2} K^{5}} \operatorname{tr} \mathbf{C} \mathbf{C}^{\mathrm{H}}}\right) .
\end{aligned}
$$

From the last equation, we can obtain a recursive expression of $\delta_{m}(u)^{\circ}$ by letting $\mathbf{C}=\mathbf{I}_{K}$ and using the relations (315) and (316):

$$
\begin{aligned}
\delta_{m}^{\circ}(u)= & \frac{\delta_{m-1}^{\circ}(u)\left[1+\delta_{0}\left(\sigma^{2}\right)\right]}{1-c+\sigma^{2}\left[1+\delta_{0}\left(\sigma^{2}\right)\right]+u \delta_{0}(u)} \\
& +\frac{\sum_{k=1}^{m-1}\left[\delta_{k-1}^{\circ}(u)-\sigma^{2} \delta_{k}^{\circ}(u)\right] \delta_{m-k}^{\circ}\left(\sigma^{2}\right)}{1-c+\sigma^{2}\left[1+\delta_{0}\left(\sigma^{2}\right)\right]+u \delta_{0}(u)} \\
& +\mathcal{O}\left(\frac{1}{u K^{2}}\right)
\end{aligned}
$$

Note that the denominator of the RHS of the last equation is strictly positive (see Property 1 (i) $-($ iii $)$ in Appendix C). For $m=1$, we obtain with the help of (319)

$$
\begin{aligned}
\delta_{1}^{\circ}(u) & =\frac{\delta_{0}^{\circ}(u)\left[1+\delta_{0}\left(\sigma^{2}\right)\right]}{1-c+\sigma^{2}\left[1+\delta_{0}\left(\sigma^{2}\right)\right]+u \delta_{0}(u)}+\mathcal{O}\left(\frac{1}{u K^{2}}\right) \\
& =\frac{\delta_{0}\left[1+\delta_{0}(u)\left(\sigma^{2}\right)\right]}{1-c+\sigma^{2}\left[1+\delta_{0}\left(\sigma^{2}\right)\right]+u \delta_{0}(u)}+\mathcal{O}\left(\frac{1}{u K^{2}}\right) .
\end{aligned}
$$

Due to the recursive definition of $\delta_{m}^{\circ}(u)$, we can now conclude that

$$
\delta_{m}^{\circ}(u)=\delta_{m}(u)+\mathcal{O}\left(\frac{1}{u K^{2}}\right)
$$

where

$$
\begin{aligned}
\delta_{m}(u)= & \frac{\delta_{m-1}(u)\left[1+\delta_{0}\left(\sigma^{2}\right)\right]}{1-c+\sigma^{2}\left[1+\delta_{0}\left(\sigma^{2}\right)\right]+u \delta_{0}(u)} \\
& +\frac{\sum_{k=1}^{m-1}\left[\delta_{k-1}(u)-\sigma^{2} \delta_{k}(u)\right] \delta_{m-k}\left(\sigma^{2}\right)}{1-c+\sigma^{2}\left[1+\delta_{0}\left(\sigma^{2}\right)\right]+u \delta_{0}(u)}, m \geq 1 .
\end{aligned}
$$

Using (326) in 322), we have so far proved that, for $m \geq 1$,

$$
\begin{aligned}
\gamma_{m}^{\circ}(u, \mathbf{C})=\frac{\frac{1}{K} \operatorname{tr} \mathbf{C} \delta_{m}(u)-\gamma_{0}(u) \frac{1}{K} \operatorname{tr} \mathbf{C} \delta_{m}(u)}{1+\delta_{0}\left(\sigma^{2}\right)} \\
-\frac{\sum_{k=1}^{m-1} \gamma_{k}^{\circ}(u, \mathbf{C}) \delta_{m-k}\left(\sigma^{2}\right)}{1+\delta_{0}\left(\sigma^{2}\right)} \\
+\mathcal{O}\left(\sqrt{\frac{1}{u^{2} K^{5}} \operatorname{tr} \mathbf{C C}^{\mathrm{H}}}\right)
\end{aligned}
$$

where we have relied on the fact that $\gamma_{k}^{\circ}(u, \mathbf{C}) \frac{1}{u K^{2}} \leq$ $\frac{1}{u^{2} K^{3}} \operatorname{tr} \mathbf{C}=\mathcal{O}\left(\sqrt{\frac{1}{u^{2} K^{5}} \operatorname{tr} \mathbf{C C}^{\mathbf{H}}}\right)$. In particular, for $m=1$, we obtain

$$
\begin{aligned}
\gamma_{1}^{\circ}(u, \mathbf{C})=\frac{\delta_{1}(u)-\gamma_{0}(u) \delta_{1}(u)}{1+\delta_{0}\left(\sigma^{2}\right)} \frac{1}{K} \operatorname{tr} \mathbf{C} \\
+\mathcal{O}\left(\sqrt{\frac{1}{u^{2} K^{5}} \operatorname{tr} \mathbf{C} \mathbf{C}^{\mathrm{H}}}\right) .
\end{aligned}
$$

Iterating the recursion $m-1$ times, we have proved that

$$
\gamma_{m}^{\circ}(u, \mathbf{C})=\gamma_{m}(u) \frac{1}{K} \operatorname{tr} \mathbf{C}+\mathcal{O}\left(\sqrt{\frac{1}{u^{2} K^{5}} \operatorname{tr} \mathbf{C} \mathbf{C}^{\mathrm{H}}}\right)
$$


where, for $m \geq 1$,

$$
\gamma_{t}(u)=\frac{\delta_{m}(u)\left(1-\gamma_{0}(u)\right)-\sum_{k=1}^{m-1} \gamma_{k}(u) \delta_{m-k}\left(\sigma^{2}\right)}{1+\delta_{0}\left(\sigma^{2}\right)} .
$$

Using now the relation $\gamma_{0}(u)=c-u \delta_{0}(u)$ (see Property 1 (iv) in Appendix [C], we write the last equation as

$$
\begin{aligned}
\gamma_{m}(u)\left(1+\delta_{0}\left(\sigma^{2}\right)\right)= & \delta_{m}(u)\left(1-c+u \delta_{0}(u)\right) \\
& -\sum_{k=1}^{m-1} \gamma_{k}(u) \delta_{m-k}\left(\sigma^{2}\right) .
\end{aligned}
$$

Adding $\delta_{m}(u) \sigma^{2}\left[1+\delta_{0}\left(\sigma^{2}\right)\right]$ to both sides, we can express $\delta_{m}(u)$ as

$$
\begin{aligned}
\delta_{m}(u)=\frac{\left[\gamma_{m}(u)+\sigma^{2} \delta_{m}(u)\right]\left[1+\delta_{0}\left(\sigma^{2}\right)\right]}{1-c+\sigma^{2}\left[1+\delta_{0}\left(\sigma^{2}\right)\right]+u \delta_{0}(u)} \\
+\frac{\sum_{k=1}^{m-1} \gamma_{k}(u) \delta_{m-k}\left(\sigma^{2}\right)}{1-c+\sigma^{2}\left[1+\delta_{0}\left(\sigma^{2}\right)\right]+u \delta_{0}(u)} .
\end{aligned}
$$

Equating (333) and (327), we can see that $\gamma_{m}(u)$ must satisfy the following relation

$$
\gamma_{m}(u)=\delta_{m-1}(u)-\sigma^{2} \delta_{m}(u), \quad m \geq 1 .
$$

This terminates the proof.

\section{B. Proof of Proposition 2 in Appendix $D-B$}

We want to derive asymptotically exact approximations of $\mathbb{E}\left[\operatorname{tr} Q(u) \frac{H H^{\mathrm{H}}}{K} e^{\gamma_{n}^{\mathbf{X}^{n}, H^{n}}}\right] \quad($ part $(i))$ and $\mathbb{E}\left[\operatorname{tr} Q\left(\sigma^{2}\right) \frac{H \mathbf{A} H^{\mathrm{H}}}{K} e^{\gamma_{n}^{\mathbf{x}^{n}, H^{n}}}\right]$ (part $\left.(i i)\right)$.

In the proofs below, we will often use the notation $P(t)$ or $P_{i}(t)$ to refer to some non-zero polynomials in $t$ with nonnegative coefficients. These polynomials may take different values from one equation to the next.

Proof of part $(i)$ : By the product rule of differentiation, Lemma 6 and Lemma 8 in Appendix C, we obtain the chain of equations (335)-(338) on the top of the next page.

Gathering the terms involving $\operatorname{tr} Q(u) \frac{H H^{\mathrm{H}}}{K}$ on the LHS yields

$$
\begin{aligned}
\mathbb{E} & {\left[\operatorname{tr} Q(u) \frac{H H^{\mathrm{H}}}{K}\left(1+\frac{1}{u}+\frac{1}{K} \operatorname{tr} Q(u)\right) e^{\gamma_{n}^{\mathbf{x}^{n}, H^{n}}}\right] } \\
= & \frac{N}{u} \mathbb{E}\left[e^{\gamma_{n}^{\mathbf{x}^{n}, H^{n}}}\right] \\
& +\frac{1}{K} \sum_{i, j} \mathbb{E}\left[\frac{\partial \gamma_{n}^{\mathbf{X}^{n}, H^{n}}}{\partial H_{i j}^{*}}\left[H^{\mathrm{H}} Q(u)\right]_{j i} e^{\gamma_{n}^{\mathbf{x}^{n}, H^{n}}}\right] .
\end{aligned}
$$

Recall that $\gamma_{n}^{\mathbf{X}^{n}, H^{n}}=\mathbf{i} t \mu_{n}^{\mathbf{X}^{n}, H^{n}}-\frac{t^{2}}{2}\left(\theta_{n}^{\mathbf{X}^{n}, H^{n}}\right)^{2}+$ $\mathbf{i} \frac{t^{3}}{3} \kappa_{n}^{\mathbf{X}^{n}, H^{n}}$ (243). From the standard derivation rules as provided in Lemma 8 and Corollary 1 in Appendix [C, denoting
$Q=Q\left(\sigma^{2}\right)$ for brevity,

$$
\begin{aligned}
\frac{\partial \mu_{n}^{\mathbf{X}^{n}, H^{n}}}{\partial H_{i j}^{*}}= & \sqrt{\frac{n}{K^{3}}}[Q H]_{i j}-\frac{n+1}{\sqrt{n K^{3}}}[Q H A]_{i j} \\
& +\frac{n+1}{\sqrt{n K^{5}}}\left[Q H \mathbf{A} H^{\mathrm{H}} Q H\right]_{i j} \\
=- & \frac{1}{\sqrt{n K^{3}}}[Q H]_{i j}+\frac{1}{\sqrt{n K^{3}}}\left[Q H \mathbf{X X}^{\mathrm{H}}\right]_{i j} \\
& +\frac{n+1}{\sqrt{n K^{5}}}\left[Q H \mathbf{A} H^{\mathrm{H}} Q H\right]_{i j} .
\end{aligned}
$$

Similarly,

$$
\begin{aligned}
\frac{\partial\left(\theta_{n}^{\mathbf{X}^{n}, H^{n}}\right)^{2}}{\partial H_{i j}^{*}} & -\frac{2(n+1)}{n K}\left[\left(Q \frac{1}{K} H H^{\mathrm{H}}\right)^{2} Q \frac{1}{K} H\right]_{i j} \\
& +\frac{2(n+1)}{n K}\left[Q \frac{1}{K} H H^{\mathrm{H}} Q \frac{1}{K} H\right]_{i j} \\
& -\frac{2 \sigma^{2}}{n K}\left[Q \frac{1}{K} H \mathbf{X X}^{\mathrm{H}} H^{\mathrm{H}} Q^{2} \frac{1}{K} H\right]_{i j} \\
& +\frac{2 \sigma^{2}}{n K}\left[Q^{2} \frac{1}{K} H \mathbf{X} \mathbf{X}^{\mathrm{H}}\right]_{i j} \\
& -\frac{2 \sigma^{2}}{n K}\left[Q^{2} \frac{1}{K} H \mathbf{X X}^{\mathrm{H}} H^{\mathrm{H}} Q \frac{1}{K} H\right]_{i j} \\
= & \frac{2 \sigma^{2}(n+1)}{n K}\left[Q \frac{1}{K} H H^{\mathrm{H}} Q^{2} \frac{1}{K} H\right]_{i j} \\
& +\frac{2 \sigma^{4}}{n K}\left[Q^{2} \frac{1}{K} H \mathbf{X X}^{\mathrm{H}} \tilde{Q}\right]_{i j} \\
& -\frac{2 \sigma^{2}}{K}\left[Q \frac{1}{K n} H \mathbf{X X}^{\mathrm{H}} H^{\mathrm{H}} Q^{2} \frac{1}{K} H\right]_{i j}
\end{aligned}
$$

where, in the last equality, we used $\mathbf{I}_{N}-\frac{1}{K} Q H H^{\mathrm{H}}=\sigma^{2} Q$, $\mathbf{I}_{K}-\frac{1}{K} H^{\mathrm{H}} Q H=\sigma^{2} \tilde{Q}$, and $Q H=H \tilde{Q}$. Following the same derivation, we also have

$$
\begin{aligned}
& \frac{\partial \kappa_{n}^{\mathbf{X}^{n}, H^{n}}}{\partial H_{i j}^{*}}= \\
& \frac{3 \sigma^{2}(n+1)}{\sqrt{n^{3} K^{3}}}\left[\left(Q \frac{1}{K} H H^{\mathrm{H}}\right)^{2} Q^{2} \frac{1}{K} H\right]_{i j} \\
& +\frac{3 \sigma^{4}}{\sqrt{n^{3} K^{3}}}\left[Q \frac{1}{K} H \mathbf{X} \mathbf{X}^{\mathrm{H}} H^{\mathrm{H}} Q^{3} \frac{1}{K} H\right]_{i j} \\
& +\frac{3 \sigma^{4}}{\sqrt{n^{3} K^{3}}}\left[\frac{1}{K} H H^{\mathrm{H}} Q^{3} \frac{1}{K} H \mathbf{X X}^{\mathrm{H}} \tilde{Q}\right]_{i j} \\
& -\frac{3 \sigma^{2}}{\sqrt{n^{3} K^{3}}}\left[\frac{1}{K} H H^{\mathrm{H}} Q^{2} \frac{1}{K} H \mathbf{X} \mathbf{X}^{\mathrm{H}} H^{\mathrm{H}} Q^{2} \frac{1}{K} H\right]_{i j} .
\end{aligned}
$$

Using these results, the second term on the RHS of 339 


$$
\begin{aligned}
& \mathbb{E}\left[\operatorname{tr} Q(u) \frac{H H^{\mathrm{H}}}{K} e^{\gamma_{n}^{\mathbf{x}^{n}, H^{n}}}\right] \\
& =\mathbb{E}\left[\frac{1}{K} \sum_{i, j, k} \frac{\partial\left(H_{k j}^{*} Q(u)_{k i} e \gamma_{n}^{\mathbf{X}^{n}, H^{n}}\right)}{\partial H_{i j}^{*}}\right] \\
& =\mathbb{E}\left[\frac{1}{K} \sum_{i, j, k}\left(\delta_{i k} Q(u)_{k i} e^{\gamma_{n}^{\mathbf{x}^{n}, H^{n}}}-\frac{H_{k j}^{*}[Q(u) H]_{k j} Q(u)_{i i}}{K} e^{\gamma_{n}^{\mathbf{x}^{n}, H^{n}}}+H_{k j}^{*} Q(u)_{k i} \frac{\partial \gamma_{n}^{\mathbf{X}^{n}, H^{n}}}{\partial H_{i j}^{*}} e^{\gamma_{n}^{\mathbf{x}^{n}, H^{n}}}\right)\right] \\
& =\mathbb{E}\left[\left(\operatorname{tr} Q(u)-\frac{1}{K} \operatorname{tr} Q(u) \frac{H H^{\mathrm{H}}}{K} \operatorname{tr} Q(u)+\frac{1}{K} \sum_{i, j} \frac{\partial \gamma_{n}^{\mathbf{X}^{n}, H^{n}}}{\partial H_{i j}^{*}}\left[H^{\mathrm{H}} Q(u)\right]_{j i}\right) e^{\gamma_{n}^{\mathbf{x}^{n}, H^{n}}}\right] \\
& =\mathbb{E}\left[\left(\frac{N}{u}-\frac{1}{u} \operatorname{tr} Q(u) \frac{H H^{\mathrm{H}}}{K}-\frac{1}{K} \operatorname{tr} Q(u) \frac{H H^{\mathrm{H}}}{K} \operatorname{tr} Q(u)+\frac{1}{K} \sum_{i, j} \frac{\partial \gamma_{n}^{\mathbf{X}^{n}, H^{n}}}{\partial H_{i j}^{*}}\left[H^{\mathrm{H}} Q(u)\right]_{j i}\right) e^{\left.\gamma_{n}^{\mathbf{x}^{n}, H^{n}}\right]}\right]
\end{aligned}
$$

can be developed as follows:

$$
\begin{aligned}
\frac{1}{K} \sum_{i, j} \mathbb{E}\left[\frac{\partial \gamma_{n}^{\mathbf{X}^{n}, H^{n}}}{\partial H_{i j}^{*}}\left[H^{\mathrm{H}} Q(u)\right]_{j i} e^{\gamma_{n}^{\mathbf{x}^{n}, H^{n}}}\right] \\
\stackrel{(a)}{=} \mathbf{i} t \frac{n+1}{\sqrt{n K}}\left(\mathbb{E}\left[\frac{1}{K} \operatorname{tr} Q \frac{H \mathbf{X X} \mathbf{X}^{\mathrm{H}} H^{\mathrm{H}}}{(n+1) K} Q(u) e^{\gamma_{n}^{\mathbf{x}^{n}, H^{n}}}\right]\right. \\
\left.+\mathbb{E}\left[\frac{1}{K} \operatorname{tr} Q \frac{H \mathbf{A} H^{\mathrm{H}}}{K} Q \frac{H H^{\mathrm{H}}}{K} Q(u) e^{\gamma_{n}^{\mathbf{x}^{n}, H^{n}}}\right]\right) \\
+\mathcal{O}\left(\frac{P(t)}{u K}\right) \\
\stackrel{(b)}{=} \mathbf{i} t \frac{n+1}{\sqrt{n K}}\left(\mathbb{E}\left[\frac{1}{K} \operatorname{tr} Q \frac{H H^{\mathrm{H}}}{K} Q(u) e^{\gamma_{n}^{\mathbf{x}^{n}, H^{n}}}\right]\right. \\
\left.-\mathbb{E}\left[\frac{\sigma^{2}}{K} \operatorname{tr} Q \frac{H \mathbf{A} H^{\mathrm{H}}}{K} Q Q(u) e^{\gamma_{n}^{\mathbf{x}^{n}, H^{n}}}\right]\right)+\mathcal{O}\left(\frac{P(t)}{u K}\right)
\end{aligned}
$$

for some polynomial $P(t)$, where $(a)$ follows from the derivative of $\gamma_{n}^{\mathbf{X}^{n}, H^{n}}$ as developed in (341)-(344) and the observations that all terms resulting from $\left(\theta_{n}^{\mathbf{X}^{n}, H^{n}}\right)^{2}$ and $\kappa_{n}^{\mathbf{X}^{n}, H^{n}}$ are $\mathcal{O}\left((u K)^{-1}\right)$ and $\mathcal{O}\left(u^{-1} K^{-2}\right)$, respectively, and (b) follows from $Q \frac{H H^{H}}{K}=\mathbf{I}_{N}-\sigma^{2} Q$ (see (152) ) and the definition of $\mathbf{A}=\mathbf{I}_{K}-\frac{1}{n+1} \mathbf{X} \mathbf{X}^{\mathrm{H}}$.

Based on Proposition 3 in Appendix E-A and Lemma 5 in Appendix $[$, we find the following estimations:

$$
\begin{aligned}
& \mathbb{E}\left[\frac{1}{K} \operatorname{tr} Q \frac{H H^{\mathrm{H}}}{K} Q(u) e^{\gamma_{n}^{\mathbf{x}^{n}, H^{n}}}\right] \\
& =\mathbb{E}\left[\frac{1}{K} \operatorname{tr} Q(u) Q \frac{H H^{\mathrm{H}}}{K}\right] \mathbb{E}\left[e^{\gamma_{n}^{\mathbf{x}^{n}, H^{n}}}\right]+\mathcal{O}\left(\frac{1}{u K}\right) \\
& \mathbb{E}\left[\frac{\sigma^{2}}{K} \operatorname{tr} Q \frac{H \mathbf{A} H^{\mathrm{H}}}{K} Q Q(u) e^{\gamma_{n}^{\mathbf{x}^{n}, H^{n}}}\right] \\
& =\mathbb{E}\left[\frac{\sigma^{2}}{K} \operatorname{tr} Q(u) Q^{2} \frac{H \mathbf{A} H^{\mathrm{H}}}{K}\right] \mathbb{E}\left[e^{\gamma_{n}^{\mathbf{x}^{n}, H^{n}}}\right] \\
& \quad+\mathcal{O}\left(\sqrt{\frac{1}{u^{2} K^{3}} \operatorname{tr} \mathbf{A}^{2}}\right) .
\end{aligned}
$$

By Proposition 4 in Appendix E-A

$$
\begin{aligned}
& \mathbb{E}\left[\frac{1}{K} \operatorname{tr} Q(u) Q \frac{H H^{\mathrm{H}}}{K}\right]=\delta_{0}(u)-\sigma^{2} \delta_{1}(u)+\mathcal{O}\left(\frac{1}{u K^{2}}\right) \\
& \mathbb{E}\left[\frac{\sigma^{2}}{K} \operatorname{tr} Q(u) Q^{2} \frac{H \mathbf{A} H^{\mathrm{H}}}{K}\right] \\
& =\sigma^{2} \gamma_{2}(u) \frac{1}{K} \operatorname{tr} \mathbf{A}+\mathcal{O}\left(\sqrt{\frac{1}{u^{2} K^{5}} \operatorname{tr} \mathbf{A}^{2}}\right) \\
& =\mathcal{O}\left(\sqrt{\frac{1}{u^{2} K^{5}} \operatorname{tr} \mathbf{A}^{2}}\right)
\end{aligned}
$$

Combining (339), (346), 347), (348), 349), and (351), we obtain

$$
\begin{aligned}
\mathbb{E} & {\left[\operatorname{tr} Q(u) \frac{H H^{\mathrm{H}}}{K}\left(1+\frac{1}{u}+\frac{1}{K} \operatorname{tr} Q(u)\right) e^{\gamma_{n}^{\mathbf{x}^{n}, H^{n}}}\right] } \\
= & \frac{N}{u} \mathbb{E}\left[e^{\gamma_{n}^{\mathbf{x}^{n}, H^{n}}}\right]+\mathcal{O}\left(\frac{1}{u \sqrt{K}} P(t)\right) \\
& +\mathbf{i} t \frac{n+1}{\sqrt{n K}}\left(\delta_{0}(u)-\sigma^{2} \delta_{1}(u)\right) \mathbb{E}\left[e^{\gamma_{n}^{\mathbf{x}^{n}, H^{n}}}\right] \\
= & \frac{N}{u} \mathbb{E}\left[e^{\gamma_{n}^{\mathbf{x}^{n}, H^{n}}}\right]+\mathbf{i} t \sqrt{\frac{n}{K}}\left(\delta_{0}(u)-\sigma^{2} \delta_{1}(u)\right) \mathbb{E}\left[e^{\gamma_{n}^{\mathbf{x}^{n}, H^{n}}}\right] \\
& +\mathcal{O}\left(\frac{1}{u \sqrt{K}} P(t)\right)
\end{aligned}
$$

for some other polynomial $P(t)$, where we used in particular $\sqrt{K^{-3} \operatorname{tr} \mathbf{A}^{2}} \leq 1 / \sqrt{K}$ and $\frac{1}{\sqrt{n K}}\left(\delta_{0}(u)-\sigma^{2} \delta_{1}(u)\right)=$ $\mathcal{O}\left((u K)^{-1}\right)$ by Property 1 in Appendix $\mathrm{C}$

Next, we consider the LHS of (339). Let us first define the following quantities:

$$
\Psi=\frac{1}{K} \operatorname{tr} Q(u), \quad \Phi=\operatorname{tr} Q(u) \frac{H H^{\mathrm{H}}}{K} .
$$

Using these definitions, we can express the LHS of (339) as

$$
\begin{aligned}
& \mathbb{E}\left[\operatorname{tr} Q(u) \frac{H H^{\mathrm{H}}}{K}\left(1+\frac{1}{u}+\frac{1}{K} \operatorname{tr} Q(u)\right) e^{\gamma_{n}^{\mathbf{x}^{n}, H^{n}}}\right] \\
& =\left(1+\frac{1}{u}\right) \mathbb{E}\left[\Phi e^{\gamma_{n}^{\mathbf{x}^{n}, H^{n}}}\right]+\mathbb{E}\left[\Phi \Psi e^{\gamma_{n}^{\mathbf{x}^{n}, H^{n}}}\right] .
\end{aligned}
$$


We can now develop the second term on the RHS of the last equation as follows:

$$
\begin{aligned}
& \mathbb{E}\left[\Phi \Psi e^{\gamma_{n}^{\mathbf{x}^{n}, H^{n}}}\right] \\
& =\mathbb{E}[\Psi] \mathbb{E}\left[\Phi e^{\gamma_{n}^{\mathbf{X}^{n}, H^{n}}}\right]+\mathbb{E}\left[\Phi(\Psi-\mathbb{E}[\Psi]) e^{\gamma_{n}^{\mathbf{X}^{n}, H^{n}}}\right] \\
& \stackrel{(a)}{=} \mathbb{E}[\Psi] \mathbb{E}\left[\Phi e^{\gamma_{n}^{\mathbf{x}^{n}, H^{n}}}\right]-\mathbb{E}[\Phi] \mathbb{E}[\Psi] \mathbb{E}\left[e^{\gamma_{n}^{\mathbf{x}^{n}, H^{n}}}\right] \\
& +\mathbb{E}[\Phi] \mathbb{E}\left[\left(\frac{1}{u} \frac{N}{K}-\frac{1}{u} \frac{1}{K} \operatorname{tr} Q(u) \frac{H H^{\mathbf{H}}}{K}\right) e^{\gamma_{n}^{\mathbf{x}^{n}, H^{n}}}\right] \\
& +\mathcal{O}\left(\frac{1}{u^{2} K}\right) \\
& =\mathbb{E}[\Psi] \mathbb{E}\left[\Phi e^{\gamma_{n}^{\mathbf{X}^{n}, H^{n}}}\right]-\mathbb{E}[\Phi] \mathbb{E}[\Psi] \mathbb{E}\left[e^{\gamma_{n}^{\mathbf{X}^{n}, H^{n}}}\right] \\
& +\frac{1}{u} \frac{N}{K} \mathbb{E}[\Phi] \mathbb{E}\left[e^{\gamma_{n}^{\mathbf{x}^{n}, H^{n}}}\right]-\frac{1}{u} \mathbb{E}\left[\frac{1}{K} \Phi\right] \mathbb{E}\left[\Phi e^{\gamma_{n}^{\mathbf{x}^{n}, H^{n}}}\right] \\
& +\mathcal{O}\left(\frac{1}{u^{2} K}\right) \\
& =\mathbb{E}\left[\Phi e^{\gamma_{n}^{\mathbf{x}^{n}, H^{n}}}\right]\left(\mathbb{E}[\Psi]-\frac{1}{u} \mathbb{E}\left[\frac{1}{K} \Phi\right]\right) \\
& -\mathbb{E}[\Phi] \mathbb{E}[\Psi] \mathbb{E}\left[e^{\gamma_{n}^{\mathbf{x}^{n}, H^{n}}}\right]+\frac{1}{u} \frac{N}{K} \mathbb{E}[\Phi] \mathbb{E}\left[e^{\gamma_{n}^{\mathbf{x}^{n}, H^{n}}}\right] \\
& +\mathcal{O}\left(\frac{1}{u^{2} K}\right) \\
& \stackrel{(b)}{=} \mathbb{E}\left[\Phi e^{\gamma_{n}^{\mathbf{x}^{n}, H^{n}}}\right]\left(\delta_{0}(u)-\frac{1}{u} \gamma_{0}(u)\right) \\
& -K \gamma_{0}(u) \delta_{0}(u) \mathbb{E}\left[e^{\gamma_{n}^{\mathbf{x}^{n}, H^{n}}}\right]+\frac{1}{u} c K \gamma_{0}(u) \mathbb{E}\left[e^{\gamma_{n}^{\mathbf{x}^{n}, H^{n}}}\right] \\
& +\mathcal{O}\left(\frac{1}{u K}\right) \\
& \stackrel{(c)}{=}\left(2 \delta_{0}(u)-\frac{c}{u}\right) \mathbb{E}\left[\Phi e^{\gamma_{n}^{\mathbf{X}^{n}, H^{n}}}\right] \\
& +N\left(\frac{c}{u}-2 \delta_{0}(u)+\frac{u}{c} \delta_{0}(u)^{2}\right) \mathbb{E}\left[e^{\gamma_{n}^{\mathbf{x}^{n}, H^{n}}}\right] \\
& +\mathcal{O}\left(\frac{1}{u K}\right)
\end{aligned}
$$

where $(a)$ follows from Remark 7 and Proposition 3 in Appendix E-A, and $\Psi$ is expanded using (152), (b) follows by Proposition 4 in Appendix E-A and the fact that $\left|e^{\gamma_{n}^{\mathbf{x}^{n}, H^{n}}}\right| \leq$ 1 , and in $(c)$ we used $\gamma_{0}(u)=c-u \delta_{0}(u)$ (see Proposition 4). Thus, 355 can be expressed as

$$
\begin{aligned}
& \mathbb{E}\left[\Phi\left(1+\frac{1}{u}+\Psi\right) e^{\gamma_{n}^{\mathbf{x}^{n}, H^{n}}}\right] \\
& =\left(1+\frac{1-c}{u}+2 \delta_{0}(u)\right) \mathbb{E}\left[\Phi e^{\gamma_{n}^{\mathbf{x}^{n}, H^{n}}}\right] \\
& \quad+N\left(\frac{c}{u}-2 \delta_{0}(u)+\frac{u}{c} \delta_{0}(u)^{2}\right) \mathbb{E}\left[e^{\gamma_{n}^{\mathbf{x}^{n}, H^{n}}}\right] \\
& \quad+\mathcal{O}\left(\frac{1}{u K}\right) .
\end{aligned}
$$

Equating the RHS of (362) and the RHS of (353) and solving for $\mathbb{E}\left[\Phi e^{\gamma_{n}^{\mathbf{x}^{n}, H^{n}}}\right]$ leads to

$$
\begin{aligned}
\mathbb{E}\left[\Phi e^{\gamma_{n}^{\mathbf{x}^{n}, H^{n}}}\right] \\
=N \frac{\left(1-c+2 u \delta_{0}(u)-\frac{u^{2}}{c} \delta_{0}(u)^{2}\right)}{1-c+u\left(1+2 \delta_{0}(u)\right)} \mathbb{E}\left[e^{\gamma_{n}^{\mathbf{x}^{n}, H^{n}}}\right] \\
\quad+\mathbf{i} t \sqrt{\frac{n}{K}} u \frac{\delta_{0}(u)-\sigma^{2} \delta_{1}(u)}{1-c+u\left(1+2 \delta_{0}(u)\right)} \mathbb{E}\left[e^{\gamma_{n}^{\mathbf{x}^{n}, H^{n}}}\right] \\
+\mathcal{O}\left(\frac{1}{u \sqrt{K}} P(t)\right)
\end{aligned}
$$

for some polynomial $P(t)$.

This concludes the proof of part $(i)$.

Proof of part $(i i)$ : We begin as in the proof of part $(i)$. From the derivative of $\gamma_{n}^{\mathbf{X}^{n}, H^{n}}$ in 341 344 and standard Gaussian calculus, we have

$$
\begin{aligned}
\mathbb{E} & {\left[\operatorname{tr} Q \frac{H \mathbf{A} H^{\mathrm{H}}}{K} e^{\gamma_{n}^{\mathbf{x}^{n}, H^{n}}}\right] } \\
= & \frac{1}{K} \sum_{i, j, k, l} \mathbb{E}\left[H_{i j} A_{j k} H_{l k}^{*} Q_{l i} e^{\gamma_{n}^{\mathbf{x}^{n}, H^{n}}}\right] \\
= & \frac{1}{K} \sum_{i, j, k, l} A_{j k} \mathbb{E}\left[\frac{\partial\left(H_{l k}^{*} Q_{l i} e^{\gamma_{n}^{\mathbf{x}^{n}, H^{n}}}\right)}{\partial H_{i j}^{*}}\right] \\
= & \frac{1}{K} \operatorname{tr} \mathbf{A} \mathbb{E}\left[\operatorname{tr} Q e^{\left.\gamma_{n}^{\mathbf{x}^{n}, H^{n}}\right]}-\mathbb{E}\left[\frac{1}{K} \operatorname{tr} Q \operatorname{tr} Q \frac{H \mathbf{A} H^{\mathrm{H}}}{K} e^{\gamma_{n}^{\mathbf{x}^{n}, H^{n}}}\right]\right. \\
& +\frac{1}{K} \sum_{i, j} \mathbb{E}\left[\left[\mathbf{A} H^{\mathrm{H}} Q\right]_{j i} \frac{\partial \gamma_{n}^{\mathbf{X}^{n}, H^{n}}}{\partial H_{i j}^{*}} e^{\left.\gamma_{n}^{\mathbf{x}^{n}, H^{n}}\right]}\right. \\
= & -\mathbb{E}\left[\frac{1}{K} \operatorname{tr} Q \operatorname{tr} Q \frac{H \mathbf{A} H^{\mathrm{H}}}{K} e^{\left.\gamma_{n}^{\mathbf{x}^{n}, H^{n}}\right]}\right] \\
& +\mathrm{i} t \sqrt{\frac{n}{K}} \mathbb{E}\left[\left(\frac{1}{K} \operatorname{tr} Q^{2} \frac{H \mathbf{X} \mathbf{X}^{\mathrm{H}} \mathbf{A} H^{\mathrm{H}}}{K(n+1)}\right.\right. \\
& \left.+\frac{1}{K} \operatorname{tr} Q\left(\frac{Q H \mathbf{A} H^{\mathrm{H}}}{K}\right) e^{2} e_{n}^{\mathbf{x}^{n}, H^{n}}\right] \\
+ & \mathcal{O}\left(\frac{P_{1}(t)}{K}\left(1+\frac{1}{K} \operatorname{tr} \mathbf{A}^{2}\right)\right)
\end{aligned}
$$

for some polynomial $P_{1}(t)$, where the last line follows from the observation that $\operatorname{tr} \mathbf{A}=0$ and that the terms in the derivative of $\gamma_{n}^{\mathbf{X}^{n}, H^{n}}$ resulting from $\left(\theta_{n}^{\mathbf{X}^{n}, H^{n}}\right)^{2}$ and $\kappa_{n}^{\mathbf{X}^{n}, H^{n}}$ are of order $\mathcal{O}\left(\frac{t^{2}}{K}\left(1+\frac{1}{K} \operatorname{tr} \mathbf{A}^{2}\right)\right)$ and $\mathcal{O}\left(\frac{t^{3}}{K^{2}}\left(1+\frac{1}{K} \operatorname{tr} \mathbf{A}^{2}\right)\right)$, respectively.

Rearranging the terms, one arrives at

$$
\begin{aligned}
\mathbb{E}\left[\operatorname{tr} Q \frac{H \mathbf{A} H^{\mathrm{H}}}{K}\left(1+\frac{1}{K} \operatorname{tr} Q\right) e^{\gamma_{n}^{\mathbf{x}^{n}, H^{n}}}\right] \\
=\mathbf{i} t \sqrt{\frac{n}{K}} \mathbb{E}\left[\left(\frac{1}{K} \operatorname{tr} Q^{2} \frac{H \mathbf{X} \mathbf{X}^{\mathrm{H}} \mathbf{A} H^{\mathrm{H}}}{K(n+1)}\right.\right. \\
\left.\left.+\frac{1}{K} \operatorname{tr} Q\left(Q \frac{H \mathbf{A} H^{\mathrm{H}}}{K}\right)^{2}\right) e^{\gamma_{n}^{\mathbf{x}^{n}, H^{n}}}\right] \\
+\mathcal{O}\left(\frac{P_{1}(t)}{K}\left(1+\frac{1}{K} \operatorname{tr} \mathbf{A}^{2}\right)\right) .
\end{aligned}
$$


Using the identity $\mathbf{A}-\mathbf{A}^{2}=\frac{\mathbf{x X}^{\mathrm{H}}}{n+1} \mathbf{A}$, we obtain

$$
\begin{aligned}
& \mathbf{i} t \sqrt{\frac{n}{K}} \mathbb{E}\left[\left(\frac{1}{K} \operatorname{tr} Q^{2} \frac{H \mathbf{X} \mathbf{X}^{\mathrm{H}} \mathbf{A} H^{\mathrm{H}}}{K(n+1)}\right.\right. \\
& \left.+\frac{1}{K} \operatorname{tr} Q\left(Q \frac{H \mathbf{A} H^{\mathrm{H}}}{K}\right)^{2}\right) e^{\left.\gamma_{n}^{\mathbf{x}^{n}, H^{n}}\right]} \\
& =\mathbf{i} t \sqrt{\frac{n}{K}} \mathbb{E}\left[\left(\frac{1}{K} \operatorname{tr} Q^{2} \frac{H \mathbf{A} H^{\mathrm{H}}}{K}-\frac{1}{K} \operatorname{tr} Q^{2} \frac{H \mathbf{A}^{2} H^{\mathrm{H}}}{K}\right.\right. \\
& \left.+\frac{1}{K} \operatorname{tr} Q\left(Q \frac{H \mathbf{A} H^{\mathrm{H}}}{K}\right)^{2}\right) e^{\left.\gamma_{n}^{\mathbf{x}^{n}, H^{n}}\right] .}
\end{aligned}
$$

Note now that

$$
\begin{aligned}
& \operatorname{Var}\left[\frac{1}{K} \operatorname{tr} Q\left(Q \frac{H \mathbf{A} H^{\mathrm{H}}}{K}\right)^{2}\right] \\
&=\operatorname{Var}\left[\frac{1}{K} \operatorname{tr} \tilde{Q}^{2} \frac{H^{\mathrm{H}} H}{K} \mathbf{A} \tilde{Q} \frac{H^{\mathrm{H}} H}{K} \mathbf{A}\right] \\
&=\operatorname{Var}\left[\frac{1}{K} \operatorname{tr} \tilde{Q}\left(\mathbf{I}_{K}-\sigma^{2} \tilde{Q}\right) \mathbf{A}\left(\mathbf{I}_{K}-\sigma^{2} \tilde{Q}\right) \mathbf{A}\right] \\
& \leq\left(\sqrt{\operatorname{Var}\left[\frac{1}{K} \operatorname{tr} \tilde{Q} \mathbf{A}^{2}\right]}+\sqrt{\mathbb{V a r}\left[\frac{\sigma^{2}}{K} \operatorname{tr} \tilde{Q}^{2} \mathbf{A}^{2}\right]}\right. \\
&\left.+\sqrt{\operatorname{Var}\left[\frac{\sigma^{2}}{K} \operatorname{tr}(\tilde{Q} \mathbf{A})^{2}\right]}+\sqrt{\mathbb{V a r}\left[\frac{\sigma^{4}}{K} \operatorname{tr} \tilde{Q}^{2} \mathbf{A} \tilde{Q} \mathbf{A}\right]}\right)^{2} \\
&= \mathcal{O}\left(\frac{1}{K^{3}} \operatorname{tr} \mathbf{A}^{4}\right)=\mathcal{O}\left(\frac{1}{K}\left(\frac{1}{K} \operatorname{tr} \mathbf{A}^{2}\right)^{2}\right)
\end{aligned}
$$

where the inequality follows from Remark 7 in Appendix C and the last line follows from a direct application of Proposition 3 in Appendix E-A to each of the individual terms, along with $\operatorname{tr} \mathbf{A}^{4} \leq\left(\operatorname{tr} \mathbf{A}^{2}\right)^{2}$. By Proposition 3 ,

$$
\begin{aligned}
\operatorname{Var}\left[\frac{1}{K} \operatorname{tr} Q^{2} \frac{H \mathbf{A} H^{\mathrm{H}}}{K}\right] & =\mathcal{O}\left(\frac{1}{K^{3}} \operatorname{tr} \mathbf{A}^{2}\right)=\mathcal{O}\left(\frac{1}{K}\right) \\
\operatorname{Var}\left[\frac{1}{K} \operatorname{tr} Q^{2} \frac{H \mathbf{A}^{2} H^{\mathrm{H}}}{K}\right] & =\mathcal{O}\left(\frac{1}{K^{3}} \operatorname{tr} \mathbf{A}^{4}\right) \\
& =\mathcal{O}\left(\frac{1}{K}\left(\frac{1}{K} \operatorname{tr} \mathbf{A}^{2}\right)^{2}\right)
\end{aligned}
$$

Thus, by Lemma 5 in Appendix C, the RHS of (369) can be written as in (377) on the top of the next page. By Proposition 4 in Appendix E-A, we can approximate the first two terms in 377 by

$$
\begin{aligned}
& \mathbb{E}\left[\frac{1}{K} \operatorname{tr} Q^{2} \frac{H \mathbf{A} H^{\mathrm{H}}}{K}\right] \\
& =\gamma_{1}\left(\sigma^{2}\right) \frac{1}{K} \operatorname{tr} \mathbf{A}+\mathcal{O}\left(\sqrt{\frac{1}{K^{5}} \operatorname{tr} \mathbf{A}^{2}}\right) \\
& =\mathcal{O}\left(\frac{1}{\sqrt{K^{3}}}\right) \\
& \mathbb{E}\left[\frac{1}{K} \operatorname{tr} Q^{2} \frac{H \mathbf{A}^{2} H^{\mathrm{H}}}{K}\right] \\
& =\gamma_{1}\left(\sigma^{2}\right) \frac{1}{K} \operatorname{tr} \mathbf{A}^{2}+\mathcal{O}\left(\sqrt{\frac{1}{K^{5}}} \operatorname{tr} \mathbf{A}^{4}\right) \\
& =\gamma_{1}\left(\sigma^{2}\right) \frac{1}{K} \operatorname{tr} \mathbf{A}^{2}+\mathcal{O}\left(\frac{1}{\sqrt{K^{3}}} \frac{1}{K} \operatorname{tr} \mathbf{A}^{2}\right) .
\end{aligned}
$$

It remains to find an approximation of the term $\mathbb{E}\left[\frac{1}{K} \operatorname{tr} Q\left(Q \frac{H \mathbf{A} H^{\mathrm{H}}}{K}\right)^{2}\right]$. By Lemma 6 in Appendix $\mathbb{C}$

$$
\begin{aligned}
& \mathbb{E}\left[\frac{1}{K} \operatorname{tr} Q\left(Q \frac{H \mathbf{A} H^{\mathrm{H}}}{K}\right)^{2}\right] \\
& =\mathbb{E}\left[\frac{1}{K^{3}} \operatorname{tr} H \mathbf{A} H^{\mathrm{H}} Q^{2} H \mathbf{A} H^{\mathrm{H}} Q\right] \\
& =\frac{1}{K^{3}} \sum_{i, j} \mathbb{E}\left[H_{i j}\left[\mathbf{A} H^{\mathrm{H}} Q^{2} H \mathbf{A} H^{\mathrm{H}} Q\right]_{j i}\right] \\
& =\frac{1}{K^{3}} \sum_{i, j} \mathbb{E}\left[\frac{\partial\left[\mathbf{A} H^{\mathrm{H}} Q^{2} H \mathbf{A} H^{\mathrm{H}} Q\right]_{j i}}{\partial H_{i j}^{*}}\right] .
\end{aligned}
$$

The derivative further develops as

$$
\begin{aligned}
& \frac{\partial\left[\mathbf{A} H^{\mathrm{H}} Q^{2} H \mathbf{A} H^{\mathrm{H}} Q\right]_{j i}}{\partial H_{i j}^{*}}= \\
& A_{j j}\left[Q^{2} H \mathbf{A} H^{\mathrm{H}} Q\right]_{i i}-\frac{1}{K}\left[\mathbf{A} H^{\mathrm{H}} Q H\right]_{j j}\left[Q^{2} H \mathbf{A} H^{\mathrm{H}} Q\right]_{i i} \\
& -\frac{1}{K}\left[\mathbf{A} H^{\mathrm{H}} Q^{2} H\right]_{j j}\left[Q H \mathbf{A} H^{\mathrm{H}} Q\right]_{i i}+\left[\mathbf{A} H^{\mathrm{H}} Q^{2} H \mathbf{A}\right]_{j j} Q_{i i} \\
& -\frac{1}{K}\left[\mathbf{A} H^{\mathrm{H}} Q^{2} H \mathbf{A} H^{\mathrm{H}} Q H\right]_{j j} Q_{i i} .
\end{aligned}
$$

Replacing 385 in (384) and rearranging the resulting terms, we arrive at

$$
\begin{aligned}
\mathbb{E} & {\left[\frac{1}{K} \operatorname{tr} Q\left(Q \frac{H \mathbf{A} H^{\mathrm{H}}}{K}\right)^{2}\left(1+\frac{1}{K} \operatorname{tr} Q\right)\right] } \\
= & \mathbb{E}\left[\frac{1}{K} \operatorname{tr} Q^{3} \frac{H \mathbf{A} H^{\mathrm{H}}}{K}\left(\frac{1}{K} \operatorname{tr} \mathbf{A}-\frac{1}{K} \operatorname{tr} Q \frac{H \mathbf{A} H^{\mathrm{H}}}{K}\right)\right] \\
- & \mathbb{E}\left[\left(\frac{1}{K} \operatorname{tr} Q^{2} \frac{H \mathbf{A} H^{\mathrm{H}}}{K}\right)^{2}\right] \\
+ & \mathbb{E}\left[\frac{1}{K} \operatorname{tr} Q \frac{1}{K} \operatorname{tr} Q^{2} \frac{H \mathbf{A}^{2} H^{\mathrm{H}}}{K}\right] .
\end{aligned}
$$

Applying Proposition 4 in Appendix E-A together with Proposition 3 in Appendix E-A and Lemma 5 in Appendix $\mathrm{C}$ 


$$
\begin{aligned}
& \mathbf{i} t \sqrt{\frac{n}{K}} \mathbb{E}\left[\left(\frac{1}{K} \operatorname{tr} Q^{2} \frac{H \mathbf{A} H^{\mathrm{H}}}{K}-\frac{1}{K} \operatorname{tr} Q^{2} \frac{H \mathbf{A}^{2} H^{\mathrm{H}}}{K}+\frac{1}{K} \operatorname{tr} Q\left(Q \frac{H \mathbf{A} H^{\mathrm{H}}}{K}\right)^{2}\right) e^{\gamma_{n}^{\mathbf{x}^{n}, H^{n}}}\right] \\
& =\mathbf{i} t \sqrt{\frac{n}{K}} \mathbb{E}\left[\left(\frac{1}{K} \operatorname{tr} Q^{2} \frac{H \mathbf{A} H^{\mathrm{H}}}{K}-\frac{1}{K} \operatorname{tr} Q^{2} \frac{H \mathbf{A}^{2} H^{\mathrm{H}}}{K}+\frac{1}{K} \operatorname{tr} Q\left(Q \frac{H \mathbf{A} H^{\mathrm{H}}}{K}\right)^{2}\right)\right] \mathbb{E}\left[e^{\gamma_{n}^{\mathbf{x}^{n}, H^{n}}}\right]+\mathcal{O}\left(\frac{t}{\sqrt{K}}\left(1+\frac{1}{K} \operatorname{tr} \mathbf{A}^{2}\right)\right)
\end{aligned}
$$

to the individual terms leads to

$$
\begin{aligned}
& \mathbb{E}\left[\frac{1}{K} \operatorname{tr} Q\left(Q \frac{H \mathbf{A} H^{\mathrm{H}}}{K}\right)^{2}\left(1+\frac{1}{K} \operatorname{tr} Q\right)\right] \\
& =\delta_{0}\left(\sigma^{2}\right) \gamma_{1}\left(\sigma^{2}\right) \frac{1}{K} \operatorname{tr} \mathbf{A}^{2}+\mathcal{O}\left(\frac{1}{\sqrt{K^{5}}} \operatorname{tr} \mathbf{A}^{2}\right) .
\end{aligned}
$$

Similarly, by Lemma 5, Proposition 3, the variance bound in (373), and Proposition 4

$$
\begin{aligned}
\mathbb{E} & {\left[\frac{1}{K} \operatorname{tr} Q\left(Q \frac{H \mathbf{A} H^{\mathrm{H}}}{K}\right)^{2}\left(1+\frac{1}{K} \operatorname{tr} Q\right)\right] } \\
= & \mathbb{E}\left[\frac{1}{K} \operatorname{tr} Q\left(Q \frac{H \mathbf{A} H^{\mathrm{H}}}{K}\right)^{2}\right] \mathbb{E}\left[1+\frac{1}{K} \operatorname{tr} Q\right] \\
& +\mathcal{O}\left(\sqrt{\frac{1}{K^{5}} \operatorname{tr} \mathbf{A}^{4}}\right) \\
= & \mathbb{E}\left[\frac{1}{K} \operatorname{tr} Q\left(Q \frac{H \mathbf{A} H^{\mathrm{H}}}{K}\right)^{2}\right]\left(1+\delta_{0}\left(\sigma^{2}\right)\right) \\
& +\mathcal{O}\left(\frac{1}{\sqrt{K^{5}}} \operatorname{tr} \mathbf{A}^{2}\right) .
\end{aligned}
$$

Equating the RHSs of 387 and 389 and solving for $\mathbb{E}\left[\frac{1}{K} \operatorname{tr} Q\left(Q \frac{H \mathbf{A} H^{\mathrm{H}}}{K}\right)^{2}\right]$ yields

$$
\begin{aligned}
\mathbb{E}\left[\frac{1}{K} \operatorname{tr} Q\left(Q \frac{H \mathbf{A} H^{\mathrm{H}}}{K}\right)^{2}\right]= & \frac{\delta_{0}\left(\sigma^{2}\right) \gamma_{1}\left(\sigma^{2}\right) \frac{1}{K} \operatorname{tr} \mathbf{A}^{2}}{1+\delta_{0}\left(\sigma^{2}\right)} \\
& +\mathcal{O}\left(\frac{1}{\sqrt{K^{5}}} \operatorname{tr}^{2}\right) .
\end{aligned}
$$

Similar to the proof of Part $(i)$, let us define

$$
\begin{aligned}
& \Psi=\frac{1}{K} \operatorname{tr} Q \\
& \Phi=\operatorname{tr} Q \frac{H \mathbf{A} H^{\mathrm{H}}}{K} .
\end{aligned}
$$

Putting the results from (368), (369), 377), (379), (381), and (390) together, we conclude that

$$
\begin{aligned}
\mathbb{E}\left[\Phi(1+\Psi) e^{\gamma_{n}^{\mathbf{x}^{n}, H^{n}}}\right]= & -\mathbf{i} t \sqrt{\frac{n}{K}} \frac{\gamma_{1}\left(\sigma^{2}\right)}{1+\delta_{0}\left(\sigma^{2}\right)} \frac{1}{K} \operatorname{tr} \mathbf{A}^{2} \\
& +\mathcal{O}\left(\frac{P_{1}(t)}{\sqrt{K}}+\frac{t P_{2}(t)}{\sqrt{K}} \frac{1}{K} \operatorname{tr} \mathbf{A}^{2}\right)
\end{aligned}
$$

for two polynomials $P_{1}(t)$ and $P_{2}(t)$, where the term $t$ in front of $P_{2}(t)$ arises from the pre-multiplication by at least $\mathbf{i} t$ of the various estimators involved.
We now need to find an alternative representation of the term $\mathbb{E}\left[\Phi \Psi e^{\gamma_{n}^{\mathbf{x}^{n}, H^{n}}}\right]$ in the LHS of the last equation. Following the same arguments as in (356)-(361), and using $\sqrt{K^{-3} \operatorname{tr} \mathbf{A}^{2}} \leq 1 / \sqrt{K}$, we can write

$$
\begin{aligned}
\mathbb{E} & {\left[\Phi \Psi e^{\gamma_{n}^{\mathbf{x}^{n}, H^{n}}}\right] } \\
= & \mathbb{E}[\Psi] \mathbb{E}\left[\Phi e^{\gamma_{n}^{\mathbf{x}^{n}, H^{n}}}\right]+\mathbb{E}\left[\Phi(\Psi-\mathbb{E}[\Psi]) e^{\gamma_{n}^{\mathbf{x}^{n}, H^{n}}}\right] \\
= & \mathbb{E}[\Psi] \mathbb{E}\left[\Phi e^{\gamma_{n}^{\mathbf{x}^{n}, H^{n}}}\right]+\mathbb{E}[\Phi] \mathbb{E}\left[(\Psi-\mathbb{E}[\Psi]) e^{\left.\gamma_{n}^{\mathbf{x}^{n}, H^{n}}\right]}\right. \\
& +\mathcal{O}\left(\frac{1}{\sqrt{K}}\right) \\
= & \mathbb{E}[\Psi] \mathbb{E}\left[\Phi e^{\gamma_{n}^{\mathbf{x}^{n}, H^{n}}}\right]-\mathbb{E}[\Phi] \mathbb{E}[\Psi] \mathbb{E}\left[e^{\gamma_{n}^{\mathbf{x}^{n}, H^{n}}}\right] \\
& +\mathbb{E}[\Phi] \mathbb{E}\left[\Psi e^{\gamma_{n}^{\mathbf{x}^{n}, H^{n}}}\right]+\mathcal{O}\left(\frac{1}{\sqrt{K}}\right) \\
= & \mathbb{E}[\Psi] \mathbb{E}\left[\Phi e^{\gamma_{n}^{\mathbf{x}^{n}, H^{n}}}\right]-\mathbb{E}[\Phi] \mathbb{E}[\Psi] \mathbb{E}\left[e^{\gamma_{n}^{\mathbf{x}^{n}, H^{n}}}\right] \\
& +\mathbb{E}[\Phi] \mathbb{E}\left[\left(\frac{1}{\sigma^{2}} \frac{N}{K}-\frac{1}{\sigma^{2}} \frac{1}{K} \operatorname{tr} Q \frac{H H^{\mathbf{H}}}{K}\right) e^{\gamma_{n}^{\mathbf{x}^{n}, H^{n}}}\right] \\
& +\mathcal{O}\left(\frac{1}{\sqrt{K}}\right) \\
= & \mathbb{E}[\Psi] \mathbb{E}\left[\Phi e^{\gamma_{n}^{\mathbf{x}^{n}, H^{n}}}\right] \\
& +\left(\frac{N}{\sigma^{2}}-K \mathbb{E}[\Psi]\right) \mathbb{E}\left[\frac{\Phi}{K}\right] \mathbb{E}\left[e^{\gamma_{n}^{\mathbf{x}^{n}, H^{n}}}\right] \\
& -\frac{1}{\sigma^{2}} \mathbb{E}\left[\frac{\Phi}{K}\right] \mathbb{E}\left[\operatorname{tr} Q \frac{H H^{\mathrm{H}}}{K} e^{\gamma_{n}^{\mathbf{x}^{n}, H^{n}}}\right]+\mathcal{O}\left(\frac{1}{\sqrt{K}}\right) \\
= & \delta_{0}\left(\sigma^{2}\right) \mathbb{E}\left[\Phi e^{\gamma_{n}^{\mathbf{x}^{n}, H^{n}}}\right]+\mathcal{O}\left(\frac{1}{\sqrt{K}}\right)
\end{aligned}
$$

From the last result and 393), we have

$$
\begin{aligned}
\mathbb{E} & {\left[\Phi(1+\Psi) e^{\gamma_{n}^{\mathbf{x}^{n}, H^{n}}}\right] } \\
= & \left(1+\delta_{0}\left(\sigma^{2}\right)\right) \mathbb{E}\left[\Phi e^{\gamma_{n}^{\mathbf{x}^{n}, H^{n}}}\right]+\mathcal{O}\left(\frac{1}{\sqrt{K}}\right) \\
= & -\mathbf{i} t \sqrt{\frac{n}{K}} \frac{\gamma_{1}\left(\sigma^{2}\right)}{1+\delta_{0}\left(\sigma^{2}\right)} \frac{1}{K} \operatorname{tr} \mathbf{A}^{2} \mathbb{E}\left[e^{\gamma_{n}^{\mathbf{x}^{n}, H^{n}}}\right] \\
& +\mathcal{O}\left(\frac{P_{1}(t)}{\sqrt{K}}+\frac{t P_{2}(t)}{\sqrt{K}} \frac{1}{K} \operatorname{tr} \mathbf{A}^{2}\right)
\end{aligned}
$$

for some polynomials $P_{1}(t)$ and $P_{2}(t)$.

Solving (400) and (401) for $\mathbb{E}\left[\Phi e^{\gamma_{n}^{\mathbf{x}^{n}, H^{n}}}\right]$ yields

$$
\begin{aligned}
\mathbb{E}\left[\Phi e^{\gamma_{n}^{\mathbf{x}^{n}, H^{n}}}\right]= & -\mathbf{i} t \sqrt{\frac{n}{K}} \frac{\gamma_{1}\left(\sigma^{2}\right) \frac{1}{K} \operatorname{tr} \mathbf{A}^{2}}{\left(1+\delta_{0}\left(\sigma^{2}\right)\right)^{2}} \mathbb{E}\left[e^{\gamma_{n}^{\mathbf{x}^{n}, H^{n}}}\right] \\
& +\mathcal{O}\left(\frac{P_{1}(t)}{\sqrt{K}}+\frac{t P_{2}(t)}{\sqrt{K}} \frac{1}{K} \operatorname{tr} \mathbf{A}^{2}\right)
\end{aligned}
$$


This concludes the proof of part (ii).

\section{ACKNOWLEDGMENTS}

We would like to thank the anonymous reviewers for their valuable comments which helped to improve and significantly shorten some of the proofs. In particular, we are indebted to one of the reviewers for providing the proof of Lemma 3 We would like to thank Dr. Laurent Schmalen for the generation of the simulation results for Figure 3 and Prof. Stephan ten Brink for various discussions around the topic of iterative coding systems. We are grateful to Prof. Mérouane Debbah for discussions at the early stage of this work.

\section{REFERENCES}

[1] A. Feinstein, "A new basic theorem of information theory," IRE Trans. Inf. Theory, vol. 4, no. 4, pp. 2-22, Sep. 1954.

[2] C. E. Shannon, "Probability of error for optimal codes in a Gaussian channel," Bell Syst. Tech. J., vol. 38, no. 3, pp. 611-656, May 1959.

[3] E. Biglieri, J. Proakis, and S. Shamai, "Fading channels: Informationtheoretic and communications aspects," IEEE Trans. Inf. Theory, vol. 44 no. 6, pp. 2619-2692, Oct. 1998.

[4] C. Shannon, "Certain results in coding theory for noisy channels," Information and control, vol. 1, no. 1, pp. 6-25, Sep. 1957.

[5] R. Gallager, "A simple derivation of the coding theorem and some applications," IEEE Trans. Inf. Theory, vol. 11, no. 1, pp. 3-18, Jan. 1965.

[6] H. Shin and M. Z. Win, "Gallager's exponent for MIMO channels: A reliability-rate tradeoff," IEEE Trans. Commun., vol. 57, no. 4, pp. 972 985, Apr. 2009.

[7] V. Strassen, "Asymptotische Abschätzugen in Shannon's Informationstheorie," in Trans. Third Prague Conf. Inf. Theory, Statist. Decision Functions, Random Processes, Czechoslovak Academy of Sciences, Prague, Czech Repulic, 1962, pp. 689-723.

[8] M. Hayashi, "Information spectrum approach to second-order coding rate in channel coding," IEEE Trans. Inf. Theory, vol. 55, no. 11, pp. 4947-4966, Nov. 2009.

[9] Y. Polyanskiy, H. V. Poor, and S. Verdú, "Channel coding rate in the finite blocklength regime," IEEE Trans. Inf. Theory, vol. 56, no. 5, pp. 2307-2359, May 2010.

[10] Y. Polyanskiy and S. Verdú, "Scalar coherent fading channel: Dispersion analysis," in Proc. IEEE Int. Symp. Inf. Theory (ISIT), Saint Petersburg, Russia, Aug. 2011, pp. 2959-2963.

[11] W. Yang, G. Durisi, T. Koch, and Y. Polyanskiy, "Diversity versus channel knowledge at finite block-length," in Proc. IEEE Information Theory Workshop (ITW), Lausanne, Switzerland, Sep. 2012, pp. 572 576.

[12] S. Verdú and T. S. Han, "A general formula for channel capacity," IEEE Trans. Inf. Theory, vol. 40, no. 4, pp. 1147-1157, Jul. 1994.

[13] J. Wolfowitz, "Notes on a general strong converse," Information and Control, vol. 12, no. 1, pp. 1-4, Jan. 1968.

[14] T. S. Han and S. Verdú, "Approximation theory of output statistics," IEEE Trans. Inf. Theory, vol. 39, no. 3, pp. 752-772, May 1993.

[15] W. Yang, G. Durisi, T. Koch, and Y. Polyanskiy, "Quasi-static SIMO fading channels at finite blocklength," in Proc. IEEE Int. Symp. Inf. Theory (ISIT), Istanbul, Turkey, Jul. 2013.

[16] - "Quasi-static multiple-antenna fading channels at finite blocklength," IEEE Trans. Inf. Theory, vol. 60, no. 7, pp. 4232-4265, Jun. 2014.

[17] L. A. Pastur and M. Shcherbina, Eigenvalue distribution of large random matrices. Providence, RI, USA: American Mathematical Society, Mathematical Surveys and Monographs, 2011, vol. 171.

[18] P. Billingsley, Probability and Measure, 3rd ed. John Wiley \& Sons, Inc., 1995.

[19] T. S. Han, Information-Spectrum Methods in Information Theory. Springer-Verlag, 2003.

[20] S. Verdú and S. Shamai, "Spectral efficiency of CDMA with random spreading," IEEE Trans. Inf. Theory, vol. 45, no. 2, pp. 622-640, Mar. 1999.

[21] W. Hachem, O. Khorunzhiy, P. Loubaton, J. Najim, and L. Pastur, "A new approach for mutual information analysis of large dimensional multi-antenna channels," IEEE Trans. Inf. Theory, vol. 54, no. 9, pp. 3987-4004, Sep. 2008.
[22] W. Hachem, P. Loubaton, and J. Najim, "A CLT for information theoretic statistics of Gram random matrices with a given variance profile," The Annals of Probability, vol. 18, no. 6, pp. 2071-2130, Dec. 2008.

[23] V. A. Marčenko and L. A. Pastur, "Distributions of eigenvalues for some sets of random matrices," Math USSR-Sbornik, vol. 1, no. 4, pp. 457483, April 1967.

[24] Z. D. Bai and J. W. Silverstein, "CLT for linear spectral statistics of large-dimensional sample covariance matrices," The Annals of Probability, vol. 32, no. 1A, pp. 553-605, 2004.

[25] IEEE, "IEEE, 802.16, Air Interface for Broadband Wireless Access Systems," 2009.

[26] I. Csiszár and J. Körner, Information theory: coding theorems for discrete memoryless systems. Academic, New York, 1981.

[27] J. Wendel, "Note on the gamma function," American Mathematical Monthly, pp. 563-564, 1948.

[28] R. Couillet and M. Debbah, Random matrix methods for wireless communications, 1st ed. New York, NY, USA: Cambridge University Press, 2011

Jakob Hoydis (S'08-M'12) received the diploma degree (Dipl.-Ing.) in electrical engineering and information technology from RWTH Aachen University, Germany, and the Ph.D. degree from Supélec, Gif-sur-Yvette, France, in 2008 and 2012, respectively. From 2012-2013, he worked for Bell Laboratories, Alcatel-Lucent, Stuttgart, Germany, on next-generation mobile communication systems. From 2014-2015, he was co-founder and technical director of Spraed, Orsay, France. In September 2015, he joined Bell Labs France in the "Software-Defined Wireless Networks"-Department. $\mathrm{He}$ is recipient of the 2012 Publication Prize of the Supélec Foundation, the 2013 VDE ITG Förderpreis and the 2015 Leonard G. Abraham Prize of the IEEE Communications Society. He received the WCNC'2014 best paper award and has been nominated as an Exemplary Reviewer 2012 for the IEEE Communication letters. His research interests are in the areas of cloud computing, SDR, large random matrix theory, information theory, signal processing and their applications to wireless communications.

Romain Couillet (S'07-M'11) received his MSc in Mobile Communications at the Eurecom Institute and his MSc in Communication Systems in Telecom ParisTech, France in 2007. From 2007 to 2010, he worked with STEricsson as an Algorithm Development Engineer on the Long Term Evolution Advanced project, where he prepared his $\mathrm{PhD}$ with Supelec, France, which he graduated in November 2010. He is currently an assistant professor in the Telecommunication department of CentraleSupélec, France. His research topics are in random matrix theory applied to wireless communications, signal processing, and statistics. In 2015, he received the HDR title from University ParisSud. He is the recipient of the 2013 CNRS Bronze Medal in the section "science of information and its interactions", of the 2013 IEEE ComSoc Outstanding Young Researcher Award (EMEA Region), of the 2011 EEA/GdR ISIS/GRETSI best PhD thesis award, and of the Valuetools 2008 best student paper award.

Pablo Piantanida (S'04-M'08) received both B.Sc. in Electrical Engineering (with honors) and B.Sc. in Mathematics, and M.Sc degrees from the University of Buenos Aires (Argentina) in 2003, and the Ph.D. from Université Paris-Sud (Orsay, France) in 2007. Since October 2007 he has joined the Laboratoire des Signaux et Systèmes (L2S), CentraleSupélec-CNRS, as an Assistant Professor in Network Information Theory. His research interests include multiterminal information theory, Shannon theory, machine learning, cooperative communications, physical-layer security and distributed source coding. 